\title{
Supporting Information for: Catalytic Double Carbonylation of Epoxides to Succinic Anhydrides: Catalyst Discovery, Reaction Scope and Mechanism
}

\author{
John M. Rowley, Emil B. Lobkovsky, and Geoffrey W. Coates* \\ Department of Chemistry and Chemical Biology, Baker Laboratory, \\ Cornell University, Ithaca, New York 14853-1301
}

Catalyst Screening for Double Carbonylation. Once we found that double carbonylation with 1 was most active in 1,4-dioxane, we returned to investigate the Lewis acid component of the catalyst. In Figure S1, a number of new and previously reported variations of the general form $\left[(\text { ligand }) \mathrm{M}(\mathrm{THF})_{2}\right]^{+}\left[\mathrm{Co}(\mathrm{CO})_{4}\right]^{-}$(denoted as '(ligand)M') are compared under the optimized reaction conditions for the double carbonylation of 1,2-epoxybutane in 1,4-dioxane. Under these conditions $\mathrm{Cp}_{2} \mathrm{Ti}(\mathrm{Cp}=$ cyclopentadienyl) only converted $6 \%$ of the epoxide to lactone, and made no anhydride. The only catalyst previously reported for lactone carbonylation, (salph)Al

Figure S1. Epoxide double carbonylation optimization: screening catalyst Lewis acid, ligand and metal. ${ }^{a}$
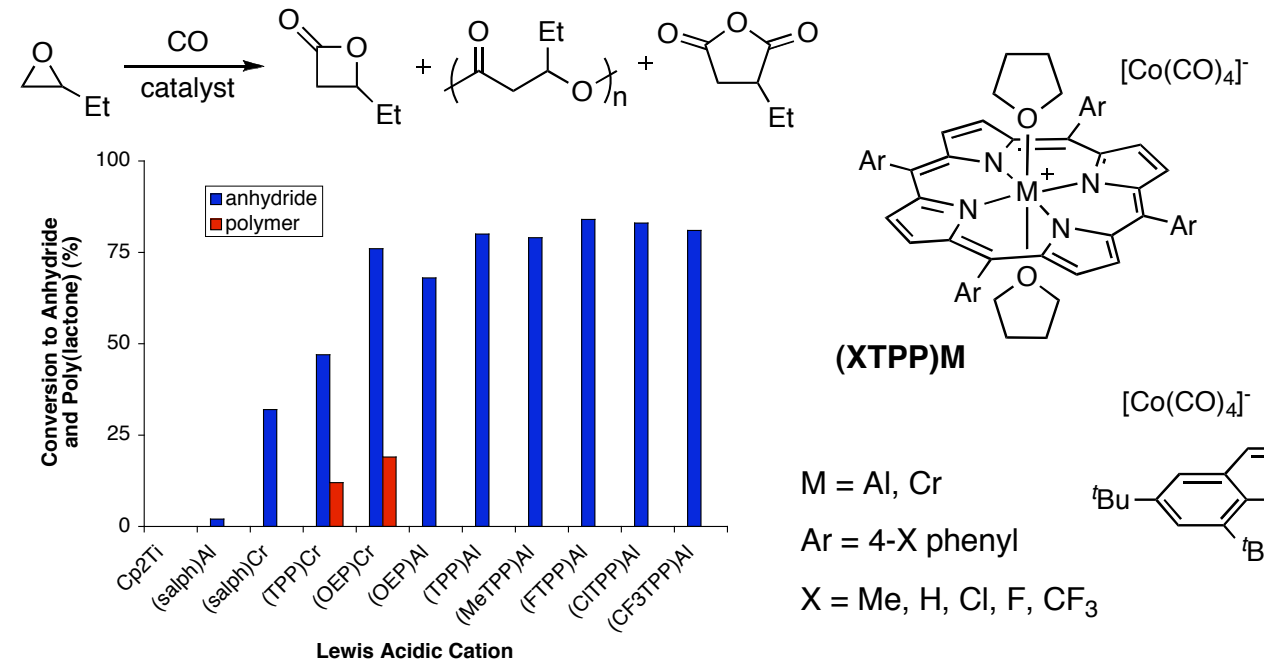

$$
\begin{aligned}
& \mathrm{M}=\mathrm{Al}, \mathrm{Cr} \\
& \mathrm{Ar}=4-\mathrm{X} \text { phenyl } \\
& \mathrm{X}=\mathrm{Me}, \mathrm{H}, \mathrm{Cl}, \mathrm{F}, \mathrm{CF}_{3}
\end{aligned}
$$
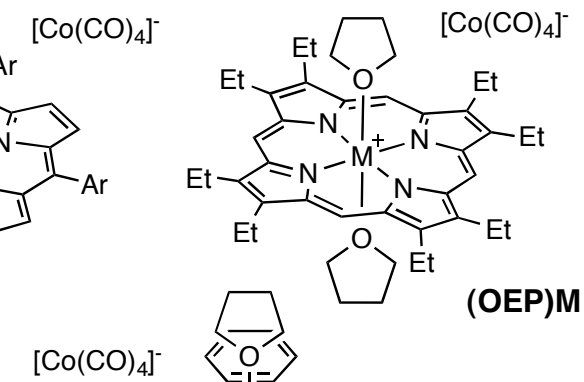

\footnotetext{
${ }^{a}$ Reaction conditions: $2 \mathrm{mmol}$ 1,2-epoxybutane in $1 \mathrm{~mL}$ dioxane, 0.2 mol\% catalyst, $850 \mathrm{psi} \mathrm{CO}, 2 \mathrm{~h}, 90{ }^{\circ} \mathrm{C}$. Product distribution determined by ${ }^{1} \mathrm{H}$ NMR spectrum of crude reaction mixture. All catalysts, except $\mathrm{Cp}_{2} \mathrm{Ti}$, completely converted epoxide to lactone (not shown). Subsequent conversion of lactone to anhydride and polymer for other catalysts is shown.
} 
(2), was actually quite slow for double carbonylation. The chromium analog, (salph)Cr, was much faster, as were (TPP)Cr and (OEP)Cr (3), though the porphyrin $\mathrm{Cr}$ complexes gave poly(lactone) as a significant byproduct. Relative to the tetraphenylporphyrin (TPP) ligands, the more electron-donating octaethylporphyrin (OEP) ligand diminished the activity of the Al complexes. Tetraphenylporphyrin aluminum cations appear to be the most effective for clean, catalytic, one-pot double carbonylation of epoxides to succinic anhydrides. A series of catalysts with substituted porphyrin phenyl rings were synthesized $(\mathrm{XTPP}=$ meso-tetra $(4-\mathrm{X}-$ phenyl)porphyrinato), but this substitution had little effect on the activity of the catalysts (Figure S1).

Calibration Curves in 1,4-Dioxane. In the IR spectrum of $\beta$-butyrolactone (BBL) the magnitude of the absorbance at $1827 \mathrm{~cm}^{-1}$ depended linearly on concentration from 0 to $1.0 \mathrm{M}$. For methylsuccinic anhydride (MSA), there was also a linear relationship between the magnitude of the IR absorbance $\left(1787 \mathrm{~cm}^{-1}\right)$ and concentration, from 0 to $0.2 \mathrm{M}$. However, above $\sim 0.4 \mathrm{M}$ there was significant deviation from linearity. Using a polynomial best fit curve, it was possible to convert absorbance to concentration for the entire range 0 to $1.0 \mathrm{M}$; however, initial rates were

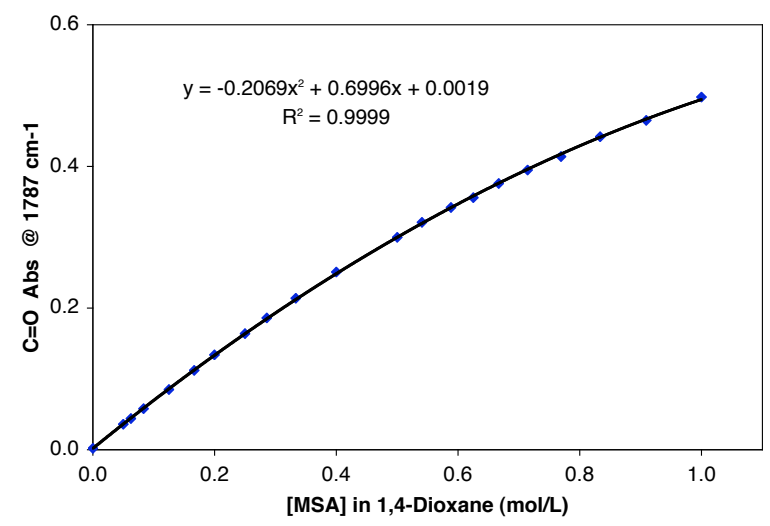

Figure S2. Calibration curve of MSA in 1,4-dioxane. 
calculated from the first 5 to $20 \%$ of the reaction when absorbance still varied linearly with respect to $[\mathrm{MSA}]$. The calibration curve was prepared from IR absorbance measurements of solutions of known MSA concentration in the modified Parr reactor used to run in situ IR experiments (Figure S2).

Effect of CO Pressure on Initial Rates. For the double carbonylation of propylene oxide (PO), the order in $\mathrm{CO}$ was determined by varying the pressure from 100 to 1000 psi (Figure S3). Initial rates of $\mathrm{BBL}$ and MSA formation were measured from the same reaction. The rates were essentially unchanged over a wide range of $\mathrm{CO}$ pressure; only slight variations due to experimental error were observed. Data were not collected for epoxide carbonylation at 100 psi due to the formation of a small amount of ketone byproduct.

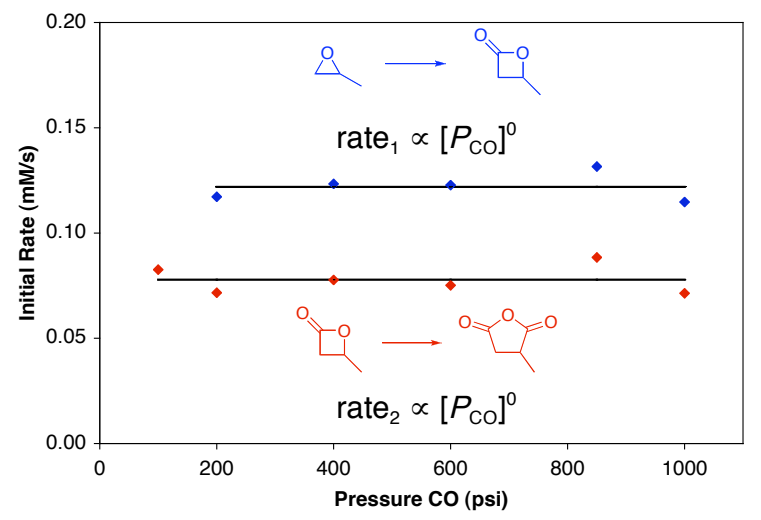

Figure S3. Dependence of the initial rates of epoxide and lactone carbonylation on $P_{\mathrm{CO}}$. Reactions performed in 1,4-dioxane and monitored with in situ IR spectroscopy $\left(v_{\mathrm{C}=\mathrm{O}} \mathrm{BBL}=1827 \mathrm{~cm}^{-1}\right.$ and $v_{\mathrm{C}=\mathrm{O}}$ MSA $\left.1787 \mathrm{~cm}^{-1}\right) .[\mathrm{PO}]_{0}$ or $[\mathrm{BBL}]_{0}=1.0 \mathrm{M},[\mathbf{1}]=2.0 \mathrm{mM}, \mathrm{T}$ $=40{ }^{\circ} \mathrm{C}$.

Inverse Solvent Dependence. When the carbonylation of BBL was conducted in mixtures of THF and DMTHF, the addition of a small amount of THF resulted in a drastic reduction of the initial rate of carbonylation. A plot of initial rate versus the inverse concentration of THF in DMTHF demonstrates a linear relationship, indicating that the rate of lactone carbonylation 
depends inversely on the concentration of THF (Figure S4). The linear fit of these data points was used to calculate the trendline for initial rates used in Figure 6:

$$
\text { initial rate }=21.3 /[\mathrm{THF}]-1.25
$$

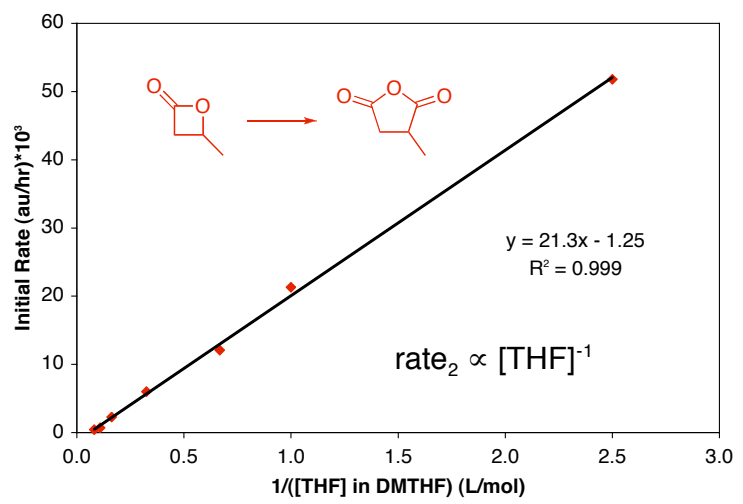

Figure S4. Effect of $[\mathrm{THF}]$ on the rate of carbonylation of $\mathrm{PO}$ and $\mathrm{BBL}$ in mixtures of THF/DMTHF. $1 /[\mathrm{THF}]=0.08 \mathrm{M}^{-1}$ corresponds to pure THF. Reactions monitored with in situ IR spectroscopy $\left(v_{\mathrm{C}=\mathrm{O}} \mathrm{MSA}=1787 \mathrm{~cm}^{-1}\right) .[\mathrm{BBL}]_{0}=1.0$ $\mathrm{M},[\mathbf{1}]=2.0 \mathrm{mM}, P_{\mathrm{CO}}=850 \mathrm{psi}, T=40^{\circ} \mathrm{C}$.

Simulation. The theoretical concentrations of lactone and anhydride, as predicted by our proposed mechanism and measured rate constants, were calculated as a function of time. In order to simplify our calculations, we assumed that exchange between coordinated solvent, epoxide,

ring-opening of $\mathrm{PO}$

$$
\mathrm{PO}+1 \stackrel{\mathrm{k}_{1}}{\longrightarrow} \mathrm{A} \quad \mathrm{d}[\mathrm{PO}] / \mathrm{dt}=-\mathrm{k}_{1}[\mathrm{PO}][1]
$$

ring-closing to $\mathrm{BBL}$

$$
\mathrm{A} \stackrel{\mathrm{k}_{2}}{\longrightarrow} \mathrm{BBL}+1
$$

ring-opening of $\mathrm{BBL}$

$$
\mathrm{BBL}+\mathbf{1} \stackrel{\mathrm{k}_{3}}{\longrightarrow} \mathrm{B}
$$

$$
\mathrm{d}[\mathrm{BB} L] / \mathrm{dt}=\mathrm{k}_{2}[\mathrm{~A}]-\mathrm{k}_{3}[\mathrm{~B}][1]
$$$$
\mathrm{d}[\mathrm{MSA}] / \mathrm{dt}=\mathrm{k}_{4}[\mathrm{~B}]
$$$$
d[1] / d t=-k_{1}[P O][1]+k_{2}[A]-k_{3}[B B L][1]+k_{4}[B]
$$$$
d[A] / d t=k_{1}[P O][1]-k_{2}[A]
$$$$
\mathrm{d}[\mathrm{B}] / \mathrm{dt}=\mathrm{k}_{3}[\mathrm{BBL}][1]-\mathrm{k}_{4}[\mathrm{~B}]
$$

ring-closing to MSA

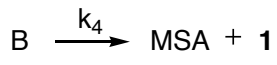

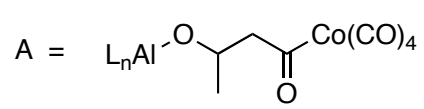

$$
\begin{array}{ll}
{[\mathrm{PO}]_{0}=1.00 \mathrm{M}} & \mathrm{k}_{1}=60 \mathrm{mM}^{-1} \mathrm{~s}^{-1} \\
{[\mathrm{BBL}]_{0}=0} & \mathrm{k}_{2}=0.060 \mathrm{~s}^{-1} \\
{[\mathrm{MSA}]_{0}=0} & \\
{[1]_{0}=0.004 \mathrm{M}} & \mathrm{k}_{3}=0.039 \mathrm{mM}^{-1} \mathrm{~s} \\
{[\mathrm{~A}]_{0}=0} & \\
{[B]_{0}=0} & \mathrm{k}_{4}=3.9 \mathrm{~s}^{-1}
\end{array}
$$

Figure S5. Elemental reactions, differential equations, rate constants, and initial concentrations used to calculate theoretical concentrations of BBL and MSA. 
lactone, and anhydride was fast and reversible on the time scale of carbonylation. We also treated the uptake and migratory insertion of $\mathrm{CO}$ as very rapid with an equilibrium lying far to the side of cobalt acyl under pressures of 850 psi CO. ${ }^{1,2}$ The concentration of solvent or Lewis base remains constant throughout the reaction and was included in the rate constant. Finally, ring closing of epoxide to form lactone and ring opening of coordinated lactone were designated as the rate-determining steps. With these assumptions it was possible to derive a series of differential equations for the concentration of the important species (Figure S5). From our kinetic studies we extracted rate constants for the rate-determining steps $\left(k_{2}\right.$ and $k_{3}$, from Figure 4$)$, and estimated constants for the faster steps that were significantly greater. Using typical experimental conditions for the double carbonylation of PO as initial concentrations, we solved for the concentration of BBL and MSA as a function of time using the program Scientist ${ }^{\circledR}$, from MicroMath Scientific Software Inc.

Experimental. All materials and carbonylation procedures were the same as reported in the full text. $\left[\mathrm{Cp}_{2} \mathrm{Ti}(\mathrm{THF})_{2}\right]^{+}\left[\mathrm{Co}(\mathrm{CO})_{4}\right]^{-3}, \quad\left[(\mathrm{salph}) \mathrm{Al}(\mathrm{THF})_{2}\right]^{+}\left[\mathrm{Co}(\mathrm{CO})_{4}\right]^{-4}, \quad\left[(\mathrm{salph}) \mathrm{Cr}(\mathrm{THF})_{2}\right]^{+}$ $\left[\mathrm{Co}(\mathrm{CO})_{4}\right]^{-5},\left[(\mathrm{TPP}) \mathrm{Cr}(\mathrm{THF})_{2}\right]^{+}\left[\mathrm{Co}(\mathrm{CO})_{4}\right]^{-6},{ }^{-}$and $\left[(\mathrm{OEP}) \mathrm{Cr}(\mathrm{THF})_{2}\right]^{+}\left[\mathrm{Co}(\mathrm{CO})_{4}\right]^{-7}$, were synthesized as previously reported.

\section{Bis(tetrahydrofuran)-octaethylporphyrinato aluminum tetracarbonyl cobaltate,} $\left[(\mathbf{O E P}) \mathbf{A l}(\mathbf{T H F})_{2}\right]^{+}\left[\mathrm{Co}(\mathrm{CO})_{4}\right]^{-}$. The synthetic procedure was identical to that for $\mathbf{1}$, but used the commercially available octaethylporphyrin ligand. Starting from OEP ligand (175 mg, 327 $\mu \mathrm{mol})$ the complex was synthesized as dark red crystals (195 mg, 68\%). ${ }^{1} \mathrm{H}$ NMR (300 MHz, $\left.\mathrm{C}_{6} \mathrm{D}_{6}, \delta\right): 10.56(\mathrm{~s}, 4 \mathrm{H}), 4.12(\mathrm{q}, 16 \mathrm{H}), 1.92(\mathrm{t}, 24 \mathrm{H}),-1.42$ (br s, 8H), -2.64 (br s, 8H); IR (Nujol, $\mathrm{NaCl}$ ) $v_{\mathrm{C}=\mathrm{O}}=1885 \mathrm{~cm}^{-1}$. Structure confirmed by X-ray crystallography, and is isostructural with $\left[(\mathrm{OEP}) \mathrm{Cr}(\mathrm{THF})_{2}\right]^{+}\left[\mathrm{Co}(\mathrm{CO})_{4}\right]^{-}$. 
tetracarbonyl cobaltate, $\left[(\mathrm{MeTPP}) \mathrm{Al}(\mathrm{THF})_{2}\right]^{+}\left[\mathrm{Co}(\mathbf{C O})_{4}\right]^{-}$. The synthetic procedure was identical to that for $\mathbf{1}$, but employed 4-methylbenzaldehyde in porphyrin ligand synthesis. ${ }^{8}$ From meso-tetra(4-methylphenyl)porphyrin $(671 \mathrm{mg}, 1.00 \mathrm{mmol})$ the complex was isolated as dark purple crystalline solid (680 mg, 67\%). ${ }^{1} \mathrm{H}$ NMR (300 MHz, $\left.\mathrm{C}_{6} \mathrm{D}_{6}, \delta\right): 9.19(\mathrm{~s}, 8 \mathrm{H}), 8.15$ (m, 8H), $7.40(\mathrm{~m}, 8 \mathrm{H}), 2.39(\mathrm{~s}, 12 \mathrm{H}) ; \mathrm{IR}$ (Nujol, $\mathrm{NaCl}) v_{\mathrm{C}=\mathrm{O}}=1880 \mathrm{~cm}^{-1}$.

Bis(tetrahydrofuran)-meso-tetraphenylporphyrinato aluminum tetracarbonyl cobaltate, $\left[(\mathbf{T P P}) \mathbf{A l}(\mathbf{T H F})_{2}\right]^{+}\left[\mathbf{C o}(\mathbf{C O})_{4}\right]^{-}$. The synthetic procedure was identical to that for $\mathbf{1}$, but employed benzaldehyde in porphyrin ligand synthesis. ${ }^{8}$ From tetraphenylporphyrin $(1.45 \mathrm{~g}, 2.37$ mmol) the complex was isolated as dark purple crystalline plates $(2.16 \mathrm{~g}, 95 \%) .{ }^{1} \mathrm{H}$ NMR (300 $\left.\mathrm{MHz}, \mathrm{C}_{6} \mathrm{D}_{6}, \delta\right): 9.08(\mathrm{~s}, 8 \mathrm{H}), 8.17$ (m, 8H), 7.52 (m, 12H), 3.38 (br s, 8H), 1.18 (br s, 8H); IR (Nujol, $\mathrm{NaCl}$ ) $v_{\mathrm{C}=\mathrm{O}}=1875 \mathrm{~cm}^{-1}$.

Bis(tetrahydrofuran)-meso-tetra(4-fluorophenyl)porphyrinato aluminum tetracarbonyl cobaltate, $\left[(\mathbf{F T P P}) \mathbf{A l}(\mathbf{T H F})_{2}\right]^{+}\left[\mathbf{C o}(\mathbf{C O})_{4}\right]^{-}$. The synthetic procedure was identical to that for $\mathbf{1}$, but employed 4-fluorobenzaldehyde in porphyrin ligand synthesis. ${ }^{8}$ From meso-tetra(4fluorophenyl)porphyrin $(410 \mathrm{mg}, 0.597 \mathrm{mmol})$ the complex was isolated as dark purple crystals (340 mg, 66\%). ${ }^{1} \mathrm{H}$ NMR (300 MHz, THF- $\left.d_{8}, \delta\right): 9.21(\mathrm{~s}, 8 \mathrm{H}), 8.21(\mathrm{~m}, 8 \mathrm{H}), 7.42(\mathrm{~m}, 8 \mathrm{H}), 3.58$ $(\mathrm{m}, 8 \mathrm{H}), 1.74(\mathrm{~m}, 8 \mathrm{H}) ; \mathrm{IR}$ (Nujol, $\mathrm{NaCl}) v_{\mathrm{C}=\mathrm{O}}=1875 \mathrm{~cm}^{-1}$.

Bis(tetrahydrofuran)-meso-tetra(4-trifluoromethylphenyl)porphyrinato aluminum tetracarbonyl cobaltate, $\left[\left(\mathbf{C F}_{3} \mathbf{T P P}\right) \mathbf{A l}(\mathbf{T H F})_{2}\right]^{+}\left[\mathrm{Co}(\mathbf{C O})_{4}\right]^{-}$. The synthetic procedure was identical to that for $\mathbf{1}$, but employed 4-trimethylfluorobenzaldehyde in porphyrin ligand synthesis. ${ }^{8}$ From meso-tetra(4-trifluoromethylphenyl)porphyrin $(242 \mathrm{mg}, 0.273 \mathrm{mmol})$ the 
complex was isolated as dark purple crystalline solid $(258 \mathrm{mg}, 81 \%)$. IR $(\mathrm{Nujol}, \mathrm{NaCl}) v_{\mathrm{C}=\mathrm{O}}=$ $1876 \mathrm{~cm}^{-1}$.

\section{References}

(1) Church, T. L.; Getzler, Y. D. Y. L.; Coates, G. W. J. Am. Chem. Soc. 2006, 128, 1012510133.

(2) Galamb, V.; Palyi, G. Coord. Chem. Rev. 1984, 59, 203-238.

(3) Mahadevan, V.; Getzler, Y. D. Y. L.; Coates, G. W. Angew. Chem. Int. Ed. 2002, 41, 27812784.

(4) Getzler, Y. D. Y. L.; Mahadevan, V.; Lobkovsky, E. B.; Coates, G. W. J. Am. Chem. Soc. 2002, 124, 1174-1175.

(5) Kramer, J. W.; Lobkovsky, E. B.; Coates, G. W. Org. Lett. 2006, 8, 3709-3712.

(6) Schmidt, J. A. R.; Mahadevan, V.; Getzler, Y. D. Y. L.; Coates, G. W. Org. Lett. 2004, 6, 373376.

(7) Schmidt, J. A. R.; Lobkovsky, E. B.; Coates, G. W. J. Am. Chem. Soc. 2005, 127, 1142611435.

(8) Adler, A. D.; Longo, F. R.; Finarelli, J. D.; Goldmacher, J.; Assour, J.; Korsakoff, L. J. Org. Chem. 1967, 32, 476. 
Figure S6. ${ }^{1} \mathrm{H}$ NMR spectrum of $\mathbf{1 1}, n$-butylsuccinic anhydride $\mathrm{CDCl}_{3}, 300 \mathrm{MHz}$ (Table 2, entry 4).

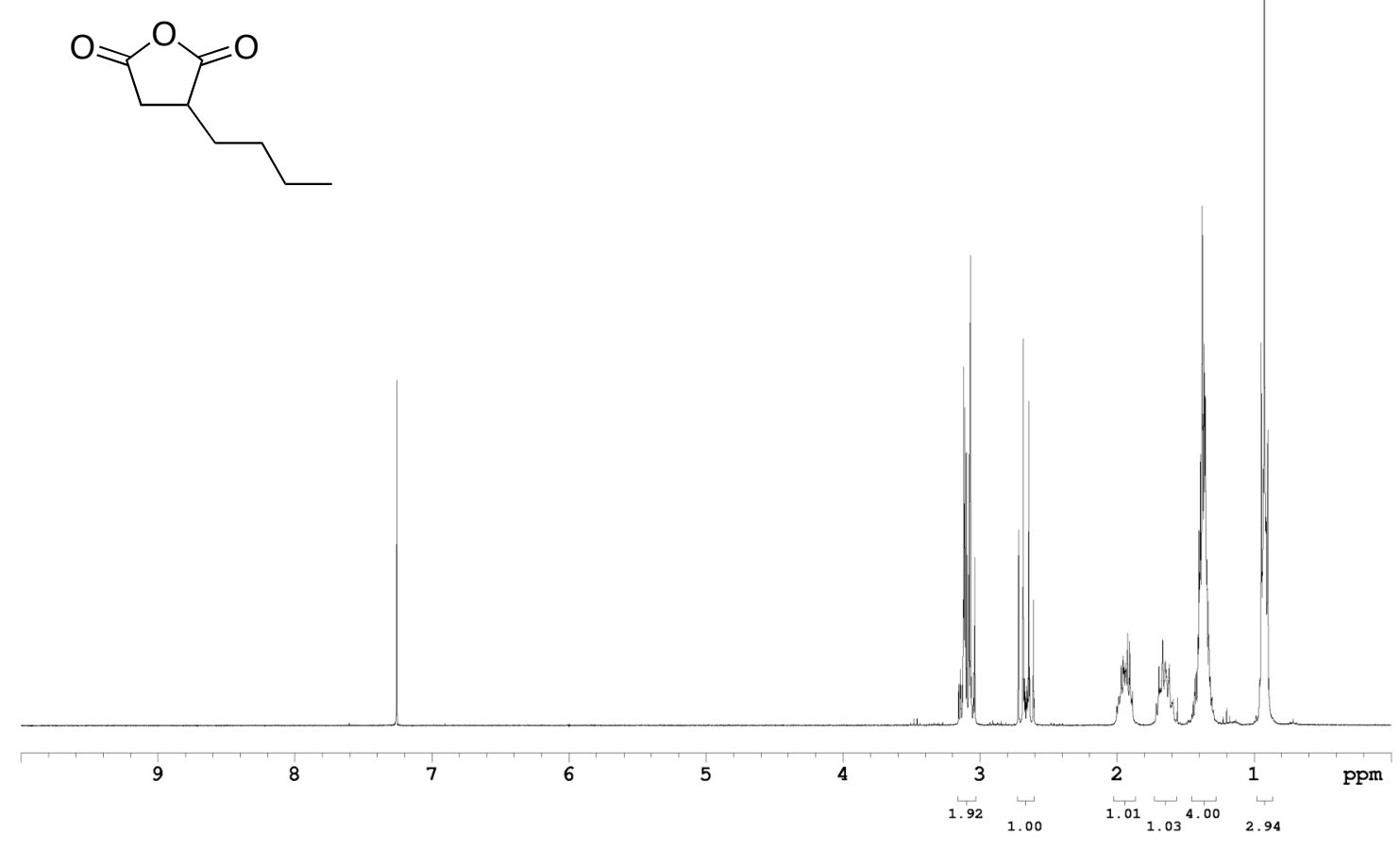

Figure $57 .{ }^{13} \mathrm{C}$ NMR spectrum of $\mathbf{1 1}, n$-butylsuccinic anhydride $\mathrm{CDCl}_{3}, 75 \mathrm{MHz}$ (Table 2, entry 4).

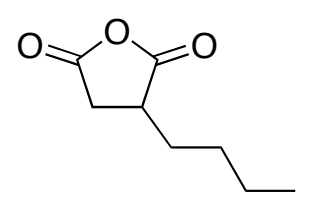

J

$\begin{array}{llll}180 & 160 & 140 & 120\end{array}$

100

80

60

40

20

ppm 
Figure S8. ${ }^{1} \mathrm{H}$ NMR spectrum of 15 , cyclohexylsuccinic anhydride $\mathrm{CDCl}_{3}, 300 \mathrm{MHz}$ (Table 2, entry 6).

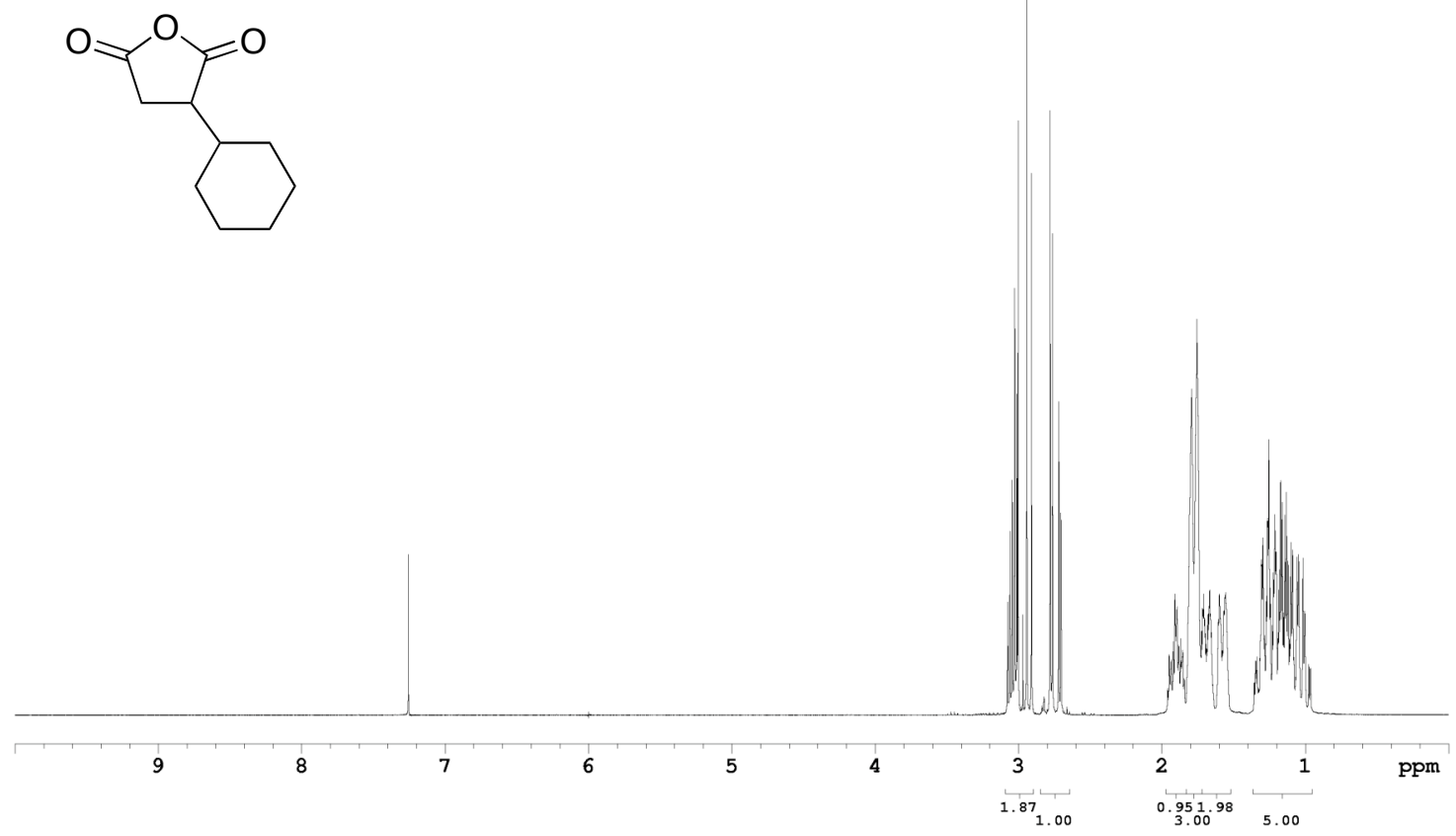

Figure S9. ${ }^{13} \mathrm{C}$ NMR spectrum of 15 , cyclohexylsuccinic anhydride $\mathrm{CDCl}_{3}, 75 \mathrm{MHz}$ (Table 2, entry 6).

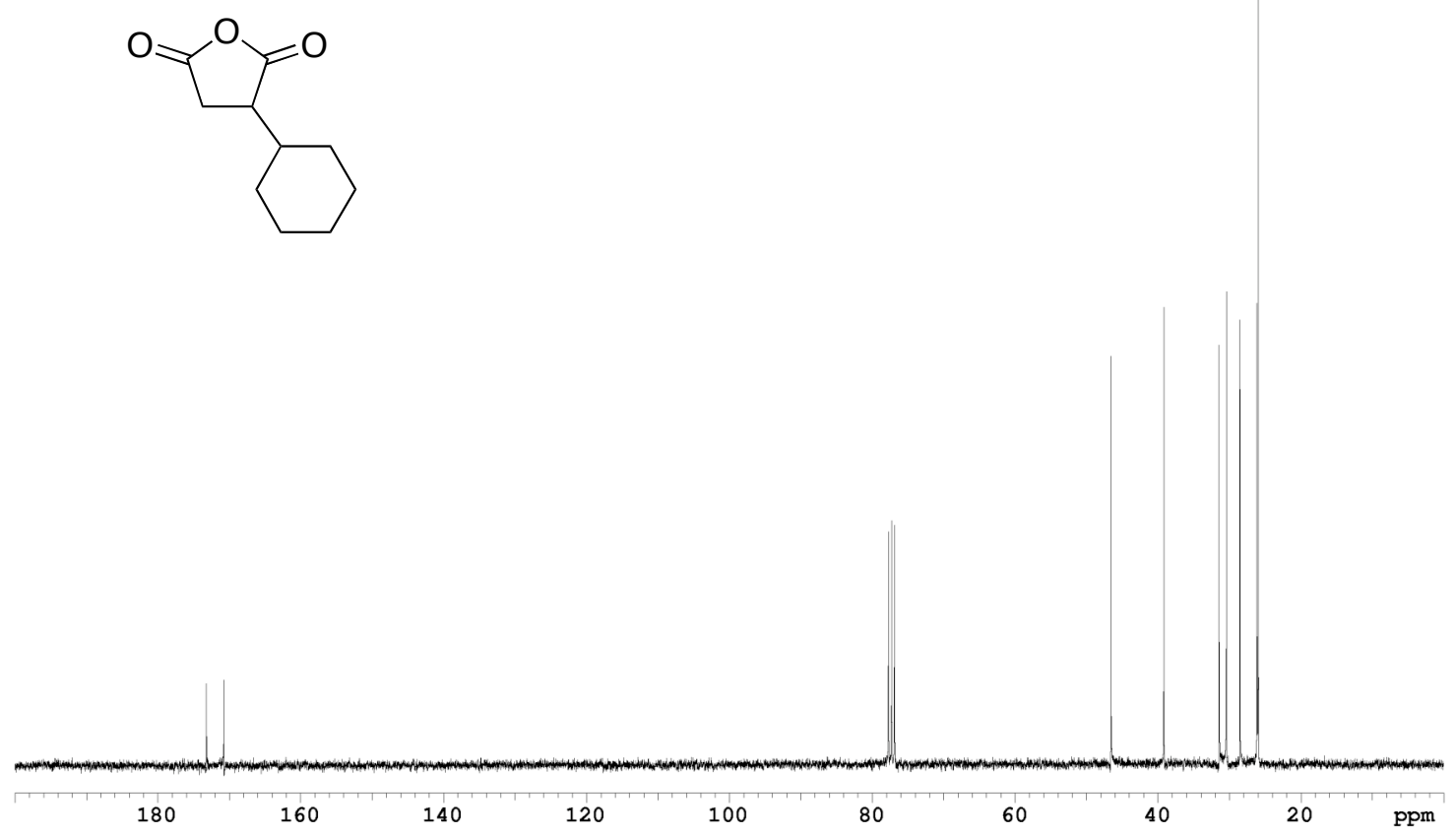


Figure S10. ${ }^{1} \mathrm{H}$ NMR spectrum of 21, benzyloxymethylsuccinic anhydride $\mathrm{CDCl}_{3}, 300 \mathrm{MHz}$ (Table 2, entry 9).

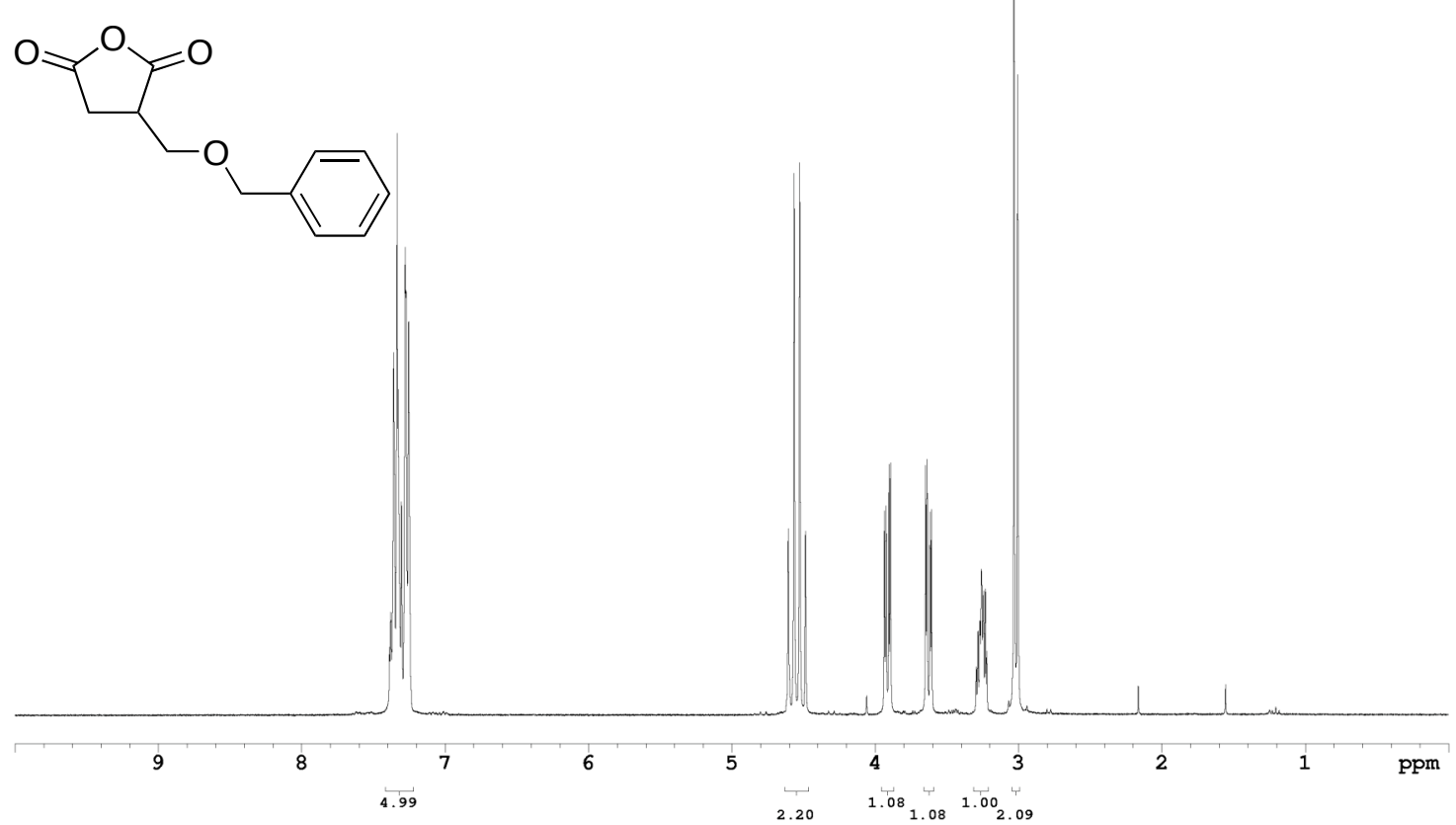

Figure S11. ${ }^{13} \mathrm{C}$ NMR spectrum of 21, benzyloxymethylsuccinic anhydride $\mathrm{CDCl}_{3}, 75 \mathrm{MHz}$ (Table 2, entry 9).

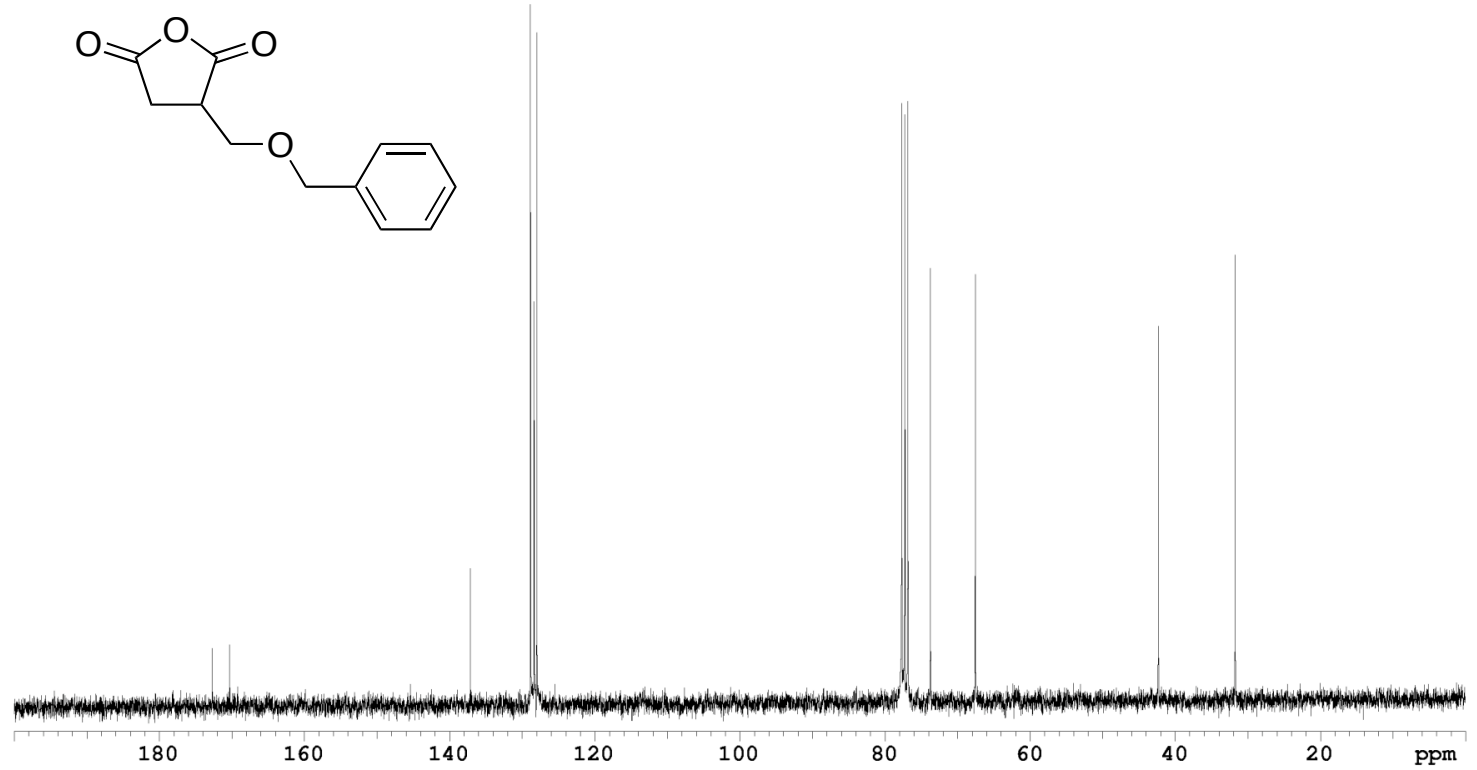


Figure S12. ${ }^{1} \mathrm{H}$ NMR spectrum of 23, 9-hydroxynonylsuccinic anhydride $\mathrm{CDCl}_{3}, 300 \mathrm{MHz}$ (Table 2, entry 10).

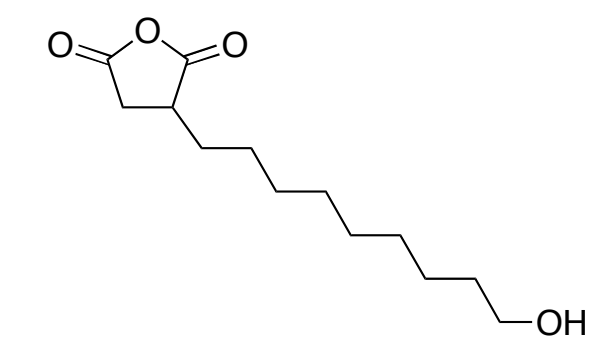

Figure S13. ${ }^{13} \mathrm{C}$ NMR spectrum of 23 , 9-hydroxynonylsuccinic anhydride $\mathrm{CDCl}_{3}, 75 \mathrm{MHz}$ (Table 2, entry 10).

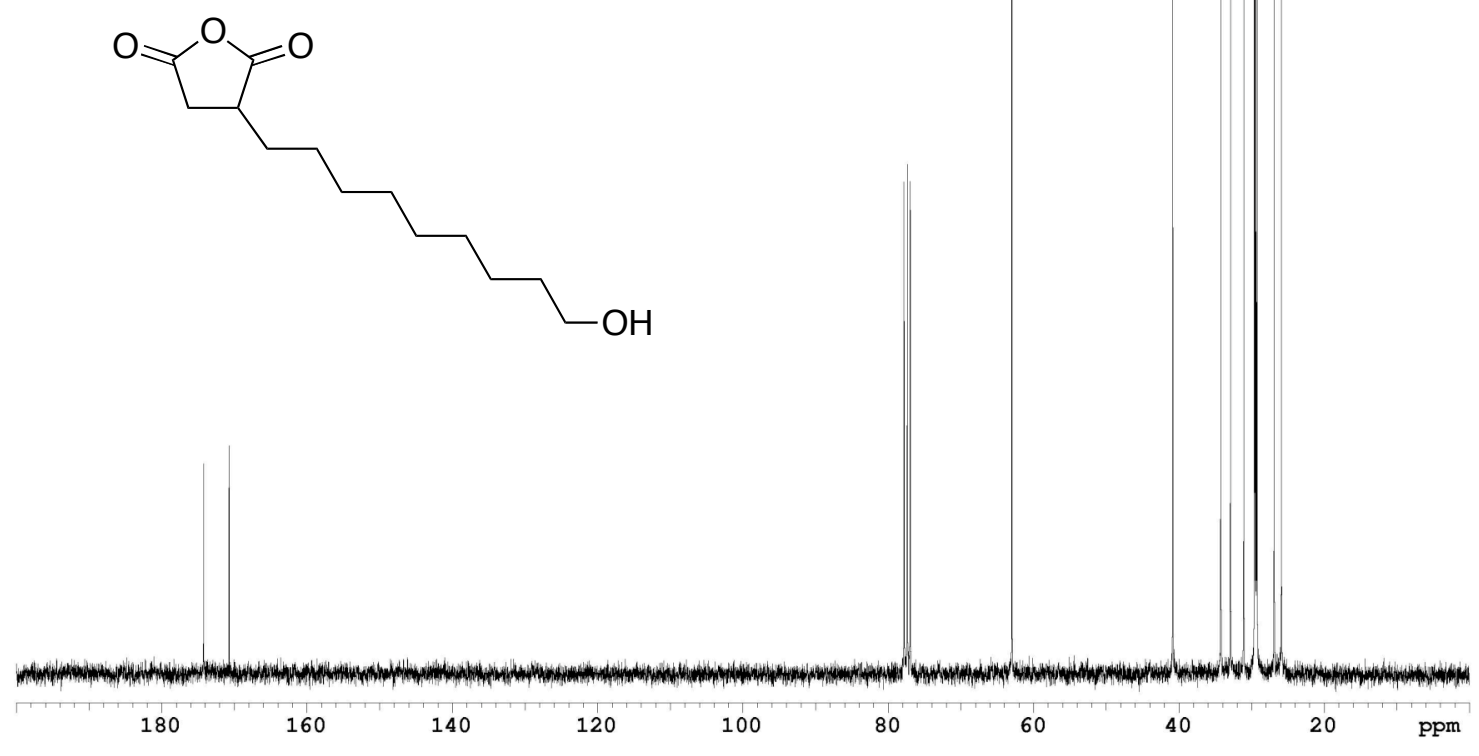


Figure S14. ${ }^{1} \mathrm{H}$ NMR spectrum of 25, 3-butyroxypropylsuccinic anhydride $\mathrm{CDCl}_{3}, 300 \mathrm{MHz}$ (Table 2, entry 11).

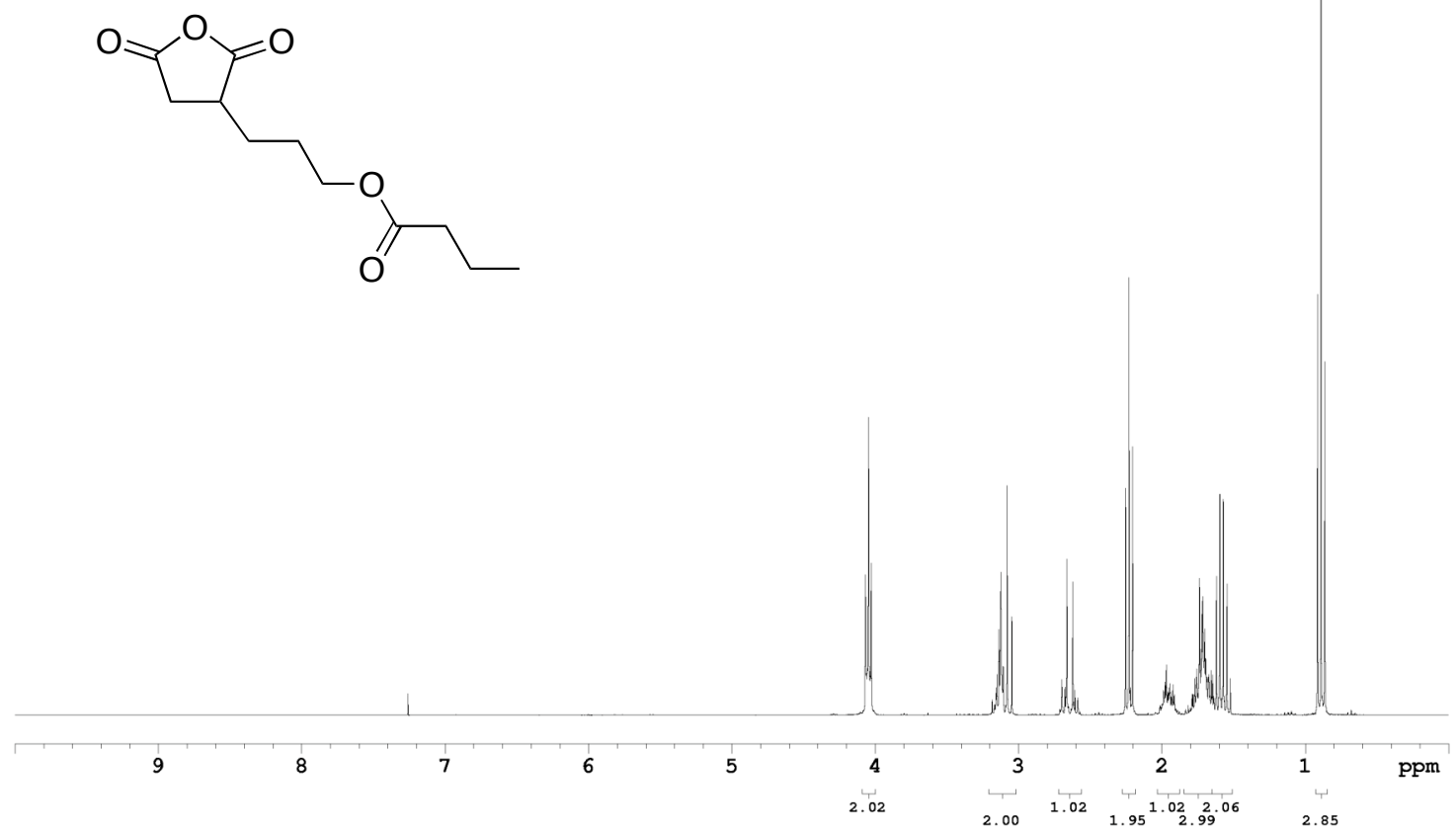

Figure S15. ${ }^{13} \mathrm{C}$ NMR spectrum of 25, 3-butyroxypropylsuccinic anhydride $\mathrm{CDCl}_{3}, 75 \mathrm{MHz}$ (Table 2, entry 11).

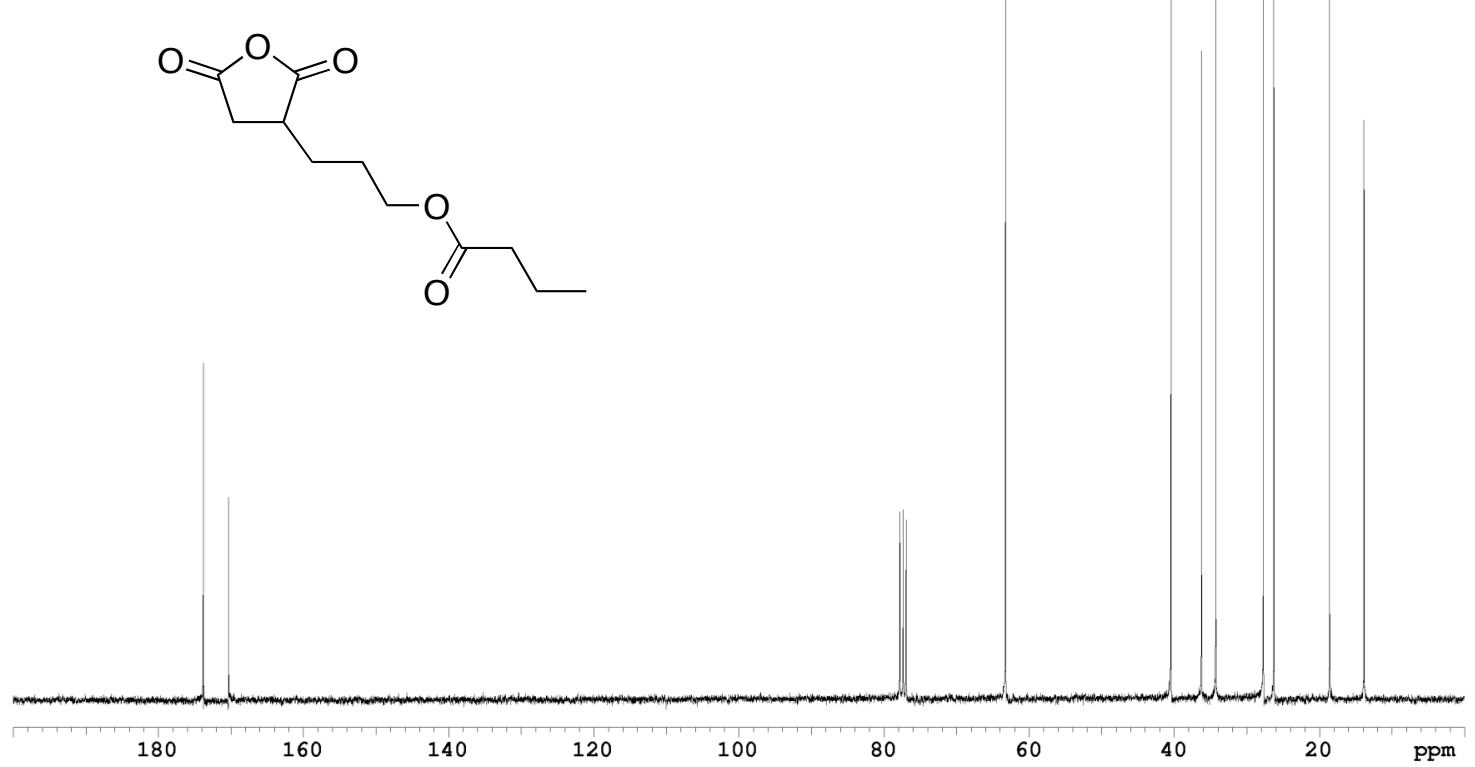


Figure S16. ${ }^{1} \mathrm{H}$ NMR spectrum of 27, 3-cyanopropylsuccinic anhydride $\mathrm{CDCl}_{3}, 300 \mathrm{MHz}$ (Table 2, entry 12).

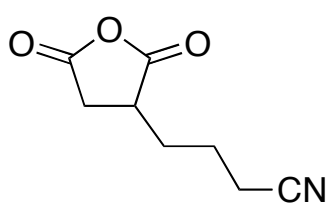
$\mathrm{CDCl}_{3}, 75 \mathrm{MHz}$ (Table 2, entry 12).
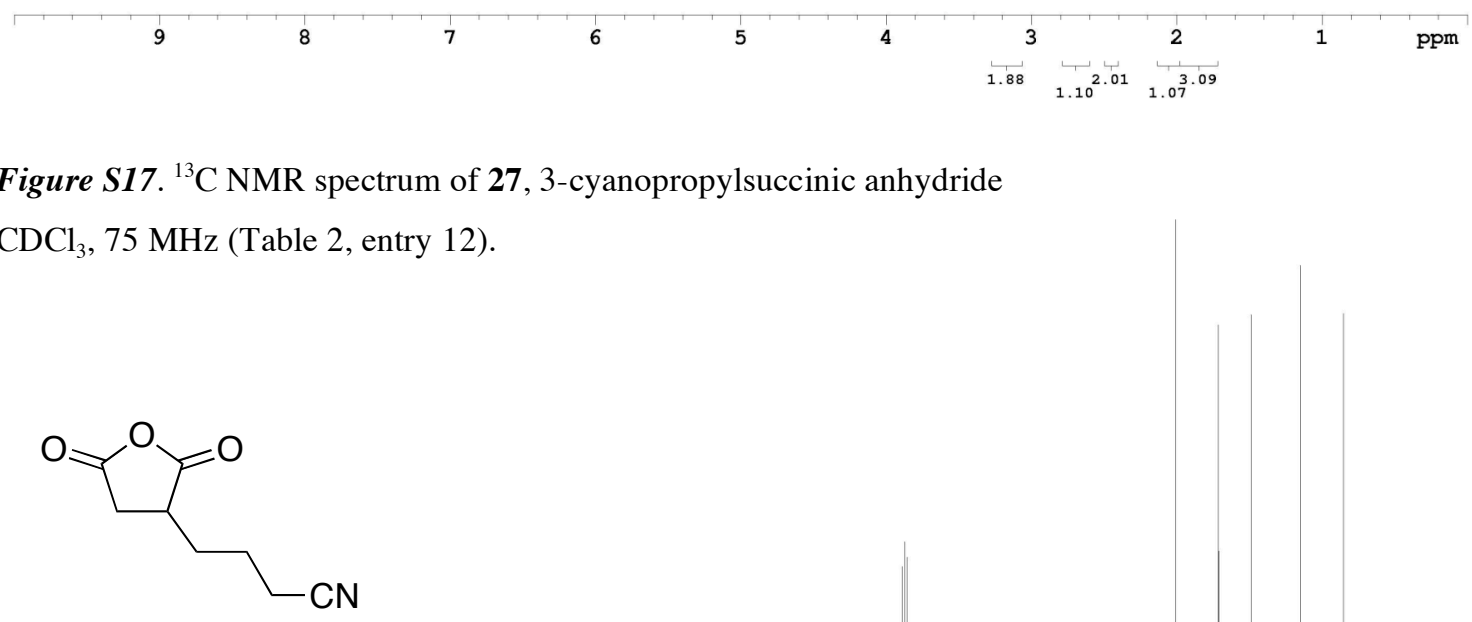

Figure S17. ${ }^{13} \mathrm{C}$ NMR spectrum of 27, 3-cyanopropylsuccinic anhydride

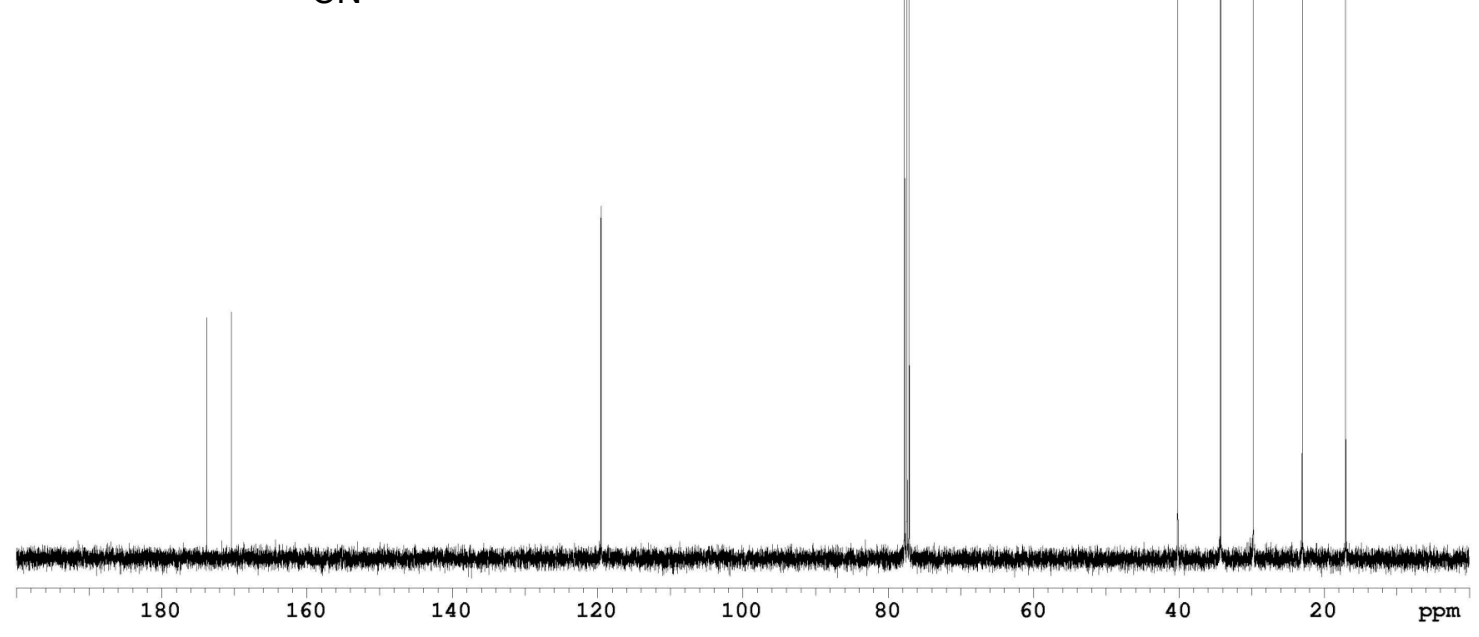


Figure S18. ${ }^{1} \mathrm{H}$ NMR spectrum of 29, 9-(succinic anhydridyl)- $N, N$-dimethylnonanamide $\mathrm{CDCl}_{3}, 300 \mathrm{MHz}$ (Table 2, entry 13).

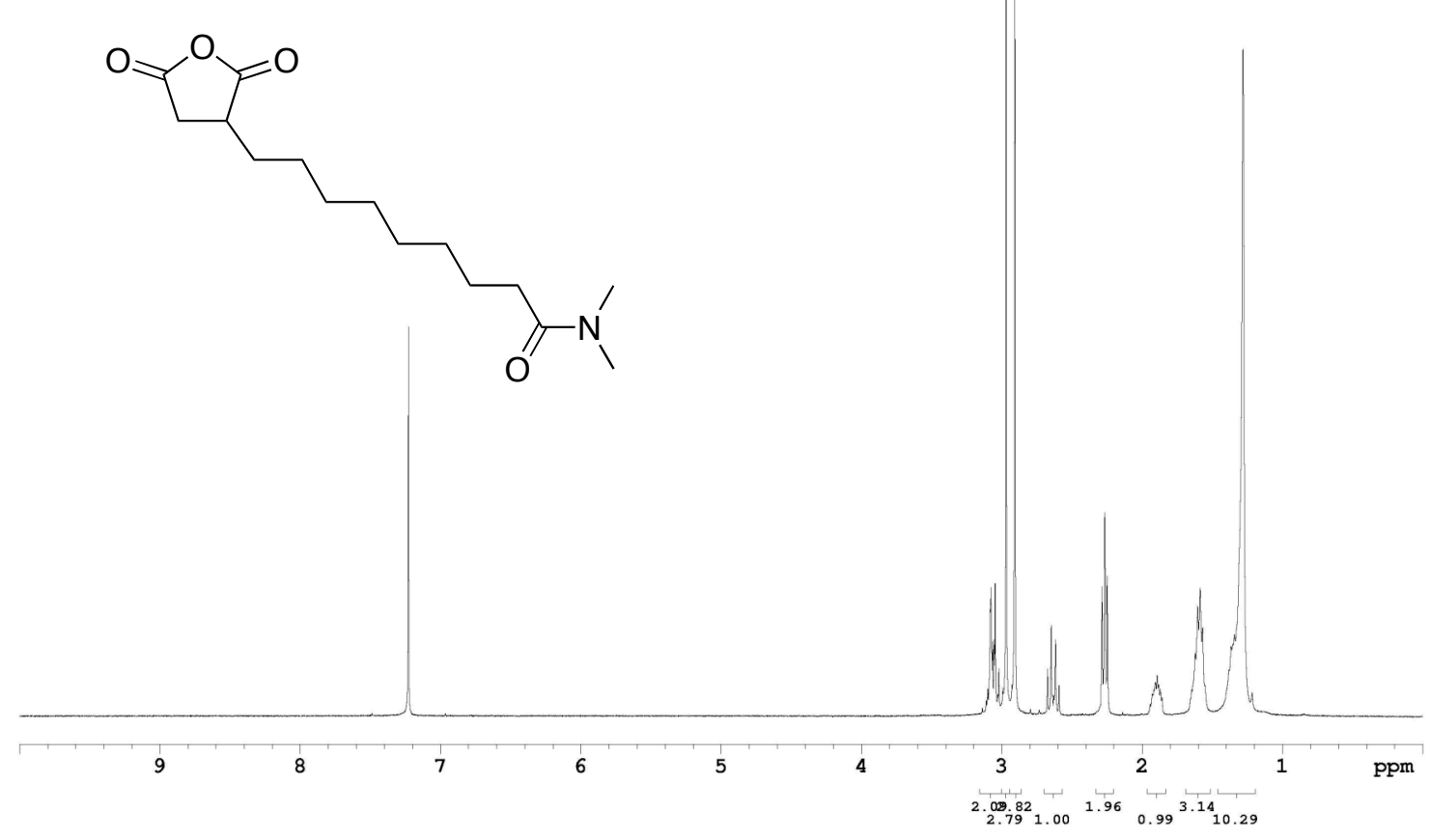

Figure S19. ${ }^{13} \mathrm{C}$ NMR spectrum of 29, 9-(succinic anhydridyl)- $N, N$-dimethylnonanamide $\mathrm{CDCl}_{3}, 75 \mathrm{MHz}$ (Table 2, entry 13).

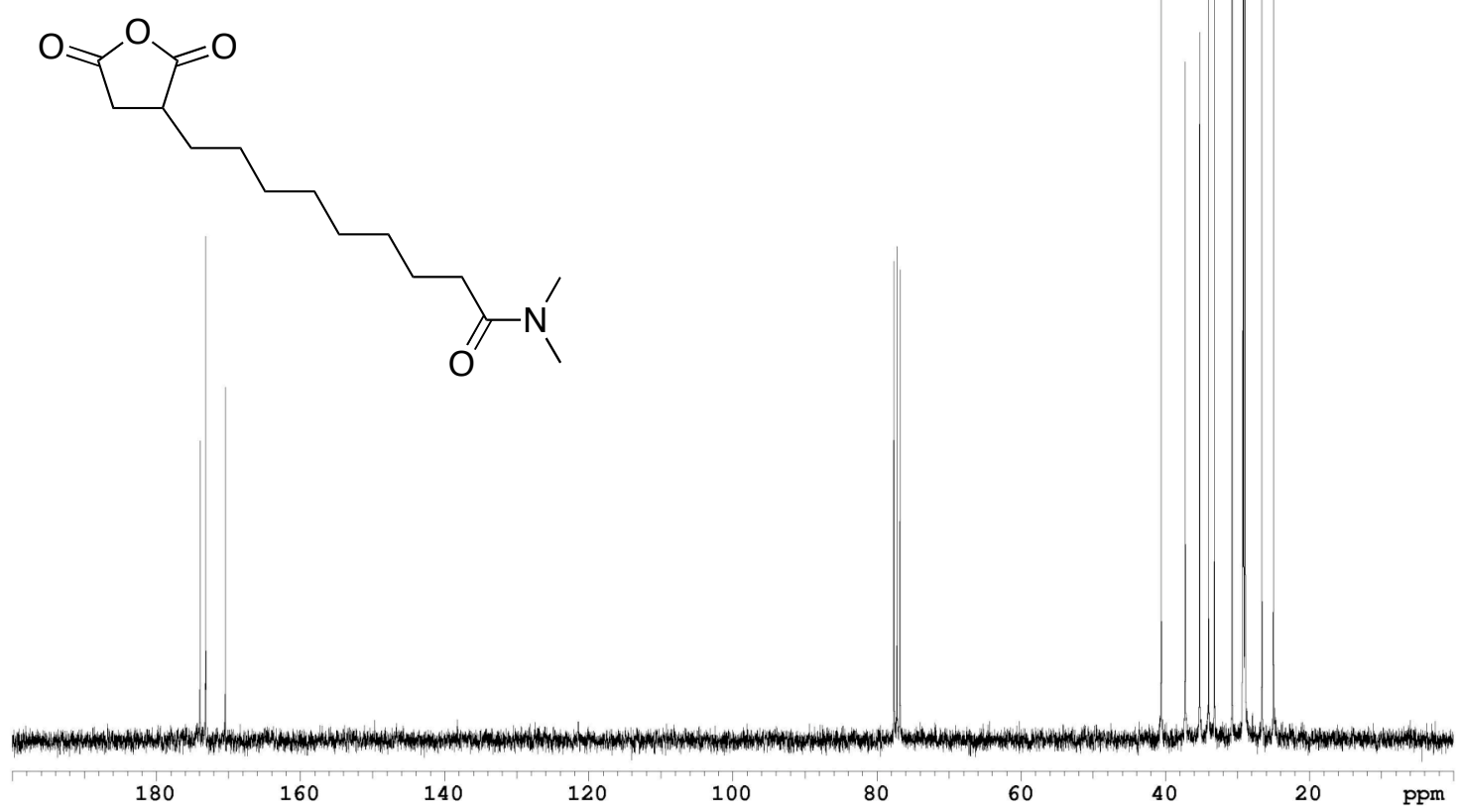


Figure S20. ${ }^{1} \mathrm{H}$ NMR spectrum of $\mathbf{3 3}$, hex-5-enylsuccinic anhydride $\mathrm{CDCl}_{3}, 300 \mathrm{MHz}$ (Table 2, entry 15).

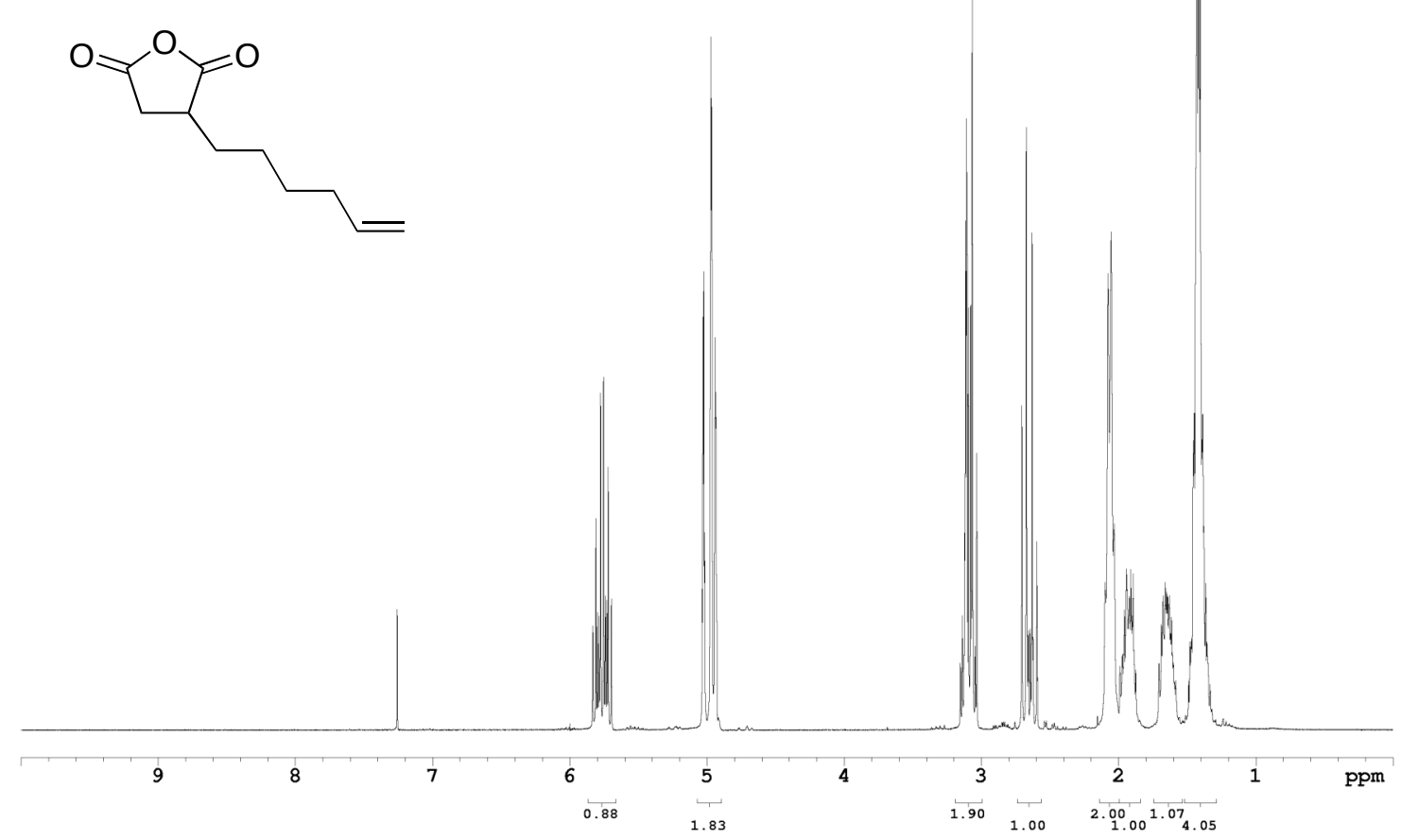

Figure S21. ${ }^{13} \mathrm{C}$ NMR spectrum of 33, hex-5-enylsuccinic anhydride $\mathrm{CDCl}_{3}, 75 \mathrm{MHz}$ (Table 2, entry 15).
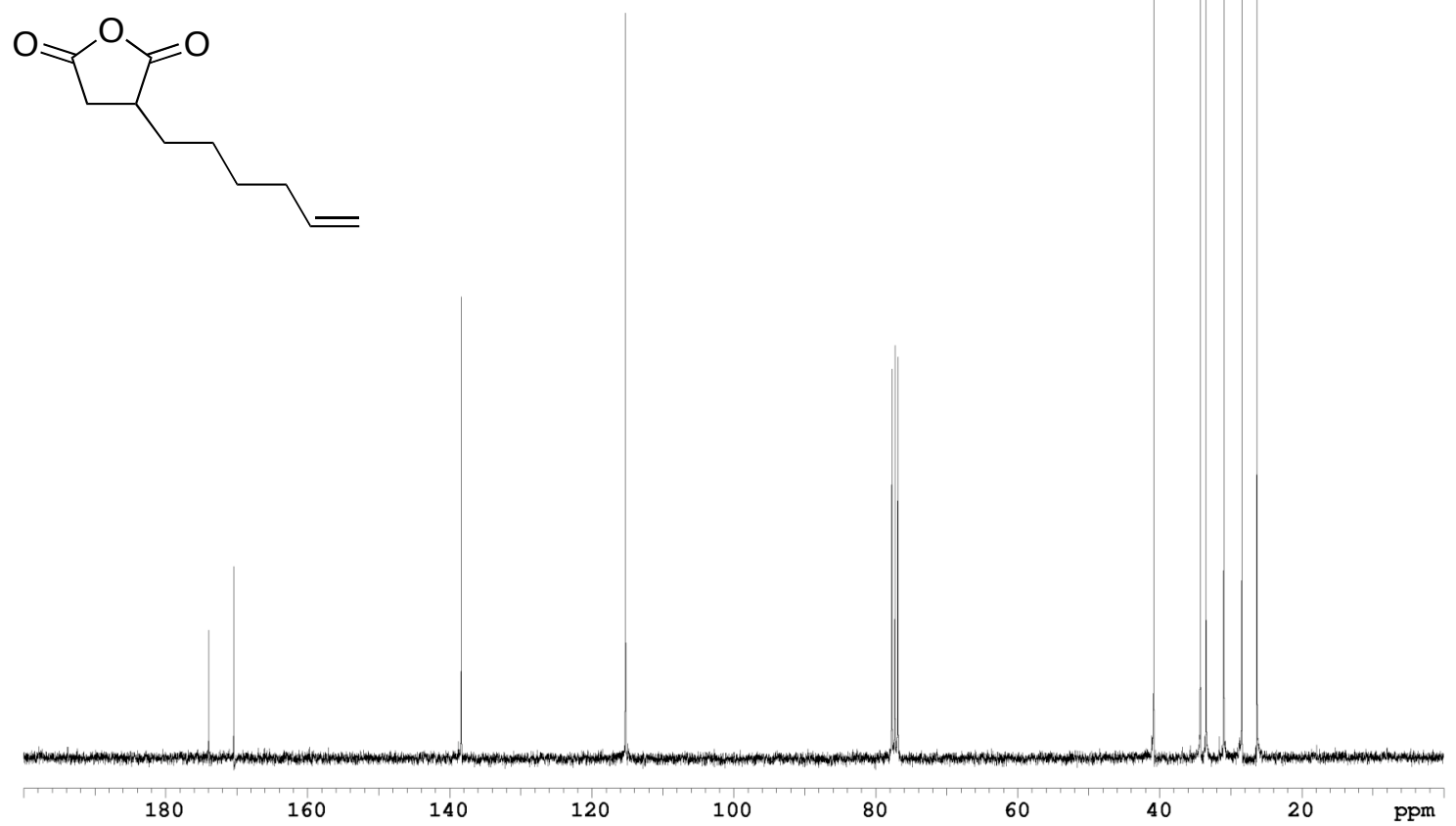
Figure S22. ${ }^{1} \mathrm{H}$ NMR spectrum of 39, rac/meso-1,4-bis(succinic anhydridyl)butane Acetone- $d_{6}, 300 \mathrm{MHz}$ (Table 2, entry 18).

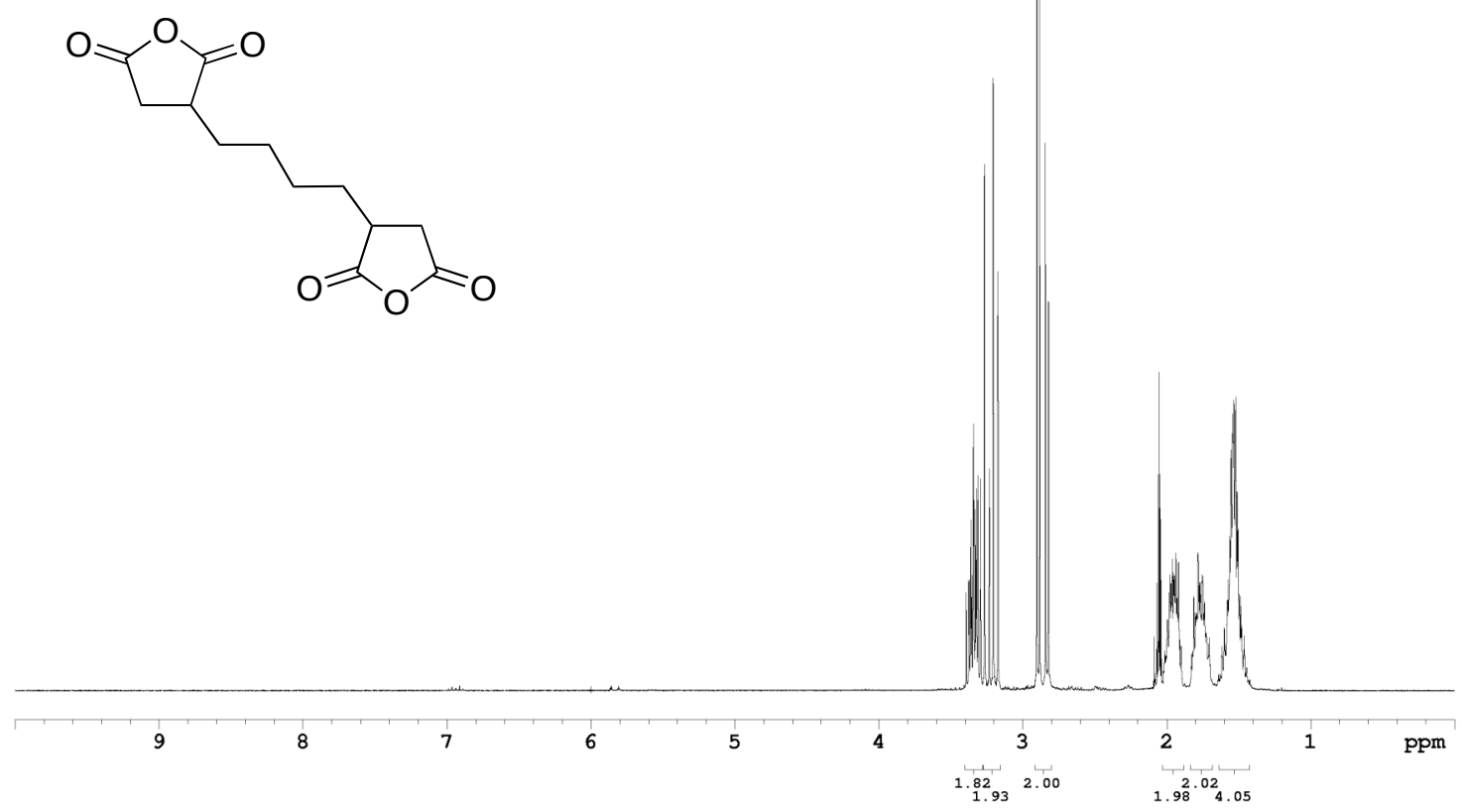

Figure S23. ${ }^{13} \mathrm{C}$ NMR spectrum of 39, rac/meso-1,4-bis(succinic anhydridyl)butane Acetone- $d_{6}, 75 \mathrm{MHz}$ (Table 2, entry 18).

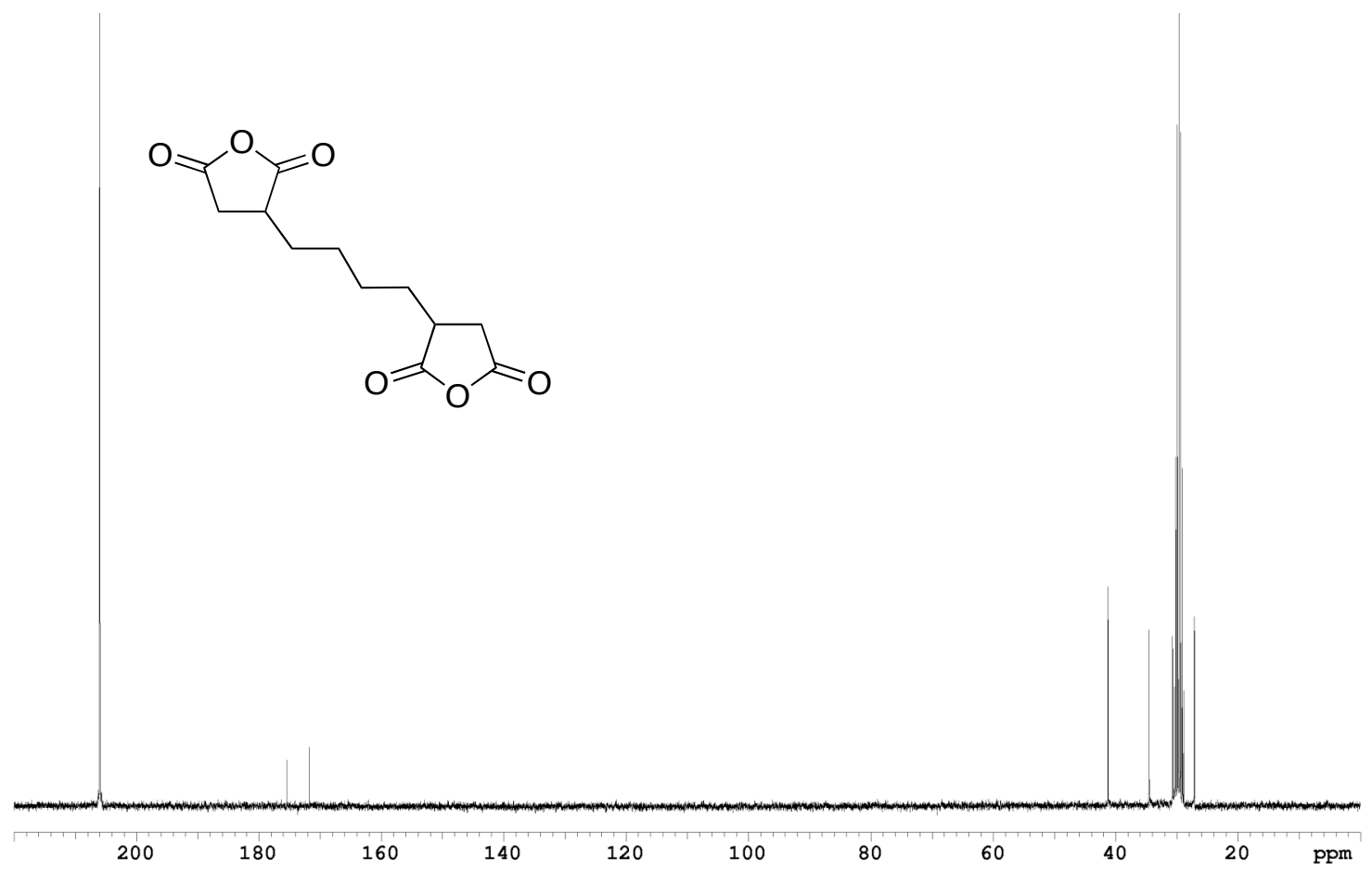


Figure S24. ${ }^{1} \mathrm{H}$ NMR spectrum of 47, trans-2-methyl-3-pentylsuccinic anhydride $\mathrm{CDCl}_{3}, 300 \mathrm{MHz}$ (Table 3, entry 4).

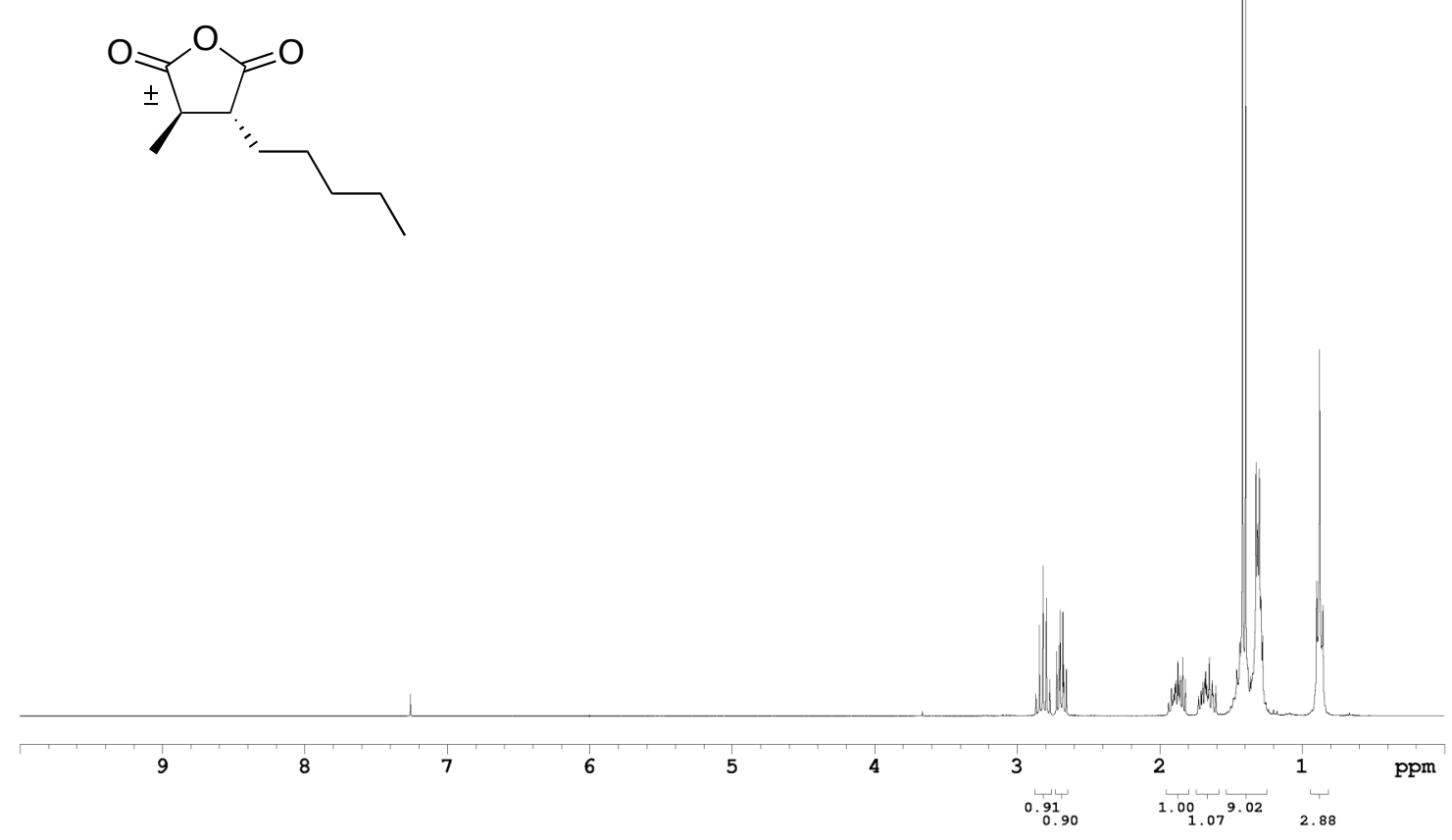

Figure S25. ${ }^{13} \mathrm{C}$ NMR spectrum of 47, trans-2-methyl-3-pentylsuccinic anhydride $\mathrm{CDCl}_{3}, 75 \mathrm{MHz}$ (Table 3, entry 4).

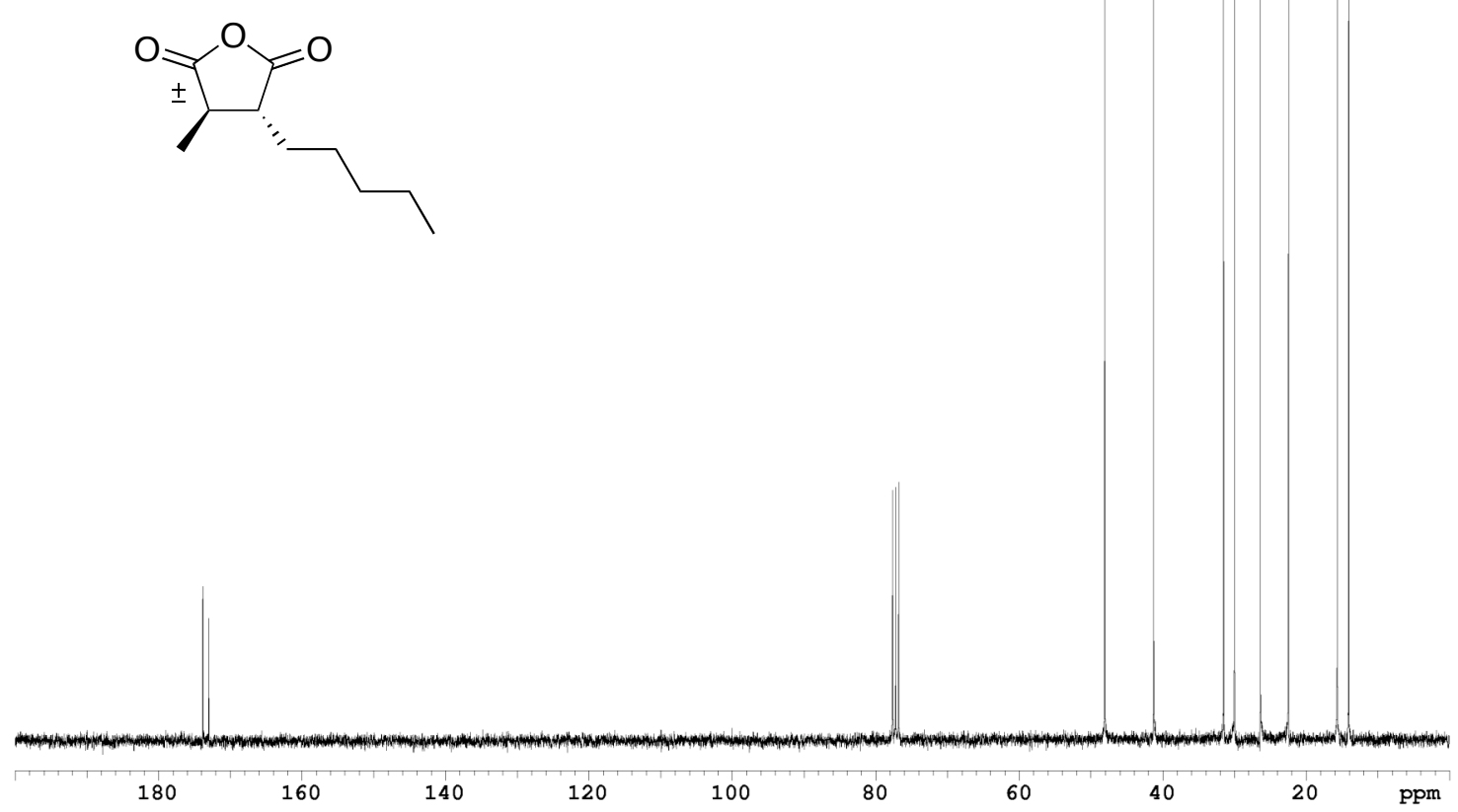




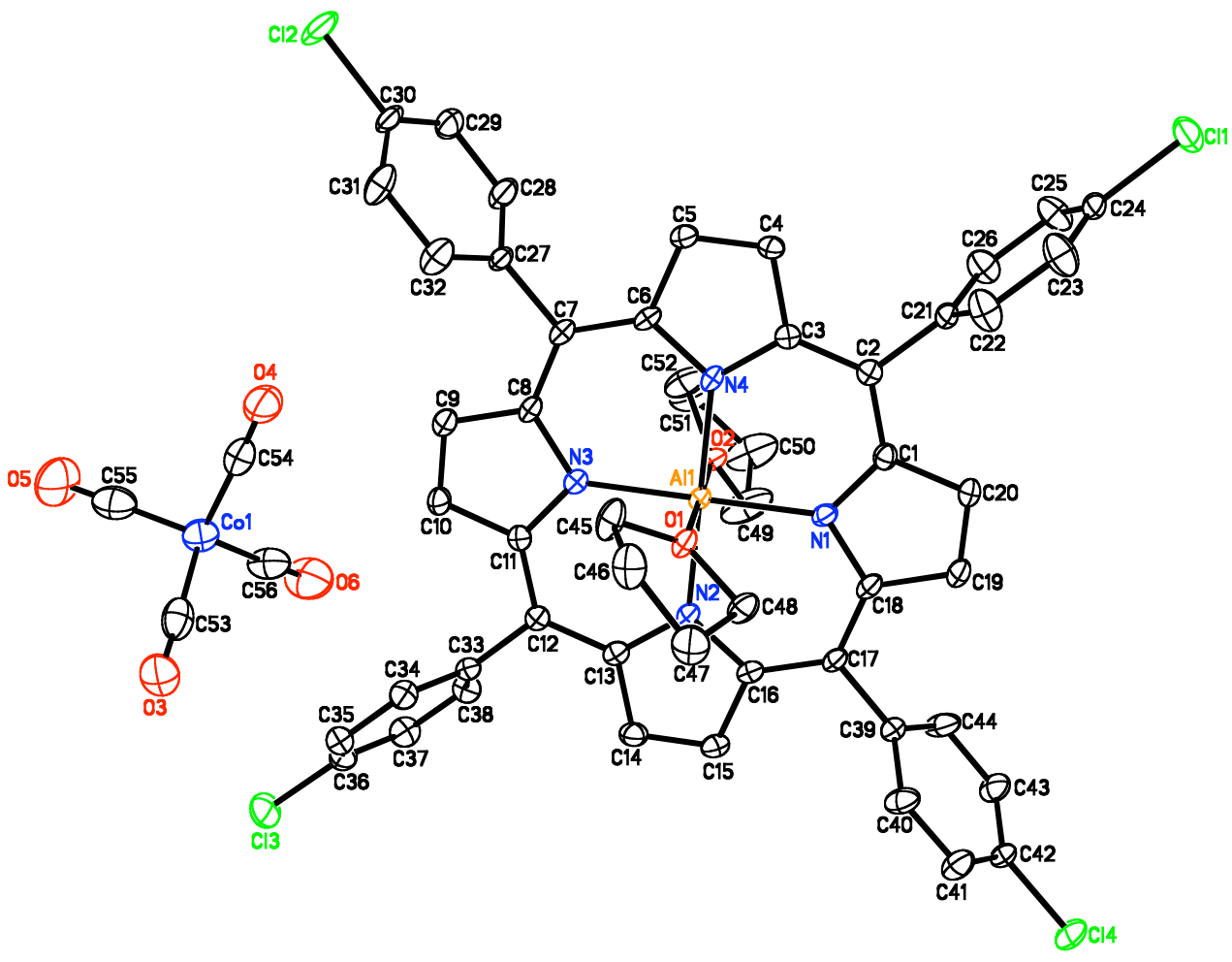

Figure S26. A view of the molecular structure of $\left[(\mathrm{ClTPP}) \mathrm{Al}(\mathrm{THF})_{2}\right]^{+}\left[\mathrm{Co}(\mathrm{CO})_{4}\right]^{-}$, (1) (hydrogen atoms omitted for clarity). Displacement ellipsoids are drawn at the $40 \%$ probability level.

Figure S27. Photograph of 1.

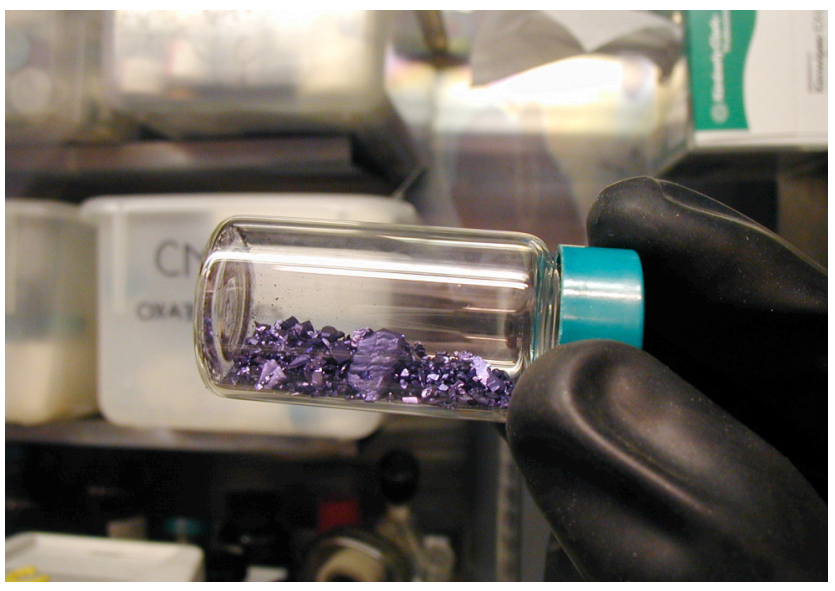


Table S1. Crystal data and structure refinement for 1.

Identification code

Empirical formula

Formula weight

Temperature

Wavelength

Crystal system

Space group

Unit cell dimensions

Volume

Z

Density (calculated)

Absorption coefficient

$\mathrm{F}(000)$

Crystal size

Theta range for data collection

Index ranges

Reflections collected

Independent reflections

Completeness to theta $=25.92^{\circ}$

Absorption correction

Max. and min. transmission

Refinement method

Data / restraints / parameters

Goodness-of-fit on $\mathrm{F}^{2}$

Final R indices [I $>2 \operatorname{sigma}(\mathrm{I})]$

$\mathrm{R}$ indices (all data)

Largest diff. peak and hole jr4

C56 H40 Al Cl4 Co N4 O6

1092.63

173(2) K

$0.71073 \AA$

Monoclinic

$\mathrm{P} 2(1) / \mathrm{n}$

$\mathrm{a}=11.9296(6) \AA$

$\alpha=90^{\circ}$

$\mathrm{b}=22.6420(12) \AA$

$\beta=102.446(2)^{\circ}$

$\mathrm{c}=20.4696(10) \AA$

$\gamma=90^{\circ}$

5399.1(5) $\AA^{3}$

4

$1.344 \mathrm{Mg} / \mathrm{m}^{3}$

$0.585 \mathrm{~mm}^{-1}$

2240

$0.45 \times 0.40 \times 0.30 \mathrm{~mm}^{3}$

11.39 to $25.92^{\circ}$.

$-14<=\mathrm{h}<=14,-27<=\mathrm{k}<=27,-25<=1<=20$

36958

$9511[\mathrm{R}(\mathrm{int})=0.0536]$

$90.3 \%$

Semiempirical by SADABS

0.8441 and 0.7788

Full-matrix least-squares on $\mathrm{F}^{2}$

$9511 / 220 / 783$

1.123

$\mathrm{R} 1=0.0593, \mathrm{wR} 2=0.1634$

$\mathrm{R} 1=0.0854, \mathrm{wR} 2=0.1727$

0.518 and $-0.567 \mathrm{e} \cdot \AA^{-3}$ 
Table S2. Atomic coordinates ( x 10 $0^{4}$ ) and equivalent isotropic displacement parameters $\left(\AA^{2} \times 10^{3}\right)$ for $\mathbf{1} . \mathrm{U}(\mathrm{eq})$ is defined as one third of the trace of the orthogonalized $\mathrm{U}^{\mathrm{ij}}$ tensor.

\begin{tabular}{|c|c|c|c|c|}
\hline & $\mathrm{x}$ & $\mathrm{y}$ & $\mathrm{z}$ & $\mathrm{U}(\mathrm{eq})$ \\
\hline $\mathrm{Co}(1)$ & $939(1)$ & $2452(1)$ & $532(1)$ & $43(1)$ \\
\hline $\operatorname{Al}(1)$ & $1(1)$ & $-845(1)$ & $2533(1)$ & $16(1)$ \\
\hline $\mathrm{Cl}(1)$ & $36(1)$ & $-5011(1)$ & $2516(1)$ & $44(1)$ \\
\hline $\mathrm{Cl}(2)$ & $3807(1)$ & $-879(1)$ & $-1065(1)$ & $49(1)$ \\
\hline $\mathrm{Cl}(3)$ & $-56(1)$ & $3321(1)$ & $2461(1)$ & $45(1)$ \\
\hline $\mathrm{Cl}(4)$ & $-3707(1)$ & $-939(1)$ & $6170(1)$ & $40(1)$ \\
\hline $\mathrm{O}(1)$ & $1489(2)$ & $-821(1)$ & $3193(1)$ & $22(1)$ \\
\hline $\mathrm{O}(2)$ & $-1493(2)$ & $-849(1)$ & $1878(1)$ & $21(1)$ \\
\hline $\mathrm{O}(3)$ & 2347(3) & $2907(1)$ & $1775(1)$ & $53(1)$ \\
\hline $\mathrm{O}(4)$ & $1820(4)$ & $1297(1)$ & $298(2)$ & $72(1)$ \\
\hline $\mathrm{O}(5)$ & $1200(4)$ & $3223(2)$ & $-555(2)$ & $81(1)$ \\
\hline $\mathrm{O}(6)$ & $-1490(3)$ & $2426(2)$ & $576(2)$ & $79(1)$ \\
\hline $\mathrm{N}(1)$ & $-570(2)$ & $-1471(1)$ & $3066(1)$ & $19(1)$ \\
\hline $\mathrm{N}(2)$ & $-614(2)$ & $-226(1)$ & $3061(1)$ & $18(1)$ \\
\hline $\mathrm{N}(3)$ & $568(2)$ & $-221(1)$ & 1995(1) & $19(1)$ \\
\hline $\mathrm{N}(4)$ & $591(2)$ & $-1466(1)$ & 1999(1) & $19(1)$ \\
\hline$C(1)$ & $-474(2)$ & $-2070(1)$ & 2999(1) & $19(1)$ \\
\hline$C(2)$ & $19(2)$ & $-2355(1)$ & 2531(1) & $19(1)$ \\
\hline$C(3)$ & $508(2)$ & $-2066(1)$ & $2067(1)$ & $19(1)$ \\
\hline$C(4)$ & $1013(2)$ & $-2364(2)$ & $1582(1)$ & $23(1)$ \\
\hline$C(5)$ & 1412(3) & $-1943(1)$ & $1222(2)$ & $23(1)$ \\
\hline$C(6)$ & $1152(2)$ & $-1379(1)$ & $1478(1)$ & $20(1)$ \\
\hline$C(7)$ & $1403(2)$ & $-843(1)$ & $1229(1)$ & $20(1)$ \\
\hline$C(8)$ & $1104(2)$ & $-300(1)$ & $1469(1)$ & $20(1)$ \\
\hline$C(9)$ & 1311(3) & $262(1)$ & $1193(2)$ & $26(1)$ \\
\hline$C(10)$ & $903(3)$ & $679(2)$ & $1548(2)$ & $25(1)$ \\
\hline $\mathrm{C}(11)$ & $455(2)$ & $380(1)$ & $2052(1)$ & 19(1) \\
\hline$C(12)$ & $-52(2)$ & $669(1)$ & $2518(1)$ & $19(1)$ \\
\hline$C(13)$ & $-544(2)$ & $378(1)$ & 2992(1) & $20(1)$ \\
\hline$C(14)$ & $-1035(3)$ & $671(1)$ & $3481(2)$ & $25(1)$ \\
\hline$C(15)$ & $-1425(3)$ & $249(1)$ & $3841(2)$ & $24(1)$ \\
\hline
\end{tabular}




\begin{tabular}{|c|c|c|c|c|}
\hline $\mathrm{C}(16)$ & $-1159(2)$ & $-311(1)$ & $3584(1)$ & $19(1)$ \\
\hline $\mathrm{C}(17)$ & $-1424(2)$ & $-855(1)$ & $3828(1)$ & $20(1)$ \\
\hline $\mathrm{C}(18)$ & $-1153(2)$ & $-1391(1)$ & $3577(1)$ & $21(1)$ \\
\hline$C(19)$ & $-1403(3)$ & $-1953(1)$ & $3832(2)$ & $26(1)$ \\
\hline $\mathrm{C}(20)$ & $-987(3)$ & $-2372(2)$ & $3474(2)$ & $25(1)$ \\
\hline $\mathrm{C}(21)$ & $25(2)$ & $-3016(1)$ & $2534(1)$ & $22(1)$ \\
\hline $\mathrm{C}(22)$ & $901(3)$ & $-3332(1)$ & 2941(2) & $37(1)$ \\
\hline $\mathrm{C}(23)$ & $901(3)$ & $-3944(2)$ & 2942(2) & $41(1)$ \\
\hline$C(24)$ & $24(3)$ & $-4244(1)$ & $2527(2)$ & $26(1)$ \\
\hline$C(25)$ & $-853(3)$ & $-3943(2)$ & $2124(2)$ & $38(1)$ \\
\hline$C(26)$ & $-853(3)$ & $-3333(1)$ & $2126(2)$ & $32(1)$ \\
\hline $\mathrm{C}(27)$ & $2004(2)$ & $-836(1)$ & $655(1)$ & $21(1)$ \\
\hline $\mathrm{C}(28)$ & $1453(7)$ & $-1008(5)$ & $28(4)$ & $33(2)$ \\
\hline $\mathrm{C}\left(28^{\prime}\right)$ & $1347(18)$ & $-743(12)$ & $-9(11)$ & $31(4)$ \\
\hline$C(29)$ & $2007(7)$ & $-1025(5)$ & $-503(4)$ & $37(2)$ \\
\hline $\mathrm{C}\left(29^{\prime}\right)$ & 1906(16) & $-751(12)$ & $-519(9)$ & $30(4)$ \\
\hline $\mathrm{C}(30)$ & $3106(3)$ & $-852(1)$ & $-401(2)$ & $29(1)$ \\
\hline $\mathrm{C}(31)$ & $3727(6)$ & $-661(5)$ & $229(4)$ & $38(2)$ \\
\hline $\mathrm{C}\left(31^{\prime}\right)$ & $3671(14)$ & $-963(12)$ & $212(8)$ & $29(4)$ \\
\hline $\mathrm{C}(32)$ & $3159(7)$ & $-648(5)$ & $756(4)$ & $34(2)$ \\
\hline $\mathrm{C}\left(32^{\prime}\right)$ & $3141(15)$ & $-937(13)$ & $742(9)$ & $31(4)$ \\
\hline $\mathrm{C}(33)$ & $-60(2)$ & $1332(1)$ & $2515(1)$ & $22(1)$ \\
\hline $\mathrm{C}(34)$ & $959(3)$ & $1644(1)$ & $2681(2)$ & $26(1)$ \\
\hline $\mathrm{C}(35)$ & $966(3)$ & $2255(1)$ & $2674(2)$ & $29(1)$ \\
\hline $\mathrm{C}(36)$ & $-70(3)$ & $2549(1)$ & $2485(2)$ & $31(1)$ \\
\hline $\mathrm{C}(37)$ & $-1085(3)$ & $2254(1)$ & $2318(2)$ & $30(1)$ \\
\hline $\mathrm{C}(38)$ & $-1087(3)$ & $1643(1)$ & $2334(2)$ & $27(1)$ \\
\hline $\mathrm{C}(39)$ & $-2005(2)$ & $-864(1)$ & $4405(1)$ & $21(1)$ \\
\hline $\mathrm{C}(40)$ & $-1380(8)$ & $-741(4)$ & $5055(5)$ & $31(2)$ \\
\hline $\mathrm{C}\left(40^{\prime}\right)$ & $-1380(20)$ & $-991(13)$ & $5021(12)$ & $29(5)$ \\
\hline $\mathrm{C}(41)$ & $-1889(5)$ & $-764(4)$ & $5597(3)$ & $32(2)$ \\
\hline$C\left(41^{\prime}\right)$ & $-2006(18)$ & $-1050(12)$ & 5564(9) & $31(4)$ \\
\hline $\mathrm{C}(42)$ & $-3049(3)$ & $-916(1)$ & $5489(2)$ & $26(1)$ \\
\hline $\mathrm{C}(43)$ & $-3665(5)$ & $-1045(4)$ & 4872(3) & $32(2)$ \\
\hline$C\left(43^{\prime}\right)$ & $-3748(16)$ & $-748(15)$ & $4828(10)$ & $36(5)$ \\
\hline $\mathrm{C}(44)$ & $-3153(7)$ & $-1031(4)$ & 4329(4) & $36(2)$ \\
\hline
\end{tabular}




\begin{tabular}{lcccc}
$\mathrm{C}\left(44^{\prime}\right)$ & $-3182(17)$ & $-740(16)$ & $4325(10)$ & $38(5)$ \\
$\mathrm{C}(45)$ & $2591(3)$ & $-649(2)$ & $3047(2)$ & $35(1)$ \\
$\mathrm{C}(46)$ & $3471(3)$ & $-747(2)$ & $3685(2)$ & $37(1)$ \\
$\mathrm{C}(47)$ & $2802(3)$ & $-713(2)$ & $4225(2)$ & $40(1)$ \\
$\mathrm{C}(48)$ & $1641(3)$ & $-961(2)$ & $3907(2)$ & $28(1)$ \\
$\mathrm{C}(49)$ & $-2601(3)$ & $-722(3)$ & $2034(2)$ & $61(1)$ \\
$\mathrm{C}(50)$ & $-3469(3)$ & $-851(3)$ & $1421(2)$ & $64(1)$ \\
$\mathrm{C}(51)$ & $-1653(3)$ & $-959(2)$ & $1158(2)$ & $44(1)$ \\
$\mathrm{C}(52)$ & $-2853(3)$ & $-765(2)$ & $868(2)$ & $48(1)$ \\
$\mathrm{C}(53)$ & $1777(3)$ & $2722(2)$ & $1290(2)$ & $46(1)$ \\
$\mathrm{C}(54)$ & $1448(4)$ & $1747(2)$ & $391(2)$ & $50(1)$ \\
$\mathrm{C}(55)$ & $1081(4)$ & $2915(2)$ & $-126(2)$ & $56(1)$ \\
$\mathrm{C}(56)$ & $-536(4)$ & $2433(2)$ & $577(2)$ & $53(1)$ \\
& & & & \\
\hline
\end{tabular}


Table S3. Bond lengths $[\AA]$ and angles $\left[{ }^{\circ}\right]$ for $\mathbf{1}$.

\begin{tabular}{|c|c|c|c|}
\hline & & $C(3)-C(4)$ & $1.436(4)$ \\
\hline $\mathrm{Co}(1)-\mathrm{C}(55)$ & $1.744(6)$ & $C(4)-C(5)$ & $1.352(5)$ \\
\hline $\mathrm{Co}(1)-\mathrm{C}(54)$ & $1.753(5)$ & $C(5)-C(6)$ & $1.439(4)$ \\
\hline $\mathrm{Co}(1)-\mathrm{C}(53)$ & $1.763(5)$ & $\mathrm{C}(6)-\mathrm{C}(7)$ & $1.375(4)$ \\
\hline $\mathrm{Co}(1)-\mathrm{C}(56)$ & $1.783(5)$ & $C(7)-C(8)$ & $1.396(4)$ \\
\hline $\mathrm{Al}(1)-\mathrm{O}(2)$ & $1.9863(19)$ & $C(7)-C(27)$ & $1.503(4)$ \\
\hline $\mathrm{Al}(1)-\mathrm{O}(1)$ & $1.987(2)$ & $\mathrm{C}(8)-\mathrm{C}(9)$ & $1.435(4)$ \\
\hline $\operatorname{Al}(1)-\mathrm{N}(3)$ & $1.997(2)$ & $\mathrm{C}(9)-\mathrm{C}(10)$ & $1.344(5)$ \\
\hline $\mathrm{Al}(1)-\mathrm{N}(1)$ & $1.995(2)$ & $\mathrm{C}(10)-\mathrm{C}(11)$ & $1.430(4)$ \\
\hline $\mathrm{Al}(1)-\mathrm{N}(4)$ & $1.998(2)$ & $\mathrm{C}(11)-\mathrm{C}(12)$ & $1.398(4)$ \\
\hline $\mathrm{Al}(1)-\mathrm{N}(2)$ & $2.004(2)$ & $\mathrm{C}(12)-\mathrm{C}(13)$ & $1.402(4)$ \\
\hline $\mathrm{Cl}(1)-\mathrm{C}(24)$ & $1.737(3)$ & $\mathrm{C}(12)-\mathrm{C}(33)$ & $1.500(4)$ \\
\hline $\mathrm{Cl}(2)-\mathrm{C}(30)$ & $1.743(3)$ & $\mathrm{C}(13)-\mathrm{C}(14)$ & $1.427(4)$ \\
\hline $\mathrm{Cl}(3)-\mathrm{C}(36)$ & $1.749(3)$ & $\mathrm{C}(14)-\mathrm{C}(15)$ & $1.350(5)$ \\
\hline $\mathrm{Cl}(4)-\mathrm{C}(42)$ & $1.741(3)$ & $\mathrm{C}(15)-\mathrm{C}(16)$ & $1.434(4)$ \\
\hline $\mathrm{O}(1)-\mathrm{C}(45)$ & $1.462(4)$ & $\mathrm{C}(16)-\mathrm{C}(17)$ & $1.390(4)$ \\
\hline $\mathrm{O}(1)-\mathrm{C}(48)$ & $1.468(4)$ & $\mathrm{C}(17)-\mathrm{C}(18)$ & $1.384(4)$ \\
\hline $\mathrm{O}(2)-\mathrm{C}(49)$ & $1.454(4)$ & $\mathrm{C}(17)-\mathrm{C}(39)$ & $1.492(4)$ \\
\hline $\mathrm{O}(2)-\mathrm{C}(51)$ & $1.465(4)$ & $\mathrm{C}(18)-\mathrm{C}(19)$ & $1.431(4)$ \\
\hline $\mathrm{O}(3)-\mathrm{C}(53)$ & $1.154(5)$ & $C(19)-C(20)$ & $1.358(5)$ \\
\hline $\mathrm{O}(4)-\mathrm{C}(54)$ & $1.144(5)$ & $\mathrm{C}(21)-\mathrm{C}(26)$ & $1.392(4)$ \\
\hline $\mathrm{O}(5)-\mathrm{C}(55)$ & $1.154(6)$ & $\mathrm{C}(21)-\mathrm{C}(22)$ & $1.387(4)$ \\
\hline $\mathrm{O}(6)-\mathrm{C}(56)$ & $1.138(6)$ & $\mathrm{C}(22)-\mathrm{C}(23)$ & $1.386(5)$ \\
\hline $\mathrm{N}(1)-\mathrm{C}(1)$ & $1.370(4)$ & $\mathrm{C}(23)-\mathrm{C}(24)$ & $1.376(5)$ \\
\hline $\mathrm{N}(1)-\mathrm{C}(18)$ & $1.388(4)$ & $\mathrm{C}(24)-\mathrm{C}(25)$ & $1.367(5)$ \\
\hline $\mathrm{N}(2)-\mathrm{C}(13)$ & $1.380(4)$ & $\mathrm{C}(25)-\mathrm{C}(26)$ & $1.380(5)$ \\
\hline $\mathrm{N}(2)-\mathrm{C}(16)$ & $1.380(4)$ & $\mathrm{C}(27)-\mathrm{C}\left(32^{\prime}\right)$ & $1.350(18)$ \\
\hline $\mathrm{N}(3)-\mathrm{C}(11)$ & $1.375(4)$ & $\mathrm{C}(27)-\mathrm{C}(28)$ & $1.367(8)$ \\
\hline $\mathrm{N}(3)-\mathrm{C}(8)$ & $1.378(4)$ & $\mathrm{C}(27)-\mathrm{C}(32)$ & $1.415(9)$ \\
\hline $\mathrm{N}(4)-\mathrm{C}(3)$ & $1.372(4)$ & $\mathrm{C}(27)-\mathrm{C}\left(28^{\prime}\right)$ & $1.43(2)$ \\
\hline $\mathrm{N}(4)-\mathrm{C}(6)$ & $1.390(4)$ & $\mathrm{C}(28)-\mathrm{C}(29)$ & $1.388(11)$ \\
\hline$C(1)-C(2)$ & $1.386(4)$ & $\mathrm{C}\left(28^{\prime}\right)-\mathrm{C}\left(29^{\prime}\right)$ & $1.36(3)$ \\
\hline$C(1)-C(20)$ & $1.429(4)$ & $\mathrm{C}(29)-\mathrm{C}(30)$ & $1.341(8)$ \\
\hline$C(2)-C(3)$ & $1.383(4)$ & $\mathrm{C}\left(29^{\prime}\right)-\mathrm{C}(30)$ & $1.42(2)$ \\
\hline$C(2)-C(21)$ & $1.496(4)$ & $\mathrm{C}(30)-\mathrm{C}\left(31^{\prime}\right)$ & $1.315(16)$ \\
\hline
\end{tabular}




\begin{tabular}{|c|c|c|c|}
\hline$C(30)-C(31)$ & $1.410(9)$ & $\mathrm{O}(1)-\mathrm{Al}(1)-\mathrm{N}(3)$ & $89.79(9)$ \\
\hline$C(31)-C(32)$ & $1.392(10)$ & $\mathrm{O}(2)-\mathrm{Al}(1)-\mathrm{N}(1)$ & $90.18(9)$ \\
\hline $\mathrm{C}\left(31^{\prime}\right)-\mathrm{C}\left(32^{\prime}\right)$ & $1.37(2)$ & $\mathrm{O}(1)-\mathrm{Al}(1)-\mathrm{N}(1)$ & $90.47(9)$ \\
\hline $\mathrm{C}(33)-\mathrm{C}(34)$ & $1.384(4)$ & $\mathrm{N}(3)-\mathrm{Al}(1)-\mathrm{N}(1)$ & $179.68(11)$ \\
\hline $\mathrm{C}(33)-\mathrm{C}(38)$ & $1.393(4)$ & $\mathrm{O}(2)-\mathrm{Al}(1)-\mathrm{N}(4)$ & $89.89(9)$ \\
\hline$C(34)-C(35)$ & $1.384(5)$ & $\mathrm{O}(1)-\mathrm{Al}(1)-\mathrm{N}(4)$ & $91.35(9)$ \\
\hline$C(35)-C(36)$ & $1.384(5)$ & $\mathrm{N}(3)-\mathrm{Al}(1)-\mathrm{N}(4)$ & $89.71(10)$ \\
\hline$C(36)-C(37)$ & $1.361(5)$ & $\mathrm{N}(1)-\mathrm{Al}(1)-\mathrm{N}(4)$ & $90.10(10)$ \\
\hline$C(37)-C(38)$ & $1.384(5)$ & $\mathrm{O}(2)-\mathrm{Al}(1)-\mathrm{N}(2)$ & $89.32(9)$ \\
\hline$C(39)-C\left(40^{\prime}\right)$ & $1.35(2)$ & $\mathrm{O}(1)-\mathrm{Al}(1)-\mathrm{N}(2)$ & $89.45(9)$ \\
\hline$C(39)-C(44)$ & $1.396(8)$ & $\mathrm{N}(3)-\mathrm{Al}(1)-\mathrm{N}(2)$ & $90.52(10)$ \\
\hline$C(39)-C(40)$ & $1.406(10)$ & $\mathrm{N}(1)-\mathrm{Al}(1)-\mathrm{N}(2)$ & $89.67(10)$ \\
\hline$C(39)-C\left(44^{\prime}\right)$ & $1.41(2)$ & $\mathrm{N}(4)-\mathrm{Al}(1)-\mathrm{N}(2)$ & $179.17(10)$ \\
\hline$C(40)-C(41)$ & $1.375(11)$ & $\mathrm{C}(45)-\mathrm{O}(1)-\mathrm{C}(48)$ & $109.8(2)$ \\
\hline $\mathrm{C}\left(40^{\prime}\right)-\mathrm{C}\left(41^{\prime}\right)$ & $1.47(3)$ & $\mathrm{C}(45)-\mathrm{O}(1)-\mathrm{Al}(1)$ & $125.35(17)$ \\
\hline$C(41)-C(42)$ & $1.397(8)$ & $\mathrm{C}(48)-\mathrm{O}(1)-\mathrm{Al}(1)$ & $124.88(17)$ \\
\hline$C\left(41^{\prime}\right)-C(42)$ & $1.258(19)$ & $\mathrm{C}(49)-\mathrm{O}(2)-\mathrm{C}(51)$ & $109.1(2)$ \\
\hline$C(42)-C(43)$ & $1.348(7)$ & $\mathrm{C}(49)-\mathrm{O}(2)-\mathrm{Al}(1)$ & $125.21(19)$ \\
\hline$C(42)-C\left(43^{\prime}\right)$ & $1.48(2)$ & $\mathrm{C}(51)-\mathrm{O}(2)-\mathrm{Al}(1)$ & $125.67(19)$ \\
\hline$C(43)-C(44)$ & $1.381(10)$ & $\mathrm{C}(1)-\mathrm{N}(1)-\mathrm{C}(18)$ & $105.7(2)$ \\
\hline $\mathrm{C}\left(43^{\prime}\right)-\mathrm{C}\left(44^{\prime}\right)$ & $1.35(3)$ & $\mathrm{C}(1)-\mathrm{N}(1)-\mathrm{Al}(1)$ & $127.06(18)$ \\
\hline$C(45)-C(46)$ & $1.506(5)$ & $\mathrm{C}(18)-\mathrm{N}(1)-\mathrm{Al}(1)$ & $127.26(19)$ \\
\hline$C(46)-C(47)$ & $1.498(6)$ & $\mathrm{C}(13)-\mathrm{N}(2)-\mathrm{C}(16)$ & $105.5(2)$ \\
\hline$C(47)-C(48)$ & $1.507(5)$ & $\mathrm{C}(13)-\mathrm{N}(2)-\mathrm{Al}(1)$ & $126.97(18)$ \\
\hline$C(49)-C(50)$ & $1.474(6)$ & $\mathrm{C}(16)-\mathrm{N}(2)-\mathrm{Al}(1)$ & $127.51(18)$ \\
\hline$C(50)-C(52)$ & $1.490(7)$ & $\mathrm{C}(11)-\mathrm{N}(3)-\mathrm{C}(8)$ & $105.4(2)$ \\
\hline \multirow[t]{2}{*}{$\mathrm{C}(51)-\mathrm{C}(52)$} & $1.492(6)$ & $\mathrm{C}(11)-\mathrm{N}(3)-\mathrm{Al}(1)$ & $127.06(18)$ \\
\hline & & $\mathrm{C}(8)-\mathrm{N}(3)-\mathrm{Al}(1)$ & $127.45(18)$ \\
\hline $\mathrm{C}(55)-\mathrm{Co}(1)-\mathrm{C}(54)$ & $108.99(18)$ & $\mathrm{C}(3)-\mathrm{N}(4)-\mathrm{C}(6)$ & $105.9(2)$ \\
\hline $\mathrm{C}(55)-\mathrm{Co}(1)-\mathrm{C}(53)$ & $109.87(19)$ & $\mathrm{C}(3)-\mathrm{N}(4)-\mathrm{Al}(1)$ & $126.86(18)$ \\
\hline $\mathrm{C}(54)-\mathrm{Co}(1)-\mathrm{C}(53)$ & $108.07(19)$ & $\mathrm{C}(6)-\mathrm{N}(4)-\mathrm{Al}(1)$ & $127.26(18)$ \\
\hline $\mathrm{C}(55)-\mathrm{Co}(1)-\mathrm{C}(56)$ & $108.4(2)$ & $\mathrm{N}(1)-\mathrm{C}(1)-\mathrm{C}(2)$ & $125.9(3)$ \\
\hline $\mathrm{C}(54)-\mathrm{Co}(1)-\mathrm{C}(56)$ & $111.5(2)$ & $\mathrm{N}(1)-\mathrm{C}(1)-\mathrm{C}(20)$ & $110.4(3)$ \\
\hline $\mathrm{C}(53)-\mathrm{Co}(1)-\mathrm{C}(56)$ & $110.01(18)$ & $\mathrm{C}(2)-\mathrm{C}(1)-\mathrm{C}(20)$ & $123.6(3)$ \\
\hline $\mathrm{O}(2)-\mathrm{Al}(1)-\mathrm{O}(1)$ & $178.60(9)$ & $\mathrm{C}(3)-\mathrm{C}(2)-\mathrm{C}(1)$ & $124.0(3)$ \\
\hline $\mathrm{O}(2)-\mathrm{Al}(1)-\mathrm{N}(3)$ & $89.56(9)$ & $C(3)-C(2)-C(21)$ & $118.2(2)$ \\
\hline
\end{tabular}




\begin{tabular}{|c|c|}
\hline$C(1)-C(2)-C(21)$ & $117.7(2)$ \\
\hline $\mathrm{N}(4)-\mathrm{C}(3)-\mathrm{C}(2)$ & $126.0(3)$ \\
\hline $\mathrm{N}(4)-\mathrm{C}(3)-\mathrm{C}(4)$ & $110.3(2)$ \\
\hline$C(2)-C(3)-C(4)$ & $123.7(3)$ \\
\hline$C(5)-C(4)-C(3)$ & 107.1(3) \\
\hline$C(4)-C(5)-C(6)$ & $107.4(3)$ \\
\hline $\mathrm{C}(7)-\mathrm{C}(6)-\mathrm{N}(4)$ & $126.0(2)$ \\
\hline$C(7)-C(6)-C(5)$ & $124.6(2)$ \\
\hline $\mathrm{N}(4)-\mathrm{C}(6)-\mathrm{C}(5)$ & $109.4(2)$ \\
\hline$C(6)-C(7)-C(8)$ & $123.7(2)$ \\
\hline$C(6)-C(7)-C(27)$ & $118.4(2)$ \\
\hline$C(8)-C(7)-C(27)$ & $117.9(2)$ \\
\hline $\mathrm{N}(3)-\mathrm{C}(8)-\mathrm{C}(7)$ & $125.9(2)$ \\
\hline $\mathrm{N}(3)-\mathrm{C}(8)-\mathrm{C}(9)$ & $109.9(2)$ \\
\hline $\mathrm{C}(7)-\mathrm{C}(8)-\mathrm{C}(9)$ & $124.3(2)$ \\
\hline$C(10)-C(9)-C(8)$ & $107.3(3)$ \\
\hline $\mathrm{C}(9)-\mathrm{C}(10)-\mathrm{C}(11)$ & $107.0(3)$ \\
\hline $\mathrm{N}(3)-\mathrm{C}(11)-\mathrm{C}(12)$ & $125.9(3)$ \\
\hline $\mathrm{N}(3)-\mathrm{C}(11)-\mathrm{C}(10)$ & $110.4(2)$ \\
\hline $\mathrm{C}(12)-\mathrm{C}(11)-\mathrm{C}(10)$ & $123.7(3)$ \\
\hline $\mathrm{C}(11)-\mathrm{C}(12)-\mathrm{C}(13)$ & $124.0(3)$ \\
\hline $\mathrm{C}(11)-\mathrm{C}(12)-\mathrm{C}(33)$ & $118.0(2)$ \\
\hline $\mathrm{C}(13)-\mathrm{C}(12)-\mathrm{C}(33)$ & $118.0(2)$ \\
\hline $\mathrm{N}(2)-\mathrm{C}(13)-\mathrm{C}(12)$ & $125.5(3)$ \\
\hline $\mathrm{N}(2)-\mathrm{C}(13)-\mathrm{C}(14)$ & $110.2(2)$ \\
\hline $\mathrm{C}(12)-\mathrm{C}(13)-\mathrm{C}(14)$ & $124.3(3)$ \\
\hline $\mathrm{C}(15)-\mathrm{C}(14)-\mathrm{C}(13)$ & 107.2(3) \\
\hline $\mathrm{C}(14)-\mathrm{C}(15)-\mathrm{C}(16)$ & 107.2(3) \\
\hline $\mathrm{N}(2)-\mathrm{C}(16)-\mathrm{C}(17)$ & $125.7(2)$ \\
\hline$N(2)-C(16)-C(15)$ & $109.9(2)$ \\
\hline $\mathrm{C}(17)-\mathrm{C}(16)-\mathrm{C}(15)$ & $124.4(3)$ \\
\hline $\mathrm{C}(18)-\mathrm{C}(17)-\mathrm{C}(16)$ & $123.7(2)$ \\
\hline $\mathrm{C}(18)-\mathrm{C}(17)-\mathrm{C}(39)$ & $117.8(2)$ \\
\hline $\mathrm{C}(16)-\mathrm{C}(17)-\mathrm{C}(39)$ & $118.5(2)$ \\
\hline $\mathrm{N}(1)-\mathrm{C}(18)-\mathrm{C}(17)$ & $126.1(2)$ \\
\hline $\mathrm{N}(1)-\mathrm{C}(18)-\mathrm{C}(19)$ & $109.7(2)$ \\
\hline
\end{tabular}

\begin{tabular}{|c|c|}
\hline $\mathrm{C}(17)-\mathrm{C}(18)-\mathrm{C}(19)$ & 124.1(3) \\
\hline $\mathrm{C}(20)-\mathrm{C}(19)-\mathrm{C}(18)$ & 107.1(3) \\
\hline$C(19)-C(20)-C(1)$ & $107.0(3)$ \\
\hline$C(26)-C(21)-C(22)$ & $117.7(3)$ \\
\hline$C(26)-C(21)-C(2)$ & $120.8(3)$ \\
\hline$C(22)-C(21)-C(2)$ & $121.4(3)$ \\
\hline$C(23)-C(22)-C(21)$ & 121.1(3) \\
\hline $\mathrm{C}(24)-\mathrm{C}(23)-\mathrm{C}(22)$ & $119.6(3)$ \\
\hline$C(25)-C(24)-C(23)$ & $120.5(3)$ \\
\hline $\mathrm{C}(25)-\mathrm{C}(24)-\mathrm{Cl}(1)$ & $119.9(2)$ \\
\hline $\mathrm{C}(23)-\mathrm{C}(24)-\mathrm{Cl}(1)$ & $119.6(2)$ \\
\hline$C(24)-C(25)-C(26)$ & $119.9(3)$ \\
\hline$C(25)-C(26)-C(21)$ & $121.2(3)$ \\
\hline$C\left(32^{\prime}\right)-C(27)-C(28)$ & $110.0(9)$ \\
\hline $\mathrm{C}\left(32^{\prime}\right)-\mathrm{C}(27)-\mathrm{C}(32)$ & $27.3(9)$ \\
\hline $\mathrm{C}(28)-\mathrm{C}(27)-\mathrm{C}(32)$ & $118.6(5)$ \\
\hline $\mathrm{C}\left(32^{\prime}\right)-\mathrm{C}(27)-\mathrm{C}\left(28^{\prime}\right)$ & $118.6(11)$ \\
\hline $\mathrm{C}(28)-\mathrm{C}(27)-\mathrm{C}\left(28^{\prime}\right)$ & $25.3(7)$ \\
\hline $\mathrm{C}(32)-\mathrm{C}(27)-\mathrm{C}\left(28^{\prime}\right)$ & $114.2(9)$ \\
\hline$C\left(32^{\prime}\right)-C(27)-C(7)$ & $122.1(8)$ \\
\hline $\mathrm{C}(28)-\mathrm{C}(27)-\mathrm{C}(7)$ & $121.2(4)$ \\
\hline$C(32)-C(27)-C(7)$ & $120.2(4)$ \\
\hline$C\left(28^{\prime}\right)-C(27)-C(7)$ & 119.3(9) \\
\hline $\mathrm{C}(27)-\mathrm{C}(28)-\mathrm{C}(29)$ & $121.9(6)$ \\
\hline $\mathrm{C}\left(29^{\prime}\right)-\mathrm{C}\left(28^{\prime}\right)-\mathrm{C}(27)$ & 118.1(16) \\
\hline $\mathrm{C}(30)-\mathrm{C}(29)-\mathrm{C}(28)$ & $119.0(7)$ \\
\hline $\mathrm{C}\left(28^{\prime}\right)-\mathrm{C}\left(29^{\prime}\right)-\mathrm{C}(30)$ & $121.1(15)$ \\
\hline $\mathrm{C}\left(31^{\prime}\right)-\mathrm{C}(30)-\mathrm{C}(29)$ & 112.1(8) \\
\hline $\mathrm{C}\left(31^{\prime}\right)-\mathrm{C}(30)-\mathrm{C}(31)$ & $28.9(8)$ \\
\hline $\mathrm{C}(29)-\mathrm{C}(30)-\mathrm{C}(31)$ & $122.3(5)$ \\
\hline $\mathrm{C}\left(31^{\prime}\right)-\mathrm{C}(30)-\mathrm{C}\left(29^{\prime}\right)$ & $119.0(11)$ \\
\hline $\mathrm{C}(29)-\mathrm{C}(30)-\mathrm{C}\left(29^{\prime}\right)$ & $26.3(8)$ \\
\hline $\mathrm{C}(31)-\mathrm{C}(30)-\mathrm{C}\left(29^{\prime}\right)$ & $114.8(8)$ \\
\hline $\mathrm{C}\left(31^{\prime}\right)-\mathrm{C}(30)-\mathrm{Cl}(2)$ & $120.3(8)$ \\
\hline $\mathrm{C}(29)-\mathrm{C}(30)-\mathrm{Cl}(2)$ & $119.1(4)$ \\
\hline $\mathrm{C}(31)-\mathrm{C}(30)-\mathrm{Cl}(2)$ & $118.6(4)$ \\
\hline
\end{tabular}




$\begin{array}{lc}\mathrm{C}\left(29^{\prime}\right)-\mathrm{C}(30)-\mathrm{Cl}(2) & 120.6(8) \\ \mathrm{C}(32)-\mathrm{C}(31)-\mathrm{C}(30) & 117.9(6) \\ \mathrm{C}(30)-\mathrm{C}\left(31^{\prime}\right)-\mathrm{C}\left(32^{\prime}\right) & 121.2(14) \\ \mathrm{C}(31)-\mathrm{C}(32)-\mathrm{C}(27) & 120.1(6) \\ \mathrm{C}(27)-\mathrm{C}\left(32^{\prime}\right)-\mathrm{C}\left(31^{\prime}\right) & 121.8(14) \\ \mathrm{C}(34)-\mathrm{C}(33)-\mathrm{C}(38) & 118.9(3) \\ \mathrm{C}(34)-\mathrm{C}(33)-\mathrm{C}(12) & 120.3(3) \\ \mathrm{C}(38)-\mathrm{C}(33)-\mathrm{C}(12) & 120.8(3) \\ \mathrm{C}(33)-\mathrm{C}(34)-\mathrm{C}(35) & 120.9(3) \\ \mathrm{C}(36)-\mathrm{C}(35)-\mathrm{C}(34) & 118.6(3) \\ \mathrm{C}(37)-\mathrm{C}(36)-\mathrm{C}(35) & 121.7(3) \\ \mathrm{C}(37)-\mathrm{C}(36)-\mathrm{Cl}(3) & 119.8(3) \\ \mathrm{C}(35)-\mathrm{C}(36)-\mathrm{Cl}(3) & 118.4(3) \\ \mathrm{C}(36)-\mathrm{C}(37)-\mathrm{C}(38) & 119.4(3) \\ \mathrm{C}(37)-\mathrm{C}(38)-\mathrm{C}(33) & 120.4(3) \\ \mathrm{C}\left(40^{\prime}\right)-\mathrm{C}(39)-\mathrm{C}(44) & 112.2(12) \\ \mathrm{C}\left(40^{\prime}\right)-\mathrm{C}(39)-\mathrm{C}(40) & 23.8(10) \\ \mathrm{C}(44)-\mathrm{C}(39)-\mathrm{C}(40) & 117.8(5) \\ \mathrm{C}\left(40^{\prime}\right)-\mathrm{C}(39)-\mathrm{C}\left(44^{\prime}\right) & 119.2(14) \\ \mathrm{C}(44)-\mathrm{C}(39)-\mathrm{C}\left(44^{\prime}\right) & 27.3(12) \\ \mathrm{C}(40)-\mathrm{C}(39)-\mathrm{C}\left(44^{\prime}\right) & 112.5(8) \\ \mathrm{C}\left(40^{\prime}\right)-\mathrm{C}(39)-\mathrm{C}(17) & 119.0(11) \\ \mathrm{C}(44)-\mathrm{C}(39)-\mathrm{C}(17) & 121.8(4) \\ \mathrm{C}(40)-\mathrm{C}(39)-\mathrm{C}(17) & 120.2(4) \\ \mathrm{C}\left(44^{\prime}\right)-\mathrm{C}(39)-\mathrm{C}(17) & 121.8(10) \\ \mathrm{C}(41)-\mathrm{C}(40)-\mathrm{C}(39) & 121.3(7) \\ \mathrm{C}(39)-\mathrm{C}\left(400^{\prime}\right)-\mathrm{C}\left(41^{\prime}\right) & 117.2(19) \\ \mathrm{C}(40)-\mathrm{C}(41)-\mathrm{C}(42) & 118.5(6) \\ & \\ & \\ & \\ & \end{array}$

\begin{tabular}{|c|c|}
\hline $\mathrm{C}(42)-\mathrm{C}\left(41^{\prime}\right)-\mathrm{C}\left(40^{\prime}\right)$ & $122.3(16)$ \\
\hline $\mathrm{C}\left(41^{\prime}\right)-\mathrm{C}(42)-\mathrm{C}(43)$ & $112.8(9)$ \\
\hline $\mathrm{C}\left(41^{\prime}\right)-\mathrm{C}(42)-\mathrm{C}(41)$ & $28.3(10)$ \\
\hline $\mathrm{C}(43)-\mathrm{C}(42)-\mathrm{C}(41)$ & $121.5(4)$ \\
\hline $\mathrm{C}\left(41^{\prime}\right)-\mathrm{C}(42)-\mathrm{C}\left(43^{\prime}\right)$ & $121.4(11)$ \\
\hline $\mathrm{C}(43)-\mathrm{C}(42)-\mathrm{C}\left(43^{\prime}\right)$ & 27.4(9) \\
\hline $\mathrm{C}(41)-\mathrm{C}(42)-\mathrm{C}\left(43^{\prime}\right)$ & $115.2(8)$ \\
\hline $\mathrm{C}\left(41^{\prime}\right)-\mathrm{C}(42)-\mathrm{Cl}(4)$ & $120.0(8)$ \\
\hline $\mathrm{C}(43)-\mathrm{C}(42)-\mathrm{Cl}(4)$ & $119.6(3)$ \\
\hline $\mathrm{C}(41)-\mathrm{C}(42)-\mathrm{Cl}(4)$ & $118.9(3)$ \\
\hline $\mathrm{C}\left(43^{\prime}\right)-\mathrm{C}(42)-\mathrm{Cl}(4)$ & $118.6(8)$ \\
\hline $\mathrm{C}(42)-\mathrm{C}(43)-\mathrm{C}(44)$ & $120.2(6)$ \\
\hline$C\left(44^{\prime}\right)-C\left(43^{\prime}\right)-C(42)$ & $115.3(16)$ \\
\hline $\mathrm{C}(43)-\mathrm{C}(44)-\mathrm{C}(39)$ & $120.6(6)$ \\
\hline $\mathrm{C}\left(43^{\prime}\right)-\mathrm{C}\left(44^{\prime}\right)-\mathrm{C}(39)$ & $124.0(18)$ \\
\hline $\mathrm{O}(1)-\mathrm{C}(45)-\mathrm{C}(46)$ & $105.8(3)$ \\
\hline $\mathrm{C}(47)-\mathrm{C}(46)-\mathrm{C}(45)$ & $104.5(3)$ \\
\hline $\mathrm{C}(46)-\mathrm{C}(47)-\mathrm{C}(48)$ & $104.4(3)$ \\
\hline $\mathrm{O}(1)-\mathrm{C}(48)-\mathrm{C}(47)$ & $104.7(3)$ \\
\hline $\mathrm{O}(2)-\mathrm{C}(49)-\mathrm{C}(50)$ & $106.2(3)$ \\
\hline $\mathrm{C}(49)-\mathrm{C}(50)-\mathrm{C}(52)$ & $104.5(3)$ \\
\hline $\mathrm{O}(2)-\mathrm{C}(51)-\mathrm{C}(52)$ & $104.9(3)$ \\
\hline $\mathrm{C}(50)-\mathrm{C}(52)-\mathrm{C}(51)$ & $104.3(3)$ \\
\hline $\mathrm{O}(3)-\mathrm{C}(53)-\mathrm{Co}(1)$ & $177.9(3)$ \\
\hline $\mathrm{O}(4)-\mathrm{C}(54)-\mathrm{Co}(1)$ & $177.3(4)$ \\
\hline $\mathrm{O}(5)-\mathrm{C}(55)-\mathrm{Co}(1)$ & $178.4(4)$ \\
\hline $\mathrm{O}(6)-\mathrm{C}(56)-\mathrm{Co}(1)$ & $176.9(4)$ \\
\hline
\end{tabular}


Table S4. Anisotropic displacement parameters $\left(\AA^{2} \times 10^{3}\right)$ for 1. The anisotropic displacement factor exponent takes the form: $-2 \pi^{2}\left[h^{2} a^{* 2} U^{11}+\ldots+2 h k a^{*} b^{*} U^{12}\right]$

\begin{tabular}{|c|c|c|c|c|c|c|}
\hline & $\mathrm{U}^{11}$ & $\mathrm{U}^{22}$ & $\mathrm{U}^{33}$ & $U^{23}$ & $\mathrm{U}^{13}$ & $\mathrm{U}^{12}$ \\
\hline $\mathrm{Co}(1)$ & $43(1)$ & $44(1)$ & $42(1)$ & $-3(1)$ & $11(1)$ & $4(1)$ \\
\hline $\operatorname{Al}(1)$ & $15(1)$ & $19(1)$ & $15(1)$ & $1(1)$ & $5(1)$ & $0(1)$ \\
\hline $\mathrm{Cl}(1)$ & $58(1)$ & $19(1)$ & $64(1)$ & $0(1)$ & $33(1)$ & $0(1)$ \\
\hline $\mathrm{Cl}(2)$ & $64(1)$ & $57(1)$ & $39(1)$ & $4(1)$ & $40(1)$ & $4(1)$ \\
\hline $\mathrm{Cl}(3)$ & $63(1)$ & $20(1)$ & 61(1) & $2(1)$ & $28(1)$ & $5(1)$ \\
\hline $\mathrm{Cl}(4)$ & $52(1)$ & $44(1)$ & $35(1)$ & $-1(1)$ & $32(1)$ & $-1(1)$ \\
\hline $\mathrm{O}(1)$ & $19(1)$ & $30(1)$ & $17(1)$ & $4(1)$ & $6(1)$ & $-3(1)$ \\
\hline $\mathrm{O}(2)$ & $17(1)$ & $32(1)$ & $16(1)$ & $-2(1)$ & $4(1)$ & $2(1)$ \\
\hline $\mathrm{O}(3)$ & $50(2)$ & $59(2)$ & $41(2)$ & $5(1)$ & $-8(1)$ & $-8(1)$ \\
\hline $\mathrm{O}(4)$ & $113(3)$ & $40(2)$ & $80(2)$ & $4(2)$ & $61(2)$ & $8(2)$ \\
\hline $\mathrm{O}(5)$ & $107(3)$ & $79(2)$ & $57(2)$ & $29(2)$ & $18(2)$ & $9(2)$ \\
\hline $\mathrm{O}(6)$ & $48(2)$ & $95(3)$ & 99(3) & $-25(2)$ & $24(2)$ & $2(2)$ \\
\hline $\mathrm{N}(1)$ & $19(1)$ & $24(1)$ & $17(1)$ & $-2(1)$ & $7(1)$ & $-1(1)$ \\
\hline $\mathrm{N}(2)$ & $18(1)$ & 21(1) & $16(1)$ & $1(1)$ & $6(1)$ & $0(1)$ \\
\hline $\mathrm{N}(3)$ & $18(1)$ & 21(1) & $17(1)$ & $1(1)$ & $5(1)$ & $1(1)$ \\
\hline $\mathrm{N}(4)$ & $19(1)$ & $23(1)$ & $16(1)$ & $3(1)$ & $7(1)$ & $-1(1)$ \\
\hline$C(1)$ & $18(1)$ & $22(1)$ & 19(1) & $2(1)$ & $3(1)$ & $-2(1)$ \\
\hline$C(2)$ & $18(1)$ & 21(1) & 19(1) & $0(1)$ & $3(1)$ & $0(1)$ \\
\hline$C(3)$ & $18(1)$ & $20(1)$ & $18(1)$ & $0(1)$ & $3(1)$ & $1(1)$ \\
\hline$C(4)$ & $30(2)$ & $17(2)$ & $23(2)$ & $-2(1)$ & $11(1)$ & $4(1)$ \\
\hline$C(5)$ & $29(2)$ & $22(1)$ & $22(1)$ & $-2(1)$ & $12(1)$ & $3(1)$ \\
\hline$C(6)$ & $18(1)$ & $28(1)$ & $14(1)$ & $0(1)$ & $6(1)$ & $2(1)$ \\
\hline$C(7)$ & $18(1)$ & $27(1)$ & $16(1)$ & $3(1)$ & $6(1)$ & $1(1)$ \\
\hline$C(8)$ & $19(1)$ & $26(1)$ & $16(1)$ & $3(1)$ & $6(1)$ & $-1(1)$ \\
\hline$C(9)$ & $29(2)$ & $26(2)$ & $25(2)$ & $2(1)$ & $13(1)$ & $-2(1)$ \\
\hline$C(10)$ & $31(2)$ & $19(2)$ & $25(2)$ & $5(1)$ & $10(1)$ & $1(1)$ \\
\hline $\mathrm{C}(11)$ & $17(1)$ & $20(1)$ & $20(1)$ & $1(1)$ & $2(1)$ & $0(1)$ \\
\hline$C(12)$ & $17(1)$ & $22(1)$ & $18(1)$ & $1(1)$ & $3(1)$ & $0(1)$ \\
\hline$C(13)$ & $16(1)$ & $26(1)$ & $17(1)$ & $-1(1)$ & $2(1)$ & $0(1)$ \\
\hline$C(14)$ & $30(2)$ & $23(2)$ & $24(2)$ & $-4(1)$ & $8(1)$ & $3(1)$ \\
\hline
\end{tabular}




\begin{tabular}{|c|c|c|c|c|c|c|}
\hline$C(15)$ & $26(2)$ & $27(2)$ & $21(1)$ & $-3(1)$ & $9(1)$ & $2(1)$ \\
\hline$C(16)$ & $18(1)$ & $25(1)$ & $16(1)$ & $-2(1)$ & $4(1)$ & $1(1)$ \\
\hline $\mathrm{C}(17)$ & $19(1)$ & $27(1)$ & $16(1)$ & $0(1)$ & $7(1)$ & $-2(1)$ \\
\hline $\mathrm{C}(18)$ & $19(1)$ & $30(2)$ & $16(1)$ & $1(1)$ & $7(1)$ & $-3(1)$ \\
\hline$C(19)$ & $30(2)$ & $26(2)$ & $25(1)$ & $4(1)$ & $14(1)$ & $-2(1)$ \\
\hline $\mathrm{C}(20)$ & $30(2)$ & $22(2)$ & $26(2)$ & $2(1)$ & $14(1)$ & $-5(1)$ \\
\hline $\mathrm{C}(21)$ & $25(2)$ & $19(1)$ & $25(1)$ & $2(1)$ & $13(1)$ & $0(1)$ \\
\hline $\mathrm{C}(22)$ & $32(2)$ & $28(2)$ & $43(2)$ & $5(1)$ & $-6(2)$ & $-4(1)$ \\
\hline$C(23)$ & $38(2)$ & $26(2)$ & $55(2)$ & $7(2)$ & $0(2)$ & $5(1)$ \\
\hline $\mathrm{C}(24)$ & $33(2)$ & $19(1)$ & $33(2)$ & $2(1)$ & $23(1)$ & $1(1)$ \\
\hline $\mathrm{C}(25)$ & $38(2)$ & $26(2)$ & $46(2)$ & $-8(1)$ & $3(2)$ & $-6(1)$ \\
\hline$C(26)$ & $31(2)$ & $28(2)$ & $33(2)$ & $-1(1)$ & $-2(1)$ & $0(1)$ \\
\hline $\mathrm{C}(27)$ & $25(2)$ & $21(1)$ & $19(1)$ & $2(1)$ & $12(1)$ & $2(1)$ \\
\hline $\mathrm{C}(28)$ & $32(3)$ & $46(5)$ & $22(3)$ & $-3(4)$ & $12(2)$ & $-12(3)$ \\
\hline $\mathrm{C}\left(28^{\prime}\right)$ & $27(6)$ & $40(11)$ & $29(7)$ & $6(9)$ & $12(5)$ & $3(8)$ \\
\hline $\mathrm{C}(29)$ & $48(4)$ & $40(4)$ & $25(3)$ & $-5(3)$ & $15(2)$ & $-16(3)$ \\
\hline$C\left(29^{\prime}\right)$ & $34(5)$ & $45(11)$ & $14(6)$ & $-2(8)$ & $10(4)$ & $-3(8)$ \\
\hline $\mathrm{C}(30)$ & $40(2)$ & $29(2)$ & $24(2)$ & $7(1)$ & $22(1)$ & $6(1)$ \\
\hline $\mathrm{C}(31)$ & $23(3)$ & $56(5)$ & $37(3)$ & $10(3)$ & $14(2)$ & $-2(3)$ \\
\hline$C\left(31^{\prime}\right)$ & $22(6)$ & $45(10)$ & $24(5)$ & $16(7)$ & $12(4)$ & $-2(7)$ \\
\hline $\mathrm{C}(32)$ & $26(3)$ & $52(5)$ & $25(3)$ & $5(3)$ & $5(2)$ & $-2(3)$ \\
\hline$C\left(32^{\prime}\right)$ & $17(6)$ & $56(12)$ & $19(6)$ & $19(8)$ & $5(4)$ & $5(8)$ \\
\hline $\mathrm{C}(33)$ & $27(2)$ & $21(1)$ & $18(1)$ & $-2(1)$ & $7(1)$ & $-1(1)$ \\
\hline $\mathrm{C}(34)$ & $26(2)$ & $26(2)$ & $28(2)$ & $2(1)$ & $7(1)$ & $3(1)$ \\
\hline $\mathrm{C}(35)$ & $33(2)$ & $26(2)$ & $30(2)$ & $-2(1)$ & $10(1)$ & $-4(1)$ \\
\hline $\mathrm{C}(36)$ & $44(2)$ & $21(2)$ & $33(2)$ & $0(1)$ & $18(2)$ & $4(1)$ \\
\hline $\mathrm{C}(37)$ & $28(2)$ & $29(2)$ & $32(2)$ & $4(1)$ & $5(1)$ & $10(1)$ \\
\hline $\mathrm{C}(38)$ & $22(2)$ & $29(2)$ & $29(2)$ & $-1(1)$ & $4(1)$ & $1(1)$ \\
\hline$C(39)$ & $23(1)$ & $23(1)$ & $19(1)$ & $-1(1)$ & $8(1)$ & $-1(1)$ \\
\hline $\mathrm{C}(40)$ & $26(3)$ & $43(4)$ & $27(3)$ & $-8(4)$ & $11(2)$ & $-1(3)$ \\
\hline$C\left(40^{\prime}\right)$ & $26(7)$ & $51(13)$ & $12(7)$ & $-4(10)$ & $9(5)$ & $13(10)$ \\
\hline $\mathrm{C}(41)$ & $29(2)$ & $46(4)$ & $20(2)$ & 1(3) & $5(2)$ & $-4(3)$ \\
\hline$C\left(41^{\prime}\right)$ & $43(7)$ & $42(11)$ & $15(6)$ & $6(8)$ & $18(5)$ & $5(8)$ \\
\hline $\mathrm{C}(42)$ & $34(2)$ & $27(2)$ & $23(1)$ & $-2(1)$ & $18(1)$ & $-2(1)$ \\
\hline$C(43)$ & $26(3)$ & $45(4)$ & $28(3)$ & $-5(3)$ & $12(2)$ & $-11(3)$ \\
\hline$C\left(43^{\prime}\right)$ & $20(6)$ & $60(13)$ & $33(7)$ & 1(9) & $14(4)$ & $-9(8)$ \\
\hline
\end{tabular}




\begin{tabular}{lllllll}
$\mathrm{C}(44)$ & $30(3)$ & $53(4)$ & $27(3)$ & $-17(3)$ & $14(2)$ & $-12(3)$ \\
$\mathrm{C}(44 ')$ & $18(7)$ & $81(15)$ & $15(6)$ & $-28(9)$ & $3(5)$ & $1(10)$ \\
$\mathrm{C}(45)$ & $21(2)$ & $50(2)$ & $34(2)$ & $12(2)$ & $8(1)$ & $-6(1)$ \\
$\mathrm{C}(46)$ & $24(2)$ & $42(2)$ & $43(2)$ & $10(2)$ & $-2(1)$ & $-4(2)$ \\
$\mathrm{C}(47)$ & $35(2)$ & $47(2)$ & $31(2)$ & $1(2)$ & $-5(1)$ & $-9(2)$ \\
$\mathrm{C}(48)$ & $29(2)$ & $37(2)$ & $17(1)$ & $4(1)$ & $4(1)$ & $0(1)$ \\
$\mathrm{C}(49)$ & $24(2)$ & $121(4)$ & $38(2)$ & $-16(2)$ & $8(2)$ & $4(2)$ \\
$\mathrm{C}(50)$ & $22(2)$ & $126(4)$ & $43(2)$ & $-20(2)$ & $2(2)$ & $-2(2)$ \\
$\mathrm{C}(51)$ & $37(2)$ & $73(3)$ & $22(2)$ & $-7(2)$ & $5(1)$ & $1(2)$ \\
$\mathrm{C}(52)$ & $38(2)$ & $68(2)$ & $31(2)$ & $0(2)$ & $-6(2)$ & $7(2)$ \\
$\mathrm{C}(53)$ & $41(2)$ & $40(2)$ & $60(3)$ & $16(2)$ & $18(2)$ & $7(2)$ \\
$\mathrm{C}(54)$ & $62(3)$ & $49(2)$ & $45(2)$ & $6(2)$ & $25(2)$ & $-8(2)$ \\
$\mathrm{C}(55)$ & $51(2)$ & $58(3)$ & $56(3)$ & $-14(2)$ & $8(2)$ & $11(2)$ \\
$\mathrm{C}(56)$ & $49(3)$ & $64(3)$ & $44(2)$ & $-14(2)$ & $7(2)$ & $5(2)$ \\
& & & & & & \\
\hline
\end{tabular}


Table S5. Hydrogen coordinates ( x 10 $\left.{ }^{4}\right)$ and isotropic displacement parameters $\left(\AA^{2} \times 10^{3}\right)$ for $\mathbf{1}$.

\begin{tabular}{|c|c|c|c|c|}
\hline & $\mathrm{x}$ & $\mathrm{y}$ & $\mathrm{z}$ & $\mathrm{U}(\mathrm{eq})$ \\
\hline $\mathrm{H}(4)$ & $1040(30)$ & $-2718(19)$ & $1537(16)$ & $18(8)$ \\
\hline $\mathrm{H}(5)$ & $1830(30)$ & $-1993(14)$ & $877(18)$ & $23(8)$ \\
\hline $\mathrm{H}(9)$ & $1660(30)$ & $308(17)$ & $830(20)$ & $33(9)$ \\
\hline $\mathrm{H}(10)$ & $900(30)$ & $1050(20)$ & $1470(20)$ & $36(10)$ \\
\hline $\mathrm{H}(14)$ & $-1110(30)$ & 1098(19) & $3543(19)$ & $35(9)$ \\
\hline $\mathrm{H}(15)$ & $-1800(30)$ & $322(17)$ & $4200(20)$ & $34(9)$ \\
\hline $\mathrm{H}(19 \mathrm{~A})$ & -1788 & -2020 & 4187 & 31 \\
\hline $\mathrm{H}(20)$ & $-980(30)$ & $-2700(20)$ & $3510(20)$ & $35(11)$ \\
\hline $\mathrm{H}(22 \mathrm{~A})$ & 1511 & -3126 & 3225 & 44 \\
\hline $\mathrm{H}(23 \mathrm{~A})$ & 1502 & -4155 & 3226 & 49 \\
\hline $\mathrm{H}(25 \mathrm{~A})$ & -1462 & -4153 & 1845 & 45 \\
\hline $\mathrm{H}(26 \mathrm{~A})$ & -1463 & -3127 & 1842 & 39 \\
\hline $\mathrm{H}(28 \mathrm{~A})$ & 667 & -1120 & -48 & 39 \\
\hline $\mathrm{H}(28 \mathrm{~B})$ & 542 & -677 & -90 & 37 \\
\hline $\mathrm{H}(29 \mathrm{~A})$ & 1613 & -1157 & -932 & 44 \\
\hline $\mathrm{H}(29 \mathrm{~B})$ & 1487 & -688 & -964 & 37 \\
\hline $\mathrm{H}(31 \mathrm{~A})$ & 4508 & -546 & 292 & 45 \\
\hline $\mathrm{H}(31 \mathrm{~B})$ & 4463 & -1062 & 290 & 35 \\
\hline $\mathrm{H}(32 \mathrm{~A})$ & 3547 & -512 & 1184 & 41 \\
\hline $\mathrm{H}(32 \mathrm{~B})$ & 3586 & -992 & 1182 & 37 \\
\hline $\mathrm{H}(34)$ & $1590(40)$ & $1434(17)$ & 2793(18) & $31(9)$ \\
\hline $\mathrm{H}(35)$ & $1660(30)$ & $2459(15)$ & $2764(16)$ & $20(8)$ \\
\hline $\mathrm{H}(37)$ & $-1760(40)$ & $2443(16)$ & $2195(18)$ & $27(8)$ \\
\hline $\mathrm{H}(38)$ & $-1750(40)$ & $1442(19)$ & $2200(20)$ & $41(10)$ \\
\hline $\mathrm{H}(40 \mathrm{~A})$ & -591 & -639 & 5121 & 37 \\
\hline $\mathrm{H}(40 \mathrm{~B})$ & -572 & -1040 & 5100 & 34 \\
\hline $\mathrm{H}(41 \mathrm{~A})$ & -1461 & -680 & 6034 & 38 \\
\hline $\mathrm{H}(41 \mathrm{~B})$ & -1607 & -1195 & 5986 & 38 \\
\hline $\mathrm{H}(43 \mathrm{~A})$ & -4454 & -1145 & 4812 & 39 \\
\hline $\mathrm{H}(43 \mathrm{~B})$ & -4540 & -653 & 4762 & 44 \\
\hline $\mathrm{H}(44 \mathrm{~A})$ & -3585 & -1137 & 3899 & 43 \\
\hline
\end{tabular}




\begin{tabular}{lllll}
$\mathrm{H}(44 \mathrm{~B})$ & -3604 & -644 & 3889 & 45 \\
$\mathrm{H}(45 \mathrm{~A})$ & 2577 & -229 & 2911 & 42 \\
$\mathrm{H}(45 \mathrm{~B})$ & 2766 & -895 & 2681 & 42 \\
$\mathrm{H}(46 \mathrm{~B})$ & $3640(30)$ & $-1090(19)$ & $3633(17)$ & $22(9)$ \\
$\mathrm{H}(46 \mathrm{~A})$ & $4120(30)$ & $-433(16)$ & $3722(18)$ & $32(8)$ \\
$\mathrm{H}(47 \mathrm{~A})$ & 2736 & -299 & 4368 & 47 \\
$\mathrm{H}(47 \mathrm{~B})$ & 3172 & -950 & 4618 & 47 \\
$\mathrm{H}(48 \mathrm{~B})$ & $1530(30)$ & $-1360(19)$ & $3927(17)$ & $28(8)$ \\
$\mathrm{H}(48 \mathrm{~A})$ & $1030(40)$ & $-733(18)$ & $4050(20)$ & $38(9)$ \\
$\mathrm{H}(49 \mathrm{~A})$ & -2644 & -303 & 2164 & 73 \\
$\mathrm{H}(49 \mathrm{~B})$ & -2725 & -974 & 2408 & 73 \\
$\mathrm{H}(50 \mathrm{~A})$ & -4128 & -576 & 1374 & 77 \\
$\mathrm{H}(50 \mathrm{~B})$ & -3754 & -1261 & 1425 & 77 \\
$\mathrm{H}(51 \mathrm{~A})$ & -1554 & -1383 & 1069 & 53 \\
$\mathrm{H}(51 \mathrm{~B})$ & -1097 & -728 & 967 & 53 \\
$\mathrm{H}(52 \mathrm{~A})$ & -2872 & -346 & 730 & 57 \\
$\mathrm{H}(52 \mathrm{~B})$ & -3200 & -1009 & 475 & 57 \\
& & & & \\
\hline
\end{tabular}




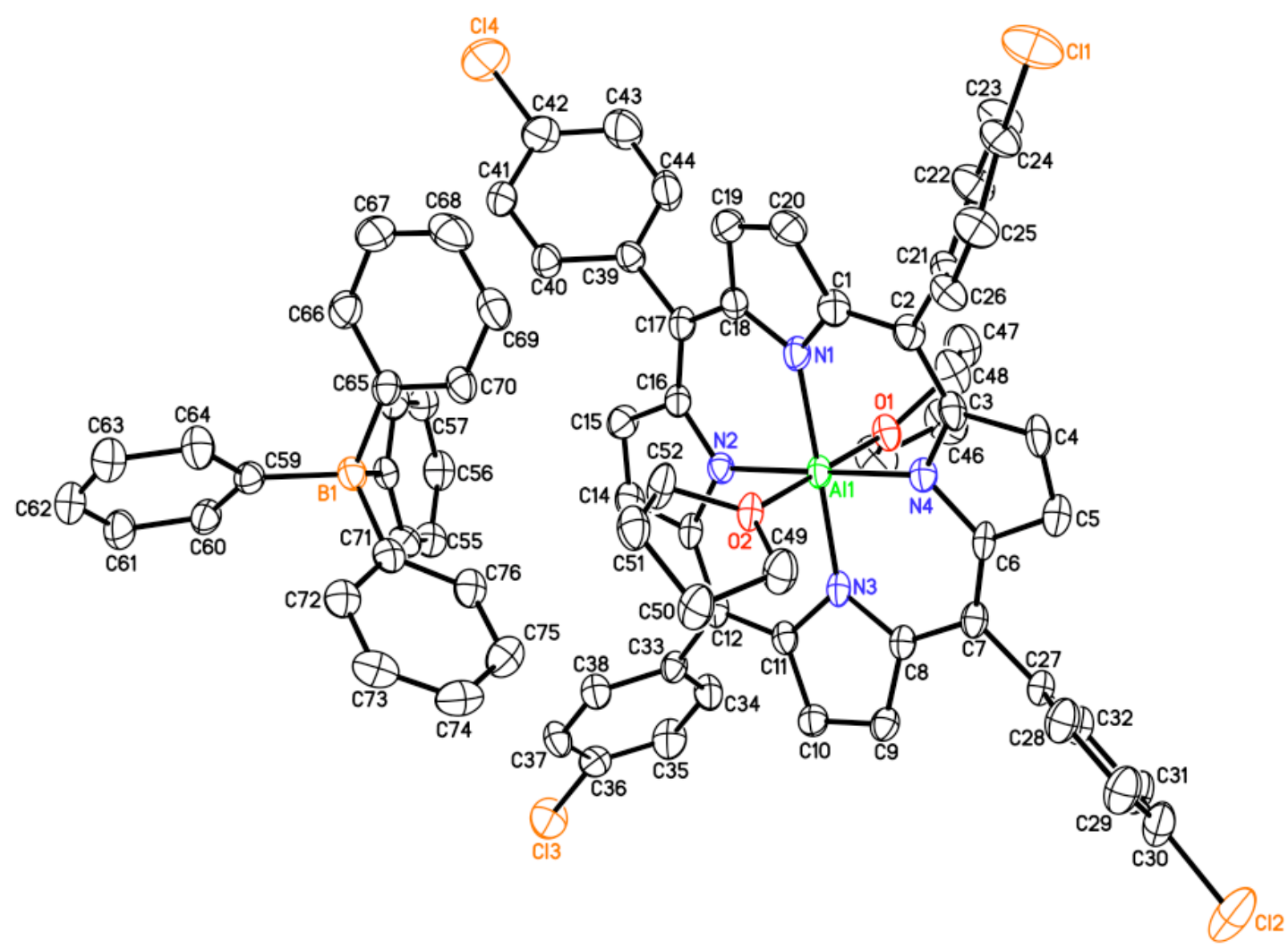

Figure S28. A view of the molecular structure of $\left[(\mathrm{ClTPP}) \mathrm{Al}(\mathrm{THF})_{2}\right]^{+}\left[\mathrm{BPh}_{4}\right]^{-}$, (hydrogen atoms omitted for clarity). Displacement ellipsoids are drawn at the $40 \%$ probability level. 
Table S6. Crystal data and structure refinement for $\left[(\mathrm{ClTPP}) \mathrm{Al}(\mathrm{THF})_{2}\right]^{+}\left[\mathrm{BPh}_{4}\right]^{-}$.

Identification code

Empirical formula

Formula weight

Temperature

Wavelength

Crystal system

Space group

Unit cell dimensions

Volume

Z

Density (calculated)

Absorption coefficient

$\mathrm{F}(000)$

Crystal size

Theta range for data collection

Index ranges

Reflections collected

Independent reflections

Completeness to theta $=22.04^{\circ}$

Absorption correction

Max. and min. transmission

Refinement method

Data / restraints / parameters

Goodness-of-fit on $\mathrm{F}^{2}$

Final $\mathrm{R}$ indices [I $>2 \operatorname{sigma}(\mathrm{I})]$

$\mathrm{R}$ indices (all data)

Largest diff. peak and hole jr7

C76 H60 Al B Cl4 N4 O2

1240.87

173(2) K

$0.71073 \AA$

Orthorhombic

Pbca

$\mathrm{a}=24.951(3) \AA$

$\alpha=90^{\circ}$

$\mathrm{b}=24.253(3) \AA$

$\beta=90^{\circ}$

$\mathrm{c}=25.448(3) \AA$

$\gamma=90^{\circ}$
15400(3) $\AA^{3}$

8

$1.070 \mathrm{Mg} / \mathrm{m}^{3}$

$0.208 \mathrm{~mm}^{-1}$

5168

$0.30 \times 0.20 \times 0.10 \mathrm{~mm}^{3}$

1.42 to $22.04^{\circ}$.

$-24<=\mathrm{h}<=26,-25<=\mathrm{k}<=23,-26<=1<=26$

48624

$9033[\mathrm{R}($ int $)=0.0522]$

$95.1 \%$

Semi-empirical from equivalents

0.9795 and 0.9403

Full-matrix least-squares on $\mathrm{F}^{2}$

9033 / 0 / 793

1.023

$\mathrm{R} 1=0.0441, \mathrm{wR} 2=0.1101$

$\mathrm{R} 1=0.0679, \mathrm{wR} 2=0.1168$

0.190 and -0.248 e. $\AA^{-3}$ 
Table S7. Atomic coordinates ( x $10^{4}$ ) and equivalent isotropic displacement parameters $\left(\AA^{2} \times 10^{3}\right)$ for $\left[(\mathrm{ClTPP}) \mathrm{Al}(\mathrm{THF})_{2}\right]^{+}\left[\mathrm{BPh}_{4}\right]^{-}$. U(eq) is defined as one third of the trace of the orthogonalized $\mathrm{U}^{\mathrm{ij}}$ tensor.

\begin{tabular}{|c|c|c|c|c|}
\hline & $\mathrm{x}$ & $\mathrm{y}$ & $\mathrm{z}$ & $\mathrm{U}(\mathrm{eq})$ \\
\hline $\mathrm{Cl}(1)$ & $6761(1)$ & $6723(1)$ & $3116(1)$ & $64(1)$ \\
\hline $\mathrm{Cl}(2)$ & $3238(1)$ & $2364(1)$ & 3903(1) & $83(1)$ \\
\hline $\mathrm{Cl}(3)$ & $4678(1)$ & $575(1)$ & $-801(1)$ & $61(1)$ \\
\hline $\mathrm{Cl}(4)$ & $8849(1)$ & $4056(1)$ & $-742(1)$ & $79(1)$ \\
\hline $\operatorname{Al}(1)$ & $5916(1)$ & $3362(1)$ & 1481(1) & $29(1)$ \\
\hline $\mathrm{O}(1)$ & $6435(1)$ & $2879(1)$ & $1835(1)$ & $35(1)$ \\
\hline $\mathrm{O}(2)$ & $5400(1)$ & $3856(1)$ & 1144(1) & $31(1)$ \\
\hline $\mathrm{N}(1)$ & $6468(1)$ & 3949(1) & 1412(1) & $33(1)$ \\
\hline $\mathrm{N}(2)$ & $6136(1)$ & $3055(1)$ & 791(1) & $30(1)$ \\
\hline $\mathrm{N}(3)$ & $5350(1)$ & 2792(1) & 1536(1) & $29(1)$ \\
\hline $\mathrm{N}(4)$ & $5697(1)$ & $3671(1)$ & $2173(1)$ & $30(1)$ \\
\hline $\mathrm{C}(1)$ & $6513(1)$ & $4422(1)$ & 1711(1) & $35(1)$ \\
\hline$C(2)$ & $6249(1)$ & $4516(1)$ & $2184(1)$ & $34(1)$ \\
\hline$C(3)$ & $5883(1)$ & $4149(1)$ & $2406(1)$ & $32(1)$ \\
\hline$C(4)$ & $5648(1)$ & $4210(1)$ & $2918(1)$ & $37(1)$ \\
\hline$C(5)$ & $5320(1)$ & $3782(1)$ & 2991(1) & $37(1)$ \\
\hline$C(6)$ & $5329(1)$ & $3451(1)$ & $2524(1)$ & $30(1)$ \\
\hline$C(7)$ & 4993(1) & $3008(1)$ & $2419(1)$ & $29(1)$ \\
\hline$C(8)$ & 4981(1) & $2728(1)$ & $1942(1)$ & $28(1)$ \\
\hline $\mathrm{C}(9)$ & 4576(1) & $2350(1)$ & 1789(1) & $34(1)$ \\
\hline$C(10)$ & $4692(1)$ & $2175(1)$ & $1297(1)$ & $34(1)$ \\
\hline $\mathrm{C}(11)$ & $5174(1)$ & 2441(1) & 1141(1) & $29(1)$ \\
\hline$C(12)$ & $5439(1)$ & $2347(1)$ & $671(1)$ & $30(1)$ \\
\hline$C(13)$ & 5903(1) & $2618(1)$ & $523(1)$ & $31(1)$ \\
\hline$C(14)$ & $6224(1)$ & $2479(1)$ & $72(1)$ & $39(1)$ \\
\hline$C(15)$ & $6649(1)$ & $2819(1)$ & $69(1)$ & $39(1)$ \\
\hline$C(16)$ & $6595(1)$ & 3183(1) & $508(1)$ & $32(1)$ \\
\hline$C(17)$ & $6925(1)$ & $3627(1)$ & $606(1)$ & $32(1)$ \\
\hline$C(18)$ & $6834(1)$ & $4007(1)$ & 1013(1) & $33(1)$ \\
\hline$C(19)$ & $7089(1)$ & $4531(1)$ & $1055(1)$ & $41(1)$ \\
\hline$C(20)$ & $6888(1)$ & $4789(1)$ & $1475(1)$ & $43(1)$ \\
\hline
\end{tabular}




\begin{tabular}{|c|c|c|c|c|}
\hline$C(21)$ & $6364(1)$ & $5047(1)$ & $2459(1)$ & $33(1)$ \\
\hline$C(22)$ & $6874(1)$ & $5172(1)$ & $2630(1)$ & $43(1)$ \\
\hline$C(23)$ & $6995(1)$ & $5681(1)$ & 2841(1) & $46(1)$ \\
\hline$C(24)$ & $6602(1)$ & $6069(1)$ & $2880(1)$ & $40(1)$ \\
\hline$C(25)$ & $6084(1)$ & $5953(1)$ & $2735(1)$ & $42(1)$ \\
\hline$C(26)$ & $5969(1)$ & $5443(1)$ & $2525(1)$ & $38(1)$ \\
\hline$C(27)$ & $4576(1)$ & $2857(1)$ & 2813(1) & $31(1)$ \\
\hline $\mathrm{C}(28)$ & $4192(1)$ & $3236(1)$ & $2970(1)$ & $45(1)$ \\
\hline$C(29)$ & $3785(1)$ & $3087(2)$ & $3309(1)$ & $53(1)$ \\
\hline$C(30)$ & $3763(1)$ & $2556(2)$ & $3492(1)$ & $51(1)$ \\
\hline $\mathrm{C}(31)$ & $4132(1)$ & 2171(1) & $3349(1)$ & $46(1)$ \\
\hline$C(32)$ & $4537(1)$ & $2326(1)$ & $3006(1)$ & $38(1)$ \\
\hline $\mathrm{C}(33)$ & $5233(1)$ & 1907(1) & $312(1)$ & $32(1)$ \\
\hline$C(34)$ & $5274(1)$ & $1358(1)$ & $446(1)$ & $41(1)$ \\
\hline$C(35)$ & $5108(1)$ & $943(1)$ & $106(1)$ & $49(1)$ \\
\hline$C(36)$ & 4893(1) & 1087(1) & $-373(1)$ & $38(1)$ \\
\hline $\mathrm{C}(37)$ & $4844(1)$ & 1631(1) & $-515(1)$ & $39(1)$ \\
\hline$C(38)$ & $5013(1)$ & $2039(1)$ & $-180(1)$ & $37(1)$ \\
\hline $\mathrm{C}(39)$ & $7398(1)$ & $3728(1)$ & $261(1)$ & $32(1)$ \\
\hline $\mathrm{C}(40)$ & $7345(1)$ & $3837(1)$ & $-270(1)$ & $35(1)$ \\
\hline $\mathrm{C}(41)$ & 7791(1) & $3935(1)$ & $-578(1)$ & $44(1)$ \\
\hline $\mathrm{C}(42)$ & $8294(1)$ & $3928(1)$ & $-354(1)$ & $48(1)$ \\
\hline $\mathrm{C}(43)$ & $8353(1)$ & $3822(2)$ & $175(1)$ & $59(1)$ \\
\hline $\mathrm{C}(44)$ & $7904(1)$ & $3725(1)$ & $476(1)$ & $50(1)$ \\
\hline $\mathrm{C}(45)$ & $6556(2)$ & $2307(1)$ & 1691(1) & $61(1)$ \\
\hline$C(46)$ & $6915(2)$ & $2105(2)$ & $2130(1)$ & $66(1)$ \\
\hline $\mathrm{C}(47)$ & $7208(1)$ & $2616(2)$ & 2292(1) & $60(1)$ \\
\hline $\mathrm{C}(48)$ & $6775(1)$ & $3046(2)$ & $2276(1)$ & $56(1)$ \\
\hline $\mathrm{C}(49)$ & 4894(1) & 4039(1) & 1371(1) & $47(1)$ \\
\hline $\mathrm{C}(50)$ & $4579(1)$ & $4254(1)$ & $919(1)$ & $46(1)$ \\
\hline $\mathrm{C}(51)$ & $5005(1)$ & $4502(1)$ & $570(1)$ & $43(1)$ \\
\hline$C(52)$ & $5472(1)$ & $4128(1)$ & $638(1)$ & $51(1)$ \\
\hline $\mathrm{B}(1)$ & $5788(1)$ & $3901(1)$ & $-1407(1)$ & $34(1)$ \\
\hline$C(53)$ & $6096(1)$ & $3300(1)$ & $-1375(1)$ & $31(1)$ \\
\hline$C(54)$ & $5830(1)$ & 2793(1) & $-1360(1)$ & $36(1)$ \\
\hline$C(55)$ & $6087(1)$ & $2292(1)$ & $-1337(1)$ & $41(1)$ \\
\hline
\end{tabular}




\begin{tabular}{lllll}
$\mathrm{C}(56)$ & $6637(1)$ & $2271(1)$ & $-1327(1)$ & $43(1)$ \\
$\mathrm{C}(57)$ & $6922(1)$ & $2755(1)$ & $-1348(1)$ & $41(1)$ \\
$\mathrm{C}(58)$ & $6653(1)$ & $3255(1)$ & $-1377(1)$ & $37(1)$ \\
$\mathrm{C}(59)$ & $5701(1)$ & $4082(1)$ & $-2023(1)$ & $34(1)$ \\
$\mathrm{C}(60)$ & $5626(1)$ & $3698(1)$ & $-2428(1)$ & $40(1)$ \\
$\mathrm{C}(61)$ & $5489(1)$ & $3847(2)$ & $-2938(1)$ & $50(1)$ \\
$\mathrm{C}(62)$ & $5417(1)$ & $4391(2)$ & $-3065(1)$ & $54(1)$ \\
$\mathrm{C}(63)$ & $5500(1)$ & $4787(2)$ & $-2685(1)$ & $51(1)$ \\
$\mathrm{C}(64)$ & $5642(1)$ & $4633(1)$ & $-2177(1)$ & $45(1)$ \\
$\mathrm{C}(65)$ & $6149(1)$ & $4361(1)$ & $-1092(1)$ & $32(1)$ \\
$\mathrm{C}(66)$ & $6588(1)$ & $4624(1)$ & $-1328(1)$ & $42(1)$ \\
$\mathrm{C}(67)$ & $6910(1)$ & $4996(1)$ & $-1062(1)$ & $50(1)$ \\
$\mathrm{C}(68)$ & $6797(2)$ & $5127(1)$ & $-544(2)$ & $56(1)$ \\
$\mathrm{C}(69)$ & $6371(1)$ & $4885(1)$ & $-297(1)$ & $46(1)$ \\
$\mathrm{C}(70)$ & $6057(1)$ & $4507(1)$ & $-569(1)$ & $37(1)$ \\
$\mathrm{C}(71)$ & $5198(1)$ & $3868(1)$ & $-1121(1)$ & $33(1)$ \\
$\mathrm{C}(72)$ & $4764(1)$ & $4176(1)$ & $-1294(1)$ & $40(1)$ \\
$\mathrm{C}(73)$ & $4274(1)$ & $4183(1)$ & $-1036(1)$ & $49(1)$ \\
$\mathrm{C}(74)$ & $4199(1)$ & $3869(1)$ & $-587(1)$ & $50(1)$ \\
$\mathrm{C}(75)$ & $4612(1)$ & $3549(1)$ & $-413(1)$ & $45(1)$ \\
$\mathrm{C}(76)$ & $5099(1)$ & $3554(1)$ & $-670(1)$ & $38(1)$ \\
\hline
\end{tabular}


Table S8. Bond lengths $[\AA]$ and angles $\left[{ }^{\circ}\right]$ for $\left[(\mathrm{ClTPP}) \mathrm{Al}(\mathrm{THF})_{2}\right]^{+}\left[\mathrm{BPh}_{4}\right]^{-}$.

\begin{tabular}{|c|c|c|c|}
\hline $\mathrm{Cl}(1)-\mathrm{C}(24)$ & $1.743(3)$ & $\mathrm{C}(10)-\mathrm{C}(11)$ & $1.422(4)$ \\
\hline $\mathrm{Cl}(2)-\mathrm{C}(30)$ & $1.740(3)$ & $\mathrm{C}(11)-\mathrm{C}(12)$ & $1.383(4)$ \\
\hline $\mathrm{Cl}(3)-\mathrm{C}(36)$ & $1.737(3)$ & $\mathrm{C}(12)-\mathrm{C}(13)$ & $1.384(4)$ \\
\hline $\mathrm{Cl}(4)-\mathrm{C}(42)$ & $1.729(3)$ & $\mathrm{C}(12)-\mathrm{C}(33)$ & $1.498(4)$ \\
\hline $\mathrm{Al}(1)-\mathrm{O}(2)$ & $1.9571(19)$ & $\mathrm{C}(13)-\mathrm{C}(14)$ & $1.437(4)$ \\
\hline $\mathrm{Al}(1)-\mathrm{O}(1)$ & $1.965(2)$ & $\mathrm{C}(14)-\mathrm{C}(15)$ & $1.343(4)$ \\
\hline $\mathrm{Al}(1)-\mathrm{N}(3)$ & $1.980(2)$ & $\mathrm{C}(15)-\mathrm{C}(16)$ & $1.430(4)$ \\
\hline $\mathrm{Al}(1)-\mathrm{N}(2)$ & $1.986(2)$ & $\mathrm{C}(16)-\mathrm{C}(17)$ & $1.379(4)$ \\
\hline $\mathrm{Al}(1)-\mathrm{N}(1)$ & $1.988(2)$ & $\mathrm{C}(17)-\mathrm{C}(18)$ & $1.405(4)$ \\
\hline $\mathrm{Al}(1)-\mathrm{N}(4)$ & $1.990(2)$ & $\mathrm{C}(17)-\mathrm{C}(39)$ & $1.491(4)$ \\
\hline $\mathrm{O}(1)-\mathrm{C}(45)$ & $1.466(4)$ & $\mathrm{C}(18)-\mathrm{C}(19)$ & $1.425(4)$ \\
\hline $\mathrm{O}(1)-\mathrm{C}(48)$ & $1.465(3)$ & $\mathrm{C}(19)-\mathrm{C}(20)$ & $1.337(4)$ \\
\hline $\mathrm{O}(2)-\mathrm{C}(52)$ & $1.458(3)$ & $\mathrm{C}(21)-\mathrm{C}(22)$ & $1.379(4)$ \\
\hline $\mathrm{O}(2)-\mathrm{C}(49)$ & $1.458(3)$ & $\mathrm{C}(21)-\mathrm{C}(26)$ & $1.387(4)$ \\
\hline $\mathrm{N}(1)-\mathrm{C}(18)$ & $1.373(3)$ & $\mathrm{C}(22)-\mathrm{C}(23)$ & $1.380(4)$ \\
\hline $\mathrm{N}(1)-\mathrm{C}(1)$ & $1.382(3)$ & $\mathrm{C}(23)-\mathrm{C}(24)$ & $1.364(4)$ \\
\hline $\mathrm{N}(2)-\mathrm{C}(16)$ & $1.387(3)$ & $\mathrm{C}(24)-\mathrm{C}(25)$ & $1.371(4)$ \\
\hline $\mathrm{N}(2)-\mathrm{C}(13)$ & $1.388(3)$ & $\mathrm{C}(25)-\mathrm{C}(26)$ & $1.379(4)$ \\
\hline $\mathrm{N}(3)-\mathrm{C}(11)$ & $1.389(3)$ & $\mathrm{C}(27)-\mathrm{C}(32)$ & $1.382(4)$ \\
\hline $\mathrm{N}(3)-\mathrm{C}(8)$ & $1.393(3)$ & $\mathrm{C}(27)-\mathrm{C}(28)$ & $1.386(4)$ \\
\hline $\mathrm{N}(4)-\mathrm{C}(3)$ & $1.382(3)$ & $\mathrm{C}(28)-\mathrm{C}(29)$ & $1.379(4)$ \\
\hline $\mathrm{N}(4)-\mathrm{C}(6)$ & $1.389(3)$ & $\mathrm{C}(29)-\mathrm{C}(30)$ & $1.371(4)$ \\
\hline $\mathrm{C}(1)-\mathrm{C}(2)$ & $1.389(4)$ & $\mathrm{C}(30)-\mathrm{C}(31)$ & $1.362(4)$ \\
\hline$C(1)-C(20)$ & $1.425(4)$ & $\mathrm{C}(31)-\mathrm{C}(32)$ & $1.387(4)$ \\
\hline $\mathrm{C}(2)-\mathrm{C}(3)$ & $1.395(4)$ & $\mathrm{C}(33)-\mathrm{C}(34)$ & $1.377(4)$ \\
\hline$C(2)-C(21)$ & $1.495(4)$ & $\mathrm{C}(33)-\mathrm{C}(38)$ & $1.402(4)$ \\
\hline $\mathrm{C}(3)-\mathrm{C}(4)$ & $1.436(4)$ & $\mathrm{C}(34)-\mathrm{C}(35)$ & $1.390(4)$ \\
\hline$C(4)-C(5)$ & $1.334(4)$ & $\mathrm{C}(35)-\mathrm{C}(36)$ & $1.378(4)$ \\
\hline$C(5)-C(6)$ & $1.433(4)$ & $\mathrm{C}(36)-\mathrm{C}(37)$ & $1.374(4)$ \\
\hline$C(6)-C(7)$ & $1.388(4)$ & $\mathrm{C}(37)-\mathrm{C}(38)$ & $1.372(4)$ \\
\hline$C(7)-C(8)$ & $1.393(4)$ & C(39)-C(44) & $1.375(4)$ \\
\hline$C(7)-C(27)$ & $1.492(4)$ & C(39)-C(40) & $1.383(4)$ \\
\hline$C(8)-C(9)$ & $1.417(4)$ & $\mathrm{C}(40)-\mathrm{C}(41)$ & $1.384(4)$ \\
\hline $\mathrm{C}(9)-\mathrm{C}(10)$ & $1.352(4)$ & $\mathrm{C}(41)-\mathrm{C}(42)$ & $1.379(4)$ \\
\hline
\end{tabular}




\begin{tabular}{|c|c|c|c|}
\hline$C(42)-C(43)$ & $1.377(4)$ & & \\
\hline$C(43)-C(44)$ & $1.379(4)$ & $\mathrm{O}(2)-\mathrm{Al}(1)-\mathrm{O}(1)$ & $178.56(8)$ \\
\hline$C(45)-C(46)$ & $1.515(4)$ & $\mathrm{O}(2)-\mathrm{Al}(1)-\mathrm{N}(3)$ & $89.43(9)$ \\
\hline$C(46)-C(47)$ & $1.497(5)$ & $\mathrm{O}(1)-\mathrm{Al}(1)-\mathrm{N}(3)$ & $91.18(9)$ \\
\hline $\mathrm{C}(47)-\mathrm{C}(48)$ & $1.502(4)$ & $\mathrm{O}(2)-\mathrm{Al}(1)-\mathrm{N}(2)$ & $91.34(9)$ \\
\hline$C(49)-C(50)$ & $1.488(4)$ & $\mathrm{O}(1)-\mathrm{Al}(1)-\mathrm{N}(2)$ & $89.96(9)$ \\
\hline$C(50)-C(51)$ & $1.510(4)$ & $\mathrm{N}(3)-\mathrm{Al}(1)-\mathrm{N}(2)$ & $89.84(9)$ \\
\hline $\mathrm{C}(51)-\mathrm{C}(52)$ & $1.486(4)$ & $\mathrm{O}(2)-\mathrm{Al}(1)-\mathrm{N}(1)$ & $88.75(9)$ \\
\hline $\mathrm{B}(1)-\mathrm{C}(59)$ & $1.642(4)$ & $\mathrm{O}(1)-\mathrm{Al}(1)-\mathrm{N}(1)$ & $90.65(9)$ \\
\hline $\mathrm{B}(1)-\mathrm{C}(71)$ & $1.644(4)$ & $\mathrm{N}(3)-\mathrm{Al}(1)-\mathrm{N}(1)$ & $178.15(10)$ \\
\hline $\mathrm{B}(1)-\mathrm{C}(65)$ & $1.644(4)$ & $\mathrm{N}(2)-\mathrm{Al}(1)-\mathrm{N}(1)$ & $89.88(10)$ \\
\hline $\mathrm{B}(1)-\mathrm{C}(53)$ & $1.651(4)$ & $\mathrm{O}(2)-\mathrm{Al}(1)-\mathrm{N}(4)$ & $88.71(9)$ \\
\hline $\mathrm{C}(53)-\mathrm{C}(58)$ & $1.393(4)$ & $\mathrm{O}(1)-\mathrm{Al}(1)-\mathrm{N}(4)$ & $89.98(9)$ \\
\hline $\mathrm{C}(53)-\mathrm{C}(54)$ & $1.397(4)$ & $\mathrm{N}(3)-\mathrm{Al}(1)-\mathrm{N}(4)$ & $90.31(9)$ \\
\hline $\mathrm{C}(54)-\mathrm{C}(55)$ & $1.377(4)$ & $\mathrm{N}(2)-\mathrm{Al}(1)-\mathrm{N}(4)$ & $179.84(12)$ \\
\hline$C(55)-C(56)$ & $1.372(4)$ & $\mathrm{N}(1)-\mathrm{Al}(1)-\mathrm{N}(4)$ & $89.97(10)$ \\
\hline$C(56)-C(57)$ & $1.373(4)$ & $\mathrm{C}(45)-\mathrm{O}(1)-\mathrm{C}(48)$ & $109.5(2)$ \\
\hline $\mathrm{C}(57)-\mathrm{C}(58)$ & $1.388(4)$ & $\mathrm{C}(45)-\mathrm{O}(1)-\mathrm{Al}(1)$ & $125.82(17)$ \\
\hline $\mathrm{C}(59)-\mathrm{C}(64)$ & $1.401(4)$ & $\mathrm{C}(48)-\mathrm{O}(1)-\mathrm{Al}(1)$ & $124.60(18)$ \\
\hline $\mathrm{C}(59)-\mathrm{C}(60)$ & $1.399(4)$ & $\mathrm{C}(52)-\mathrm{O}(2)-\mathrm{C}(49)$ & $108.6(2)$ \\
\hline$C(60)-C(61)$ & $1.390(4)$ & $\mathrm{C}(52)-\mathrm{O}(2)-\mathrm{Al}(1)$ & $125.75(17)$ \\
\hline$C(61)-C(62)$ & $1.372(4)$ & $\mathrm{C}(49)-\mathrm{O}(2)-\mathrm{Al}(1)$ & $125.54(15)$ \\
\hline$C(62)-C(63)$ & $1.378(4)$ & $\mathrm{C}(18)-\mathrm{N}(1)-\mathrm{C}(1)$ & $105.6(2)$ \\
\hline$C(63)-C(64)$ & $1.392(4)$ & $\mathrm{C}(18)-\mathrm{N}(1)-\mathrm{Al}(1)$ & $126.95(19)$ \\
\hline$C(65)-C(70)$ & $1.395(4)$ & $\mathrm{C}(1)-\mathrm{N}(1)-\mathrm{Al}(1)$ & $126.91(19)$ \\
\hline$C(65)-C(66)$ & $1.404(4)$ & $\mathrm{C}(16)-\mathrm{N}(2)-\mathrm{C}(13)$ & $105.1(2)$ \\
\hline$C(66)-C(67)$ & $1.384(4)$ & $\mathrm{C}(16)-\mathrm{N}(2)-\mathrm{Al}(1)$ & $127.11(18)$ \\
\hline$C(67)-C(68)$ & $1.387(4)$ & $\mathrm{C}(13)-\mathrm{N}(2)-\mathrm{Al}(1)$ & $127.29(19)$ \\
\hline $\mathrm{C}(68)-\mathrm{C}(69)$ & $1.367(4)$ & $\mathrm{C}(11)-\mathrm{N}(3)-\mathrm{C}(8)$ & $105.0(2)$ \\
\hline$C(69)-C(70)$ & $1.390(4)$ & $\mathrm{C}(11)-\mathrm{N}(3)-\mathrm{Al}(1)$ & $127.05(17)$ \\
\hline$C(71)-C(72)$ & $1.388(4)$ & $\mathrm{C}(8)-\mathrm{N}(3)-\mathrm{Al}(1)$ & $127.01(18)$ \\
\hline$C(71)-C(76)$ & $1.399(4)$ & $\mathrm{C}(3)-\mathrm{N}(4)-\mathrm{C}(6)$ & $105.6(2)$ \\
\hline$C(72)-C(73)$ & $1.388(4)$ & $\mathrm{C}(3)-\mathrm{N}(4)-\mathrm{Al}(1)$ & $127.10(18)$ \\
\hline$C(73)-C(74)$ & $1.385(4)$ & $\mathrm{C}(6)-\mathrm{N}(4)-\mathrm{Al}(1)$ & $127.30(18)$ \\
\hline$C(74)-C(75)$ & $1.364(4)$ & $\mathrm{N}(1)-\mathrm{C}(1)-\mathrm{C}(2)$ & $125.1(3)$ \\
\hline$C(75)-C(76)$ & $1.380(4)$ & $\mathrm{N}(1)-\mathrm{C}(1)-\mathrm{C}(20)$ & $109.9(3)$ \\
\hline
\end{tabular}




\begin{tabular}{|c|c|}
\hline$C(2)-C(1)-C(20)$ & $124.9(3)$ \\
\hline $\mathrm{C}(1)-\mathrm{C}(2)-\mathrm{C}(3)$ & $123.8(3)$ \\
\hline $\mathrm{C}(1)-\mathrm{C}(2)-\mathrm{C}(21)$ & $117.2(3)$ \\
\hline $\mathrm{C}(3)-\mathrm{C}(2)-\mathrm{C}(21)$ & $119.0(2)$ \\
\hline $\mathrm{N}(4)-\mathrm{C}(3)-\mathrm{C}(2)$ & $125.5(2)$ \\
\hline $\mathrm{N}(4)-\mathrm{C}(3)-\mathrm{C}(4)$ & $109.7(3)$ \\
\hline$C(2)-C(3)-C(4)$ & $124.8(3)$ \\
\hline$C(5)-C(4)-C(3)$ & $107.3(3)$ \\
\hline$C(4)-C(5)-C(6)$ & 108.1(3) \\
\hline $\mathrm{N}(4)-\mathrm{C}(6)-\mathrm{C}(7)$ & $125.0(2)$ \\
\hline $\mathrm{N}(4)-\mathrm{C}(6)-\mathrm{C}(5)$ & $109.1(2)$ \\
\hline$C(7)-C(6)-C(5)$ & $125.7(3)$ \\
\hline$C(6)-C(7)-C(8)$ & $124.0(3)$ \\
\hline$C(6)-C(7)-C(27)$ & $118.9(2)$ \\
\hline $\mathrm{C}(8)-\mathrm{C}(7)-\mathrm{C}(27)$ & $116.8(3)$ \\
\hline $\mathrm{N}(3)-\mathrm{C}(8)-\mathrm{C}(7)$ & $125.3(3)$ \\
\hline $\mathrm{N}(3)-\mathrm{C}(8)-\mathrm{C}(9)$ & $109.9(2)$ \\
\hline$C(7)-C(8)-C(9)$ & $124.8(3)$ \\
\hline $\mathrm{C}(10)-\mathrm{C}(9)-\mathrm{C}(8)$ & $107.8(3)$ \\
\hline$C(9)-C(10)-C(11)$ & $107.3(3)$ \\
\hline $\mathrm{C}(12)-\mathrm{C}(11)-\mathrm{N}(3)$ & $125.1(3)$ \\
\hline $\mathrm{C}(12)-\mathrm{C}(11)-\mathrm{C}(10)$ & $124.9(3)$ \\
\hline $\mathrm{N}(3)-\mathrm{C}(11)-\mathrm{C}(10)$ & $110.0(2)$ \\
\hline $\mathrm{C}(13)-\mathrm{C}(12)-\mathrm{C}(11)$ & $123.9(2)$ \\
\hline $\mathrm{C}(13)-\mathrm{C}(12)-\mathrm{C}(33)$ & $117.3(2)$ \\
\hline $\mathrm{C}(11)-\mathrm{C}(12)-\mathrm{C}(33)$ & $118.7(3)$ \\
\hline $\mathrm{C}(12)-\mathrm{C}(13)-\mathrm{N}(2)$ & $125.3(2)$ \\
\hline $\mathrm{C}(12)-\mathrm{C}(13)-\mathrm{C}(14)$ & $125.0(3)$ \\
\hline $\mathrm{N}(2)-\mathrm{C}(13)-\mathrm{C}(14)$ & 109.7(3) \\
\hline $\mathrm{C}(15)-\mathrm{C}(14)-\mathrm{C}(13)$ & $107.5(3)$ \\
\hline $\mathrm{C}(14)-\mathrm{C}(15)-\mathrm{C}(16)$ & $107.4(3)$ \\
\hline $\mathrm{C}(17)-\mathrm{C}(16)-\mathrm{N}(2)$ & $125.1(2)$ \\
\hline $\mathrm{C}(17)-\mathrm{C}(16)-\mathrm{C}(15)$ & $124.5(3)$ \\
\hline $\mathrm{N}(2)-\mathrm{C}(16)-\mathrm{C}(15)$ & $110.2(3)$ \\
\hline $\mathrm{C}(16)-\mathrm{C}(17)-\mathrm{C}(18)$ & $123.3(3)$ \\
\hline $\mathrm{C}(16)-\mathrm{C}(17)-\mathrm{C}(39)$ & $119.7(2)$ \\
\hline
\end{tabular}

\begin{tabular}{|c|c|}
\hline $\mathrm{C}(18)-\mathrm{C}(17)-\mathrm{C}(39)$ & $117.0(3)$ \\
\hline $\mathrm{N}(1)-\mathrm{C}(18)-\mathrm{C}(17)$ & $125.8(3)$ \\
\hline $\mathrm{N}(1)-\mathrm{C}(18)-\mathrm{C}(19)$ & $109.5(3)$ \\
\hline $\mathrm{C}(17)-\mathrm{C}(18)-\mathrm{C}(19)$ & $124.6(3)$ \\
\hline $\mathrm{C}(20)-\mathrm{C}(19)-\mathrm{C}(18)$ & 108.1(3) \\
\hline$C(19)-C(20)-C(1)$ & $106.9(3)$ \\
\hline$C(22)-C(21)-C(26)$ & $117.8(3)$ \\
\hline$C(22)-C(21)-C(2)$ & $120.9(3)$ \\
\hline$C(26)-C(21)-C(2)$ & $121.1(3)$ \\
\hline $\mathrm{C}(23)-\mathrm{C}(22)-\mathrm{C}(21)$ & $121.4(3)$ \\
\hline$C(24)-C(23)-C(22)$ & $119.2(3)$ \\
\hline $\mathrm{C}(23)-\mathrm{C}(24)-\mathrm{C}(25)$ & $121.2(3)$ \\
\hline $\mathrm{C}(23)-\mathrm{C}(24)-\mathrm{Cl}(1)$ & $119.3(3)$ \\
\hline $\mathrm{C}(25)-\mathrm{C}(24)-\mathrm{Cl}(1)$ & $119.5(2)$ \\
\hline$C(24)-C(25)-C(26)$ & $118.9(3)$ \\
\hline$C(25)-C(26)-C(21)$ & $121.3(3)$ \\
\hline $\mathrm{C}(32)-\mathrm{C}(27)-\mathrm{C}(28)$ & $117.9(3)$ \\
\hline$C(32)-C(27)-C(7)$ & 121.1(3) \\
\hline $\mathrm{C}(28)-\mathrm{C}(27)-\mathrm{C}(7)$ & $120.9(3)$ \\
\hline $\mathrm{C}(29)-\mathrm{C}(28)-\mathrm{C}(27)$ & $121.0(3)$ \\
\hline $\mathrm{C}(30)-\mathrm{C}(29)-\mathrm{C}(28)$ & $119.2(3)$ \\
\hline $\mathrm{C}(31)-\mathrm{C}(30)-\mathrm{C}(29)$ & $121.7(3)$ \\
\hline $\mathrm{C}(31)-\mathrm{C}(30)-\mathrm{Cl}(2)$ & 119.1(3) \\
\hline $\mathrm{C}(29)-\mathrm{C}(30)-\mathrm{Cl}(2)$ & 119.1(3) \\
\hline $\mathrm{C}(30)-\mathrm{C}(31)-\mathrm{C}(32)$ & $118.4(3)$ \\
\hline$C(27)-C(32)-C(31)$ & $121.8(3)$ \\
\hline $\mathrm{C}(34)-\mathrm{C}(33)-\mathrm{C}(38)$ & 118.1(3) \\
\hline$C(34)-C(33)-C(12)$ & $120.8(3)$ \\
\hline $\mathrm{C}(38)-\mathrm{C}(33)-\mathrm{C}(12)$ & 121.1(3) \\
\hline$C(33)-C(34)-C(35)$ & $121.5(3)$ \\
\hline $\mathrm{C}(36)-\mathrm{C}(35)-\mathrm{C}(34)$ & $118.9(3)$ \\
\hline$C(37)-C(36)-C(35)$ & $120.7(3)$ \\
\hline $\mathrm{C}(37)-\mathrm{C}(36)-\mathrm{Cl}(3)$ & $119.7(2)$ \\
\hline $\mathrm{C}(35)-\mathrm{C}(36)-\mathrm{Cl}(3)$ & $119.6(2)$ \\
\hline $\mathrm{C}(38)-\mathrm{C}(37)-\mathrm{C}(36)$ & $120.0(3)$ \\
\hline $\mathrm{C}(37)-\mathrm{C}(38)-\mathrm{C}(33)$ & $120.7(3)$ \\
\hline
\end{tabular}




\begin{tabular}{|c|c|c|c|}
\hline $\mathrm{C}(44)-\mathrm{C}(39)-\mathrm{C}(40)$ & $118.6(3)$ & $\mathrm{C}(56)-\mathrm{C}(55)-\mathrm{C}(54)$ & $119.9(3)$ \\
\hline $\mathrm{C}(44)-\mathrm{C}(39)-\mathrm{C}(17)$ & $119.4(3)$ & $C(55)-C(56)-C(57)$ & $119.1(3)$ \\
\hline$C(40)-C(39)-C(17)$ & $122.0(3)$ & $\mathrm{C}(56)-\mathrm{C}(57)-\mathrm{C}(58)$ & $120.0(3)$ \\
\hline$C(39)-C(40)-C(41)$ & $120.6(3)$ & $\mathrm{C}(57)-\mathrm{C}(58)-\mathrm{C}(53)$ & $123.2(3)$ \\
\hline $\mathrm{C}(42)-\mathrm{C}(41)-\mathrm{C}(40)$ & $119.7(3)$ & $\mathrm{C}(64)-\mathrm{C}(59)-\mathrm{C}(60)$ & $114.6(3)$ \\
\hline $\mathrm{C}(43)-\mathrm{C}(42)-\mathrm{C}(41)$ & $120.2(3)$ & $\mathrm{C}(64)-\mathrm{C}(59)-\mathrm{B}(1)$ & $122.3(3)$ \\
\hline $\mathrm{C}(43)-\mathrm{C}(42)-\mathrm{Cl}(4)$ & $120.4(3)$ & $\mathrm{C}(60)-\mathrm{C}(59)-\mathrm{B}(1)$ & $122.8(3)$ \\
\hline $\mathrm{C}(41)-\mathrm{C}(42)-\mathrm{Cl}(4)$ & $119.3(3)$ & $\mathrm{C}(61)-\mathrm{C}(60)-\mathrm{C}(59)$ & $123.1(3)$ \\
\hline $\mathrm{C}(42)-\mathrm{C}(43)-\mathrm{C}(44)$ & $119.3(3)$ & $\mathrm{C}(62)-\mathrm{C}(61)-\mathrm{C}(60)$ & $120.2(3)$ \\
\hline$C(39)-C(44)-C(43)$ & $121.5(3)$ & $\mathrm{C}(61)-\mathrm{C}(62)-\mathrm{C}(63)$ & $119.0(3)$ \\
\hline $\mathrm{O}(1)-\mathrm{C}(45)-\mathrm{C}(46)$ & 104.1(3) & $\mathrm{C}(62)-\mathrm{C}(63)-\mathrm{C}(64)$ & $120.3(3)$ \\
\hline$C(47)-C(46)-C(45)$ & $102.9(3)$ & $C(63)-C(64)-C(59)$ & $122.8(3)$ \\
\hline$C(46)-C(47)-C(48)$ & $102.6(3)$ & $\mathrm{C}(70)-\mathrm{C}(65)-\mathrm{C}(66)$ & $114.9(3)$ \\
\hline $\mathrm{O}(1)-\mathrm{C}(48)-\mathrm{C}(47)$ & $104.2(3)$ & $\mathrm{C}(70)-\mathrm{C}(65)-\mathrm{B}(1)$ & $123.2(3)$ \\
\hline $\mathrm{O}(2)-\mathrm{C}(49)-\mathrm{C}(50)$ & $104.9(2)$ & $\mathrm{C}(66)-\mathrm{C}(65)-\mathrm{B}(1)$ & $121.9(3)$ \\
\hline$C(49)-C(50)-C(51)$ & $102.8(3)$ & $\mathrm{C}(67)-\mathrm{C}(66)-\mathrm{C}(65)$ & $122.7(3)$ \\
\hline $\mathrm{C}(52)-\mathrm{C}(51)-\mathrm{C}(50)$ & $103.9(2)$ & $\mathrm{C}(66)-\mathrm{C}(67)-\mathrm{C}(68)$ & $119.8(3)$ \\
\hline $\mathrm{O}(2)-\mathrm{C}(52)-\mathrm{C}(51)$ & $106.4(2)$ & $\mathrm{C}(69)-\mathrm{C}(68)-\mathrm{C}(67)$ & $119.8(3)$ \\
\hline $\mathrm{C}(59)-\mathrm{B}(1)-\mathrm{C}(71)$ & $108.5(2)$ & $\mathrm{C}(68)-\mathrm{C}(69)-\mathrm{C}(70)$ & $119.5(3)$ \\
\hline $\mathrm{C}(59)-\mathrm{B}(1)-\mathrm{C}(65)$ & $110.9(2)$ & $\mathrm{C}(69)-\mathrm{C}(70)-\mathrm{C}(65)$ & $123.4(3)$ \\
\hline $\mathrm{C}(71)-\mathrm{B}(1)-\mathrm{C}(65)$ & $107.9(2)$ & $\mathrm{C}(72)-\mathrm{C}(71)-\mathrm{C}(76)$ & $114.6(3)$ \\
\hline $\mathrm{C}(59)-\mathrm{B}(1)-\mathrm{C}(53)$ & $110.2(2)$ & $\mathrm{C}(72)-\mathrm{C}(71)-\mathrm{B}(1)$ & $122.2(3)$ \\
\hline $\mathrm{C}(71)-\mathrm{B}(1)-\mathrm{C}(53)$ & $110.7(2)$ & $\mathrm{C}(76)-\mathrm{C}(71)-\mathrm{B}(1)$ & $123.2(3)$ \\
\hline $\mathrm{C}(65)-\mathrm{B}(1)-\mathrm{C}(53)$ & $108.6(2)$ & $\mathrm{C}(71)-\mathrm{C}(72)-\mathrm{C}(73)$ & $122.9(3)$ \\
\hline $\mathrm{C}(58)-\mathrm{C}(53)-\mathrm{C}(54)$ & $114.0(3)$ & $\mathrm{C}(74)-\mathrm{C}(73)-\mathrm{C}(72)$ & $120.2(3)$ \\
\hline $\mathrm{C}(58)-\mathrm{C}(53)-\mathrm{B}(1)$ & $122.2(3)$ & $C(75)-C(74)-C(73)$ & $118.6(3)$ \\
\hline $\mathrm{C}(54)-\mathrm{C}(53)-\mathrm{B}(1)$ & $123.7(3)$ & $\mathrm{C}(74)-\mathrm{C}(75)-\mathrm{C}(76)$ & $120.4(3)$ \\
\hline $\mathrm{C}(55)-\mathrm{C}(54)-\mathrm{C}(53)$ & $123.7(3)$ & $C(75)-C(76)-C(71)$ & $123.3(3)$ \\
\hline
\end{tabular}

Symmetry transformations used to generate equivalent atoms: 


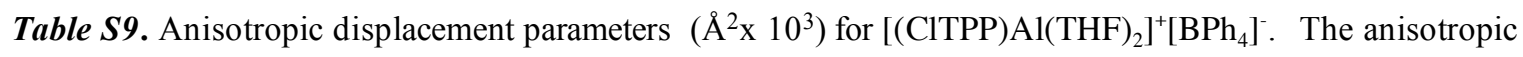
displacement factor exponent takes the form: $-2 \pi^{2}\left[h^{2} a^{* 2} U^{11}+\ldots+2 h k a^{*} b^{*} U^{12}\right]$

\begin{tabular}{|c|c|c|c|c|c|c|}
\hline & $\mathrm{U}^{11}$ & $\mathrm{U}^{22}$ & $\mathrm{U}^{33}$ & $U^{23}$ & $\mathrm{U}^{13}$ & $\mathrm{U}^{12}$ \\
\hline $\mathrm{Cl}(1)$ & $63(1)$ & $39(1)$ & $91(1)$ & $-23(1)$ & $-14(1)$ & $-7(1)$ \\
\hline $\mathrm{Cl}(2)$ & $80(1)$ & $97(1)$ & $71(1)$ & $22(1)$ & $41(1)$ & $5(1)$ \\
\hline $\mathrm{Cl}(3)$ & $90(1)$ & $47(1)$ & $47(1)$ & $-19(1)$ & $0(1)$ & $-22(1)$ \\
\hline $\mathrm{Cl}(4)$ & $49(1)$ & $111(1)$ & $77(1)$ & $-7(1)$ & $21(1)$ & $-5(1)$ \\
\hline $\operatorname{Al}(1)$ & $42(1)$ & $26(1)$ & $19(1)$ & $-2(1)$ & $1(1)$ & $-1(1)$ \\
\hline $\mathrm{O}(1)$ & $47(1)$ & $32(1)$ & $26(1)$ & $-4(1)$ & $-5(1)$ & $4(1)$ \\
\hline $\mathrm{O}(2)$ & $46(1)$ & $28(1)$ & $20(1)$ & $3(1)$ & $3(1)$ & $3(1)$ \\
\hline $\mathrm{N}(1)$ & $47(2)$ & $29(2)$ & $22(1)$ & $-2(1)$ & $0(1)$ & $-3(1)$ \\
\hline $\mathrm{N}(2)$ & $40(2)$ & $28(2)$ & $22(1)$ & $-1(1)$ & $-3(1)$ & $-5(1)$ \\
\hline $\mathrm{N}(3)$ & $44(2)$ & $25(1)$ & $19(1)$ & 1(1) & $1(1)$ & $1(1)$ \\
\hline $\mathrm{N}(4)$ & $39(2)$ & $26(2)$ & $23(1)$ & $-1(1)$ & $0(1)$ & $0(1)$ \\
\hline $\mathrm{C}(1)$ & $51(2)$ & $23(2)$ & $32(2)$ & $-3(2)$ & $-2(2)$ & $-4(2)$ \\
\hline$C(2)$ & $46(2)$ & $28(2)$ & $27(2)$ & $-7(2)$ & $-1(2)$ & $0(2)$ \\
\hline$C(3)$ & $51(2)$ & $22(2)$ & $23(2)$ & $-3(2)$ & $-3(2)$ & $3(2)$ \\
\hline$C(4)$ & $61(2)$ & $30(2)$ & $18(2)$ & $-6(2)$ & $0(2)$ & $1(2)$ \\
\hline$C(5)$ & $51(2)$ & $36(2)$ & $25(2)$ & $2(2)$ & $2(1)$ & $3(2)$ \\
\hline$C(6)$ & $48(2)$ & $25(2)$ & $17(2)$ & $2(2)$ & $3(1)$ & $6(2)$ \\
\hline$C(7)$ & $41(2)$ & $24(2)$ & $23(2)$ & $3(2)$ & $3(1)$ & $5(1)$ \\
\hline$C(8)$ & $38(2)$ & $20(2)$ & $25(2)$ & $1(2)$ & $1(1)$ & $3(1)$ \\
\hline $\mathrm{C}(9)$ & $43(2)$ & $29(2)$ & $30(2)$ & $4(2)$ & $5(1)$ & $-1(2)$ \\
\hline $\mathrm{C}(10)$ & $44(2)$ & $28(2)$ & $30(2)$ & $1(2)$ & $-2(2)$ & $-5(2)$ \\
\hline$C(11)$ & $45(2)$ & $21(2)$ & $23(2)$ & $1(2)$ & $-4(1)$ & $-1(1)$ \\
\hline$C(12)$ & $45(2)$ & $23(2)$ & $20(2)$ & $-2(1)$ & $0(1)$ & $-1(1)$ \\
\hline$C(13)$ & $48(2)$ & $26(2)$ & $20(2)$ & $-1(2)$ & $1(2)$ & $2(2)$ \\
\hline$C(14)$ & $54(2)$ & $34(2)$ & $28(2)$ & $-8(2)$ & $1(2)$ & $-1(2)$ \\
\hline$C(15)$ & $51(2)$ & $34(2)$ & $31(2)$ & $-6(2)$ & $9(2)$ & $-4(2)$ \\
\hline$C(16)$ & $40(2)$ & $33(2)$ & $23(2)$ & $0(2)$ & $0(1)$ & $1(2)$ \\
\hline $\mathrm{C}(17)$ & $41(2)$ & $30(2)$ & $24(2)$ & $-1(2)$ & $1(1)$ & $-1(2)$ \\
\hline $\mathrm{C}(18)$ & $39(2)$ & $34(2)$ & $25(2)$ & $2(2)$ & $-4(2)$ & $-4(2)$ \\
\hline$C(19)$ & $48(2)$ & $41(2)$ & $33(2)$ & $-2(2)$ & $5(2)$ & $-11(2)$ \\
\hline $\mathrm{C}(20)$ & $50(2)$ & $39(2)$ & $38(2)$ & $-10(2)$ & $-1(2)$ & $-9(2)$ \\
\hline
\end{tabular}




\begin{tabular}{|c|c|c|c|c|c|c|}
\hline$C(21)$ & $43(2)$ & $31(2)$ & $26(2)$ & $-3(2)$ & $-3(1)$ & $-2(2)$ \\
\hline$C(22)$ & $45(2)$ & $37(2)$ & $48(2)$ & $-15(2)$ & $-6(2)$ & $6(2)$ \\
\hline$C(23)$ & $40(2)$ & $45(2)$ & $52(2)$ & $-14(2)$ & $-11(2)$ & $5(2)$ \\
\hline$C(24)$ & $48(2)$ & $30(2)$ & $43(2)$ & $-13(2)$ & $-6(2)$ & $-5(2)$ \\
\hline$C(25)$ & $46(2)$ & $29(2)$ & $51(2)$ & $-11(2)$ & $-6(2)$ & $4(2)$ \\
\hline$C(26)$ & $39(2)$ & $40(2)$ & $35(2)$ & $-4(2)$ & $-9(2)$ & $-2(2)$ \\
\hline$C(27)$ & $40(2)$ & $30(2)$ & $23(2)$ & $2(2)$ & $1(1)$ & $3(2)$ \\
\hline$C(28)$ & $55(2)$ & $41(2)$ & $37(2)$ & $4(2)$ & $7(2)$ & $12(2)$ \\
\hline$C(29)$ & $61(3)$ & $55(3)$ & $41(2)$ & $0(2)$ & $16(2)$ & $15(2)$ \\
\hline$C(30)$ & $57(3)$ & $62(3)$ & $33(2)$ & $7(2)$ & $9(2)$ & $3(2)$ \\
\hline $\mathrm{C}(31)$ & $58(2)$ & $43(2)$ & $36(2)$ & $14(2)$ & $8(2)$ & $4(2)$ \\
\hline$C(32)$ & $45(2)$ & $39(2)$ & $29(2)$ & $4(2)$ & $3(2)$ & $5(2)$ \\
\hline$C(33)$ & $41(2)$ & $26(2)$ & $27(2)$ & $-3(2)$ & $5(1)$ & $-3(1)$ \\
\hline$C(34)$ & $62(2)$ & $31(2)$ & $32(2)$ & $0(2)$ & $-5(2)$ & $-6(2)$ \\
\hline$C(35)$ & $78(3)$ & $24(2)$ & $43(2)$ & $-2(2)$ & $-1(2)$ & $-5(2)$ \\
\hline$C(36)$ & $46(2)$ & $33(2)$ & $33(2)$ & $-14(2)$ & $5(2)$ & $-10(2)$ \\
\hline$C(37)$ & $54(2)$ & $36(2)$ & $27(2)$ & $-4(2)$ & $-8(2)$ & $-3(2)$ \\
\hline$C(38)$ & $52(2)$ & $29(2)$ & $31(2)$ & $2(2)$ & $-3(2)$ & $-1(2)$ \\
\hline$C(39)$ & $32(2)$ & $35(2)$ & $30(2)$ & $-2(2)$ & $-1(2)$ & $2(1)$ \\
\hline $\mathrm{C}(40)$ & $33(2)$ & $46(2)$ & $28(2)$ & $-1(2)$ & $0(2)$ & $4(2)$ \\
\hline $\mathrm{C}(41)$ & $44(2)$ & $57(2)$ & $31(2)$ & $4(2)$ & $3(2)$ & $-4(2)$ \\
\hline $\mathrm{C}(42)$ & $39(2)$ & $57(2)$ & $48(2)$ & $-8(2)$ & $9(2)$ & $2(2)$ \\
\hline$C(43)$ & $39(2)$ & $82(3)$ & $56(2)$ & $1(2)$ & $-8(2)$ & $7(2)$ \\
\hline$C(44)$ & $52(3)$ & $66(3)$ & $32(2)$ & $4(2)$ & $-5(2)$ & $3(2)$ \\
\hline$C(45)$ & $73(3)$ & $40(2)$ & $69(3)$ & $-3(2)$ & $-16(2)$ & $12(2)$ \\
\hline$C(46)$ & $79(3)$ & $48(2)$ & $70(3)$ & $5(2)$ & $-16(2)$ & $18(2)$ \\
\hline$C(47)$ & $60(3)$ & $67(3)$ & $54(2)$ & $6(2)$ & $-7(2)$ & $12(2)$ \\
\hline $\mathrm{C}(48)$ & $60(2)$ & $69(3)$ & $41(2)$ & $-6(2)$ & $-13(2)$ & $6(2)$ \\
\hline C(49) & $51(2)$ & $51(2)$ & $39(2)$ & $11(2)$ & $3(2)$ & $14(2)$ \\
\hline$C(50)$ & $55(2)$ & $42(2)$ & $42(2)$ & $11(2)$ & $1(2)$ & $14(2)$ \\
\hline$C(51)$ & $63(2)$ & $33(2)$ & $33(2)$ & $7(2)$ & $-5(2)$ & $1(2)$ \\
\hline$C(52)$ & $63(3)$ & $59(2)$ & $31(2)$ & $18(2)$ & $6(2)$ & $7(2)$ \\
\hline $\mathrm{B}(1)$ & $40(2)$ & $34(2)$ & $28(2)$ & $-3(2)$ & $-4(2)$ & $0(2)$ \\
\hline$C(53)$ & $38(2)$ & $36(2)$ & $20(2)$ & $-1(1)$ & $-3(1)$ & $0(2)$ \\
\hline$C(54)$ & $39(2)$ & $34(2)$ & $36(2)$ & $-6(2)$ & $-4(1)$ & $-1(2)$ \\
\hline$C(55)$ & $51(2)$ & $32(2)$ & $38(2)$ & $-7(2)$ & $-1(2)$ & $-1(2)$ \\
\hline
\end{tabular}




\begin{tabular}{lllllll}
$\mathrm{C}(56)$ & $57(3)$ & $36(2)$ & $35(2)$ & $-3(2)$ & $3(2)$ & $12(2)$ \\
$\mathrm{C}(57)$ & $42(2)$ & $44(2)$ & $36(2)$ & $-1(2)$ & $2(2)$ & $7(2)$ \\
$\mathrm{C}(58)$ & $49(2)$ & $33(2)$ & $28(2)$ & $-1(2)$ & $1(2)$ & $-4(2)$ \\
$\mathrm{C}(59)$ & $36(2)$ & $35(2)$ & $31(2)$ & $-1(2)$ & $2(1)$ & $-2(1)$ \\
$\mathrm{C}(60)$ & $46(2)$ & $43(2)$ & $31(2)$ & $3(2)$ & $3(2)$ & $-4(2)$ \\
$\mathrm{C}(61)$ & $62(3)$ & $57(3)$ & $30(2)$ & $0(2)$ & $-1(2)$ & $-11(2)$ \\
$\mathrm{C}(62)$ & $62(3)$ & $63(3)$ & $35(2)$ & $15(2)$ & $-5(2)$ & $-6(2)$ \\
$\mathrm{C}(63)$ & $57(2)$ & $53(2)$ & $44(2)$ & $11(2)$ & $-2(2)$ & $7(2)$ \\
$\mathrm{C}(64)$ & $61(2)$ & $39(2)$ & $35(2)$ & $-2(2)$ & $-1(2)$ & $6(2)$ \\
$\mathrm{C}(65)$ & $40(2)$ & $25(2)$ & $32(2)$ & $6(2)$ & $-3(1)$ & $4(1)$ \\
$\mathrm{C}(66)$ & $53(2)$ & $35(2)$ & $39(2)$ & $5(2)$ & $-2(2)$ & $-1(2)$ \\
$\mathrm{C}(67)$ & $54(2)$ & $40(2)$ & $57(2)$ & $10(2)$ & $-5(2)$ & $-10(2)$ \\
$\mathrm{C}(68)$ & $66(3)$ & $40(2)$ & $63(3)$ & $-4(2)$ & $-23(2)$ & $-7(2)$ \\
$\mathrm{C}(69)$ & $65(3)$ & $34(2)$ & $39(2)$ & $0(2)$ & $-13(2)$ & $-2(2)$ \\
$\mathrm{C}(70)$ & $47(2)$ & $31(2)$ & $32(2)$ & $4(2)$ & $-7(2)$ & $3(2)$ \\
$\mathrm{C}(71)$ & $42(2)$ & $25(2)$ & $31(2)$ & $-6(2)$ & $-4(1)$ & $-2(1)$ \\
$\mathrm{C}(72)$ & $44(2)$ & $36(2)$ & $40(2)$ & $-6(2)$ & $1(2)$ & $2(2)$ \\
$\mathrm{C}(73)$ & $39(2)$ & $45(2)$ & $62(2)$ & $-13(2)$ & $-6(2)$ & $5(2)$ \\
$\mathrm{C}(74)$ & $43(2)$ & $47(2)$ & $61(2)$ & $-21(2)$ & $16(2)$ & $-5(2)$ \\
$\mathrm{C}(75)$ & $61(3)$ & $35(2)$ & $40(2)$ & $-7(2)$ & $14(2)$ & $-3(2)$ \\
$\mathrm{C}(76)$ & $52(2)$ & $31(2)$ & $32(2)$ & $-7(2)$ & $2(2)$ & $2(2)$ \\
\hline & & & & & & \\
\hline
\end{tabular}


Table S10. Hydrogen coordinates ( $\left.\mathrm{x} 10^{4}\right)$ and isotropic displacement parameters $\left(\AA^{2} \times 10^{3}\right)$ for $\left[(\mathrm{ClTPP}) \mathrm{Al}(\mathrm{THF})_{2}\right]^{+}\left[\mathrm{BPh}_{4}\right]^{-}$.

\begin{tabular}{|c|c|c|c|c|}
\hline & $\mathrm{x}$ & $\mathrm{y}$ & $\mathrm{z}$ & $\mathrm{U}(\mathrm{eq})$ \\
\hline $\mathrm{H}(4 \mathrm{~A})$ & 5713 & 4500 & 3159 & 44 \\
\hline $\mathrm{H}(5 \mathrm{~A})$ & 5116 & 3709 & 3298 & 45 \\
\hline $\mathrm{H}(9 \mathrm{~A})$ & 4277 & 2240 & 1994 & 41 \\
\hline $\mathrm{H}(10 \mathrm{~A})$ & 4490 & 1921 & 1094 & 41 \\
\hline $\mathrm{H}(14 \mathrm{~A})$ & 6149 & 2199 & -178 & 47 \\
\hline $\mathrm{H}(15 \mathrm{~A})$ & 6934 & 2817 & -178 & 46 \\
\hline $\mathrm{H}(19 \mathrm{~A})$ & 7355 & 4672 & 824 & 49 \\
\hline $\mathrm{H}(20 \mathrm{~A})$ & 6979 & 5148 & 1594 & 51 \\
\hline $\mathrm{H}(22 \mathrm{~A})$ & 7148 & 4901 & 2603 & 52 \\
\hline $\mathrm{H}(23 \mathrm{~A})$ & 7349 & 5760 & 2958 & 55 \\
\hline $\mathrm{H}(25 \mathrm{~A})$ & 5810 & 6221 & 2779 & 51 \\
\hline $\mathrm{H}(26 \mathrm{~A})$ & 5611 & 5361 & 2424 & 46 \\
\hline $\mathrm{H}(28 \mathrm{~A})$ & 4209 & 3603 & 2842 & 53 \\
\hline $\mathrm{H}(29 \mathrm{~A})$ & 3524 & 3350 & 3415 & 63 \\
\hline $\mathrm{H}(31 \mathrm{~A})$ & 4113 & 1805 & 3480 & 55 \\
\hline $\mathrm{H}(32 \mathrm{~A})$ & 4795 & 2059 & 2901 & 45 \\
\hline $\mathrm{H}(34 \mathrm{~A})$ & 5418 & 1261 & 779 & 49 \\
\hline $\mathrm{H}(35 \mathrm{~A})$ & 5143 & 567 & 203 & 58 \\
\hline $\mathrm{H}(37 \mathrm{~A})$ & 4692 & 1726 & -845 & 47 \\
\hline $\mathrm{H}(38 \mathrm{~A})$ & 4981 & 2414 & -281 & 45 \\
\hline $\mathrm{H}(40 \mathrm{~A})$ & 6998 & 3845 & -424 & 42 \\
\hline $\mathrm{H}(41 \mathrm{~A})$ & 7751 & 4007 & -943 & 52 \\
\hline $\mathrm{H}(43 \mathrm{~A})$ & 8700 & 3815 & 330 & 71 \\
\hline $\mathrm{H}(44 \mathrm{~A})$ & 7944 & 3654 & 841 & 60 \\
\hline $\mathrm{H}(45 \mathrm{~A})$ & 6743 & 2290 & 1348 & 73 \\
\hline $\mathrm{H}(45 \mathrm{~B})$ & 6224 & 2084 & 1670 & 73 \\
\hline $\mathrm{H}(46 \mathrm{~A})$ & 6702 & 1953 & 2425 & 79 \\
\hline $\mathrm{H}(46 \mathrm{~B})$ & 7167 & 1818 & 2003 & 79 \\
\hline $\mathrm{H}(47 \mathrm{~A})$ & 7500 & 2704 & 2043 & 72 \\
\hline $\mathrm{H}(47 \mathrm{~B})$ & 7359 & 2579 & 2650 & 72 \\
\hline
\end{tabular}




\begin{tabular}{|c|c|c|c|c|}
\hline $\mathrm{H}(48 \mathrm{~A})$ & 6570 & 3051 & 2608 & 68 \\
\hline $\mathrm{H}(48 \mathrm{~B})$ & 6929 & 3417 & 2215 & 68 \\
\hline $\mathrm{H}(49 \mathrm{~A})$ & 4955 & 4332 & 1635 & 56 \\
\hline $\mathrm{H}(49 \mathrm{~B})$ & 4705 & 3728 & 1541 & 56 \\
\hline $\mathrm{H}(50 \mathrm{~A})$ & 4318 & 4537 & 1035 & 56 \\
\hline $\mathrm{H}(50 \mathrm{~B})$ & 4385 & 3954 & 737 & 56 \\
\hline $\mathrm{H}(51 \mathrm{~A})$ & 4886 & 4511 & 199 & 51 \\
\hline $\mathrm{H}(51 \mathrm{~B})$ & 5094 & 4882 & 683 & 51 \\
\hline $\mathrm{H}(52 \mathrm{~A})$ & 5483 & 3853 & 351 & 61 \\
\hline $\mathrm{H}(52 \mathrm{~B})$ & 5810 & 4341 & 634 & 61 \\
\hline $\mathrm{H}(54 \mathrm{~A})$ & 5449 & 2795 & -1366 & 43 \\
\hline $\mathrm{H}(55 \mathrm{~A})$ & 5885 & 1960 & -1328 & 49 \\
\hline $\mathrm{H}(56 \mathrm{~A})$ & 6817 & 1927 & -1306 & 51 \\
\hline $\mathrm{H}(57 \mathrm{~A})$ & 7302 & 2747 & -1342 & 49 \\
\hline $\mathrm{H}(58 \mathrm{~A})$ & 6859 & 3584 & -1400 & 44 \\
\hline $\mathrm{H}(60 \mathrm{~A})$ & 5671 & 3318 & -2350 & 48 \\
\hline $\mathrm{H}(61 \mathrm{~A})$ & 5445 & 3571 & -3199 & 60 \\
\hline $\mathrm{H}(62 \mathrm{~A})$ & 5311 & 4494 & -3410 & 64 \\
\hline $\mathrm{H}(63 \mathrm{~A})$ & 5462 & 5166 & -2771 & 62 \\
\hline $\mathrm{H}(64 \mathrm{~A})$ & 5700 & 4914 & -1923 & 54 \\
\hline $\mathrm{H}(66 \mathrm{~A})$ & 6668 & 4543 & -1685 & 51 \\
\hline $\mathrm{H}(67 \mathrm{~A})$ & 7207 & 5161 & -1235 & 60 \\
\hline $\mathrm{H}(68 \mathrm{~A})$ & 7015 & 5384 & -360 & 68 \\
\hline $\mathrm{H}(69 \mathrm{~A})$ & 6290 & 4974 & 58 & 55 \\
\hline $\mathrm{H}(70 \mathrm{~A})$ & 5765 & 4340 & -390 & 44 \\
\hline $\mathrm{H}(72 \mathrm{~A})$ & 4803 & 4393 & -1603 & 48 \\
\hline $\mathrm{H}(73 \mathrm{~A})$ & 3989 & 4404 & -1167 & 59 \\
\hline $\mathrm{H}(74 \mathrm{~A})$ & 3866 & 3875 & -405 & 60 \\
\hline $\mathrm{H}(75 \mathrm{~A})$ & 4564 & 3322 & -113 & 54 \\
\hline $\mathrm{H}(76 \mathrm{~A})$ & 5381 & 3333 & -534 & 46 \\
\hline
\end{tabular}




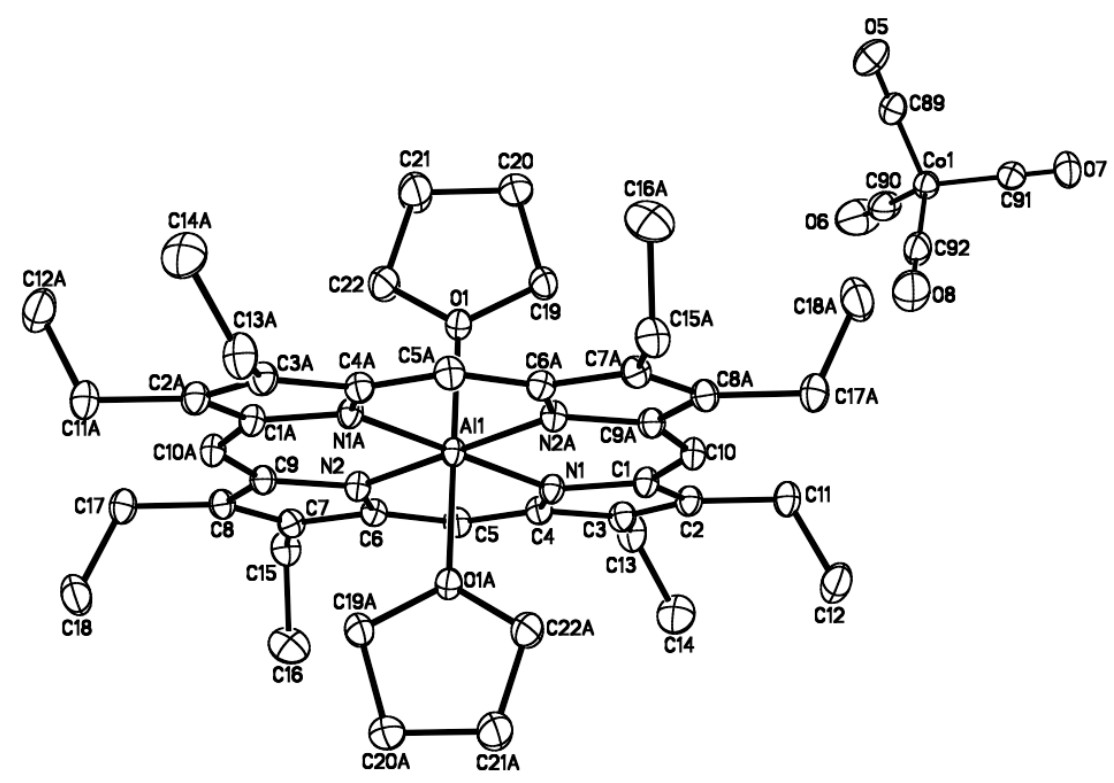

Figure S29. A view of the molecular structure of $\left[(\mathrm{OEP}) \mathrm{Al}(\mathrm{THF})_{2}\right]^{+}\left[\mathrm{Co}(\mathrm{CO})_{4}\right]^{\text {, }}$, (hydrogen atoms omitted for clarity). Displacement ellipsoids are drawn at the $40 \%$ probability level. 
Table S11. Crystal data and structure refinement for $\left[(\mathrm{OEP}) \mathrm{Al}(\mathrm{THF})_{2}\right]^{+}\left[\mathrm{Co}(\mathrm{CO})_{4}\right]^{-}$.

Identification code

Empirical formula

Formula weight

Temperature

Wavelength

Crystal system

Space group

Unit cell dimensions

Volume

Z

Density (calculated)

Absorption coefficient

$\mathrm{F}(000)$

Crystal size

Theta range for data collection

Index ranges

Reflections collected

Independent reflections

Completeness to theta $=31.13^{\circ}$

Absorption correction

Max. and min. transmission

Refinement method

Data / restraints / parameters

Goodness-of-fit on $\mathrm{F}^{2}$

Final $\mathrm{R}$ indices [I $>2 \operatorname{sigma}(\mathrm{I})]$

$\mathrm{R}$ indices (all data)

Largest diff. peak and hole jr2

C48 H60 Al Co N4 O6

874.91

173(2) K

$0.71073 \AA$

Triclinic

P-1

$\mathrm{a}=9.8344(15) \AA$

$\alpha=74.747(8)^{\circ}$

$\mathrm{b}=18.731(3) \AA$

$\beta=86.372(7)^{\circ}$

$\mathrm{c}=25.509(4) \AA$

$\gamma=80.809(7)^{\circ}$

4474.1(12) $\AA^{3}$

4

$1.299 \mathrm{Mg} / \mathrm{m}^{3}$

$0.456 \mathrm{~mm}^{-1}$

1856

$0.50 \times 0.40 \times 0.30 \mathrm{~mm}^{3}$

2.10 to $31.13^{\circ}$.

$-14<=\mathrm{h}<=14,-27<=\mathrm{k}<=27,-36<=1<=36$

115998

28387 [R(int) $=0.0340]$

$98.3 \%$

Semi-empirical from equivalents

0.8753 and 0.8040

Full-matrix least-squares on $\mathrm{F}^{2}$

28387 / 0 / 1260

1.016

$\mathrm{R} 1=0.0561, \mathrm{wR} 2=0.1419$

$\mathrm{R} 1=0.0855, \mathrm{wR} 2=0.1564$

0.924 and -0.547 e. $\AA^{-3}$ 
Table S12. Atomic coordinates ( x 104) and equivalent isotropic displacement parameters $\left(\AA^{2} \times 10^{3}\right)$ for $\left[(\mathrm{OEP}) \mathrm{Al}(\mathrm{THF})_{2}\right]^{+}\left[\mathrm{Co}(\mathrm{CO})_{4}\right]^{-}$. U(eq) is defined as one third of the trace of the orthogonalized $\mathrm{U}^{\mathrm{ij}}$ tensor.

\begin{tabular}{|c|c|c|c|c|}
\hline- & $\mathrm{x}$ & $\mathrm{y}$ & $\mathrm{z}$ & $\mathrm{U}(\mathrm{eq})$ \\
\hline $\mathrm{Co}(1)$ & 1343(1) & $70(1)$ & $2455(1)$ & $38(1)$ \\
\hline $\mathrm{Co}(2)$ & $3605(1)$ & $4858(1)$ & 7494(1) & $42(1)$ \\
\hline $\operatorname{Al}(1)$ & 5000 & 5000 & 10000 & $18(1)$ \\
\hline $\mathrm{Al}(2)$ & 0 & 0 & 0 & $18(1)$ \\
\hline $\operatorname{Al}(3)$ & $7660(1)$ & $2466(1)$ & 4951(1) & $34(1)$ \\
\hline $\mathrm{O}(1)$ & $3280(1)$ & $5277(1)$ & $9558(1)$ & $22(1)$ \\
\hline $\mathrm{O}(2)$ & $-1786(1)$ & $414(1)$ & $-389(1)$ & $24(1)$ \\
\hline $\mathrm{O}(3)$ & $6485(1)$ & 3391(1) & $4550(1)$ & $40(1)$ \\
\hline $\mathrm{O}(4)$ & $8831(2)$ & $1545(1)$ & $5357(1)$ & $40(1)$ \\
\hline $\mathrm{O}(5)$ & $-1267(2)$ & $964(1)$ & $2629(1)$ & $63(1)$ \\
\hline $\mathrm{O}(6)$ & $721(2)$ & $-1401(1)$ & $2464(1)$ & $96(1)$ \\
\hline $\mathrm{O}(7)$ & $2519(2)$ & $747(1)$ & $1402(1)$ & $55(1)$ \\
\hline $\mathrm{O}(8)$ & $3323(2)$ & $-1(2)$ & $3279(1)$ & $85(1)$ \\
\hline $\mathrm{O}(9)$ & $1400(2)$ & $5118(2)$ & $6723(1)$ & $84(1)$ \\
\hline $\mathrm{O}(10)$ & $6125(2)$ & $4208(1)$ & 7033(1) & $68(1)$ \\
\hline $\mathrm{O}(11)$ & $4166(3)$ & $6251(1)$ & $7672(1)$ & $77(1)$ \\
\hline $\mathrm{O}(12)$ & $2907(2)$ & $3860(1)$ & $8527(1)$ & $67(1)$ \\
\hline $\mathrm{N}(1)$ & 5983(1) & $4629(1)$ & 9393(1) & $20(1)$ \\
\hline $\mathrm{N}(2)$ & $4473(1)$ & 3993(1) & 10331(1) & $20(1)$ \\
\hline $\mathrm{N}(3)$ & $1032(1)$ & $447(1)$ & $-671(1)$ & $21(1)$ \\
\hline $\mathrm{N}(4)$ & $-61(1)$ & $936(1)$ & $237(1)$ & $22(1)$ \\
\hline $\mathrm{N}(5)$ & $9010(2)$ & $2572(1)$ & $4331(1)$ & $35(1)$ \\
\hline $\mathrm{N}(6)$ & $6684(2)$ & $1840(1)$ & $4622(1)$ & $33(1)$ \\
\hline $\mathrm{N}(7)$ & $6306(2)$ & $2362(1)$ & $5568(1)$ & $40(1)$ \\
\hline $\mathrm{N}(8)$ & $8639(2)$ & 3091(1) & $5279(1)$ & $36(1)$ \\
\hline$C(1)$ & $6638(2)$ & $5033(1)$ & $8944(1)$ & $22(1)$ \\
\hline$C(2)$ & $7031(2)$ & $4599(1)$ & $8553(1)$ & $23(1)$ \\
\hline$C(3)$ & $6585(2)$ & $3926(1)$ & $8760(1)$ & $24(1)$ \\
\hline$C(4)$ & $5951(2)$ & $3948(1)$ & $9282(1)$ & $21(1)$ \\
\hline$C(5)$ & $5370(2)$ & $3370(1)$ & $9623(1)$ & $22(1)$ \\
\hline$C(6)$ & $4695(2)$ & $3383(1)$ & 10111(1) & $20(1)$ \\
\hline
\end{tabular}




\begin{tabular}{|c|c|c|c|c|}
\hline$C(7)$ & $4067(2)$ & $2771(1)$ & $10450(1)$ & $22(1)$ \\
\hline $\mathrm{C}(8)$ & $3456(2)$ & $3011(1)$ & $10880(1)$ & $22(1)$ \\
\hline $\mathrm{C}(9)$ & $3702(2)$ & $3773(1)$ & 10799(1) & $21(1)$ \\
\hline $\mathrm{C}(10)$ & $6804(2)$ & $5774(1)$ & $8863(1)$ & $23(1)$ \\
\hline $\mathrm{C}(11)$ & $7750(2)$ & $4870(1)$ & $8022(1)$ & $28(1)$ \\
\hline $\mathrm{C}(12)$ & $9281(2)$ & 4861(1) & $8081(1)$ & $41(1)$ \\
\hline $\mathrm{C}(13)$ & $6665(2)$ & $3293(1)$ & $8491(1)$ & $32(1)$ \\
\hline$C(14)$ & $8094(2)$ & $2918(1)$ & $8406(1)$ & $44(1)$ \\
\hline$C(15)$ & $4098(2)$ & $2022(1)$ & $10330(1)$ & $28(1)$ \\
\hline$C(16)$ & $5401(2)$ & $1484(1)$ & 10521(1) & $43(1)$ \\
\hline $\mathrm{C}(17)$ & $2682(2)$ & $2590(1)$ & $11352(1)$ & $28(1)$ \\
\hline $\mathrm{C}(18)$ & $3568(2)$ & 2233(1) & $11848(1)$ & $43(1)$ \\
\hline C(19) & $3293(2)$ & $5556(1)$ & $8965(1)$ & $30(1)$ \\
\hline $\mathrm{C}(20)$ & $1794(2)$ & $5731(1)$ & $8809(1)$ & $37(1)$ \\
\hline $\mathrm{C}(21)$ & $1025(2)$ & $5324(2)$ & $9285(1)$ & $59(1)$ \\
\hline $\mathrm{C}(22)$ & $2045(2)$ & 4935(1) & $9709(1)$ & $39(1)$ \\
\hline $\mathrm{C}(23)$ & $1442(2)$ & $131(1)$ & $-1097(1)$ & $22(1)$ \\
\hline $\mathrm{C}(24)$ & $2139(2)$ & $629(1)$ & $-1515(1)$ & $26(1)$ \\
\hline$C(25)$ & $2178(2)$ & $1249(1)$ & $-1337(1)$ & $27(1)$ \\
\hline$C(26)$ & $1459(2)$ & 1139(1) & $-818(1)$ & $23(1)$ \\
\hline$C(27)$ & $1178(2)$ & $1669(1)$ & $-522(1)$ & $25(1)$ \\
\hline$C(28)$ & $452(2)$ & $1580(1)$ & $-33(1)$ & $22(1)$ \\
\hline C(29) & $196(2)$ & 2132(1) & $279(1)$ & $24(1)$ \\
\hline$C(30)$ & $-444(2)$ & 1812(1) & $749(1)$ & $25(1)$ \\
\hline $\mathrm{C}(31)$ & $-601(2)$ & $1069(1)$ & $722(1)$ & $22(1)$ \\
\hline$C(32)$ & $-1210(2)$ & $567(1)$ & $1123(1)$ & $25(1)$ \\
\hline C(33) & $2705(2)$ & $479(1)$ & $-2042(1)$ & $33(1)$ \\
\hline$C(34)$ & $4158(3)$ & $48(2)$ & $-2000(1)$ & $53(1)$ \\
\hline$C(35)$ & $2799(2)$ & 1934(1) & $-1626(1)$ & $34(1)$ \\
\hline$C(36)$ & $1799(3)$ & $2518(1)$ & $-1998(1)$ & $48(1)$ \\
\hline$C(37)$ & $519(2)$ & $2925(1)$ & $80(1)$ & $29(1)$ \\
\hline$C(38)$ & $-588(3)$ & $3422(1)$ & $-282(1)$ & $60(1)$ \\
\hline$C(39)$ & $-900(2)$ & 2133(1) & $1225(1)$ & $32(1)$ \\
\hline $\mathrm{C}(40)$ & $-2448(2)$ & $2350(1)$ & $1277(1)$ & $51(1)$ \\
\hline $\mathrm{C}(41)$ & $-2060(2)$ & 1122(1) & $-791(1)$ & $51(1)$ \\
\hline $\mathrm{C}(42)$ & $-3268(2)$ & 1093(1) & $-1090(1)$ & $52(1)$ \\
\hline
\end{tabular}




\begin{tabular}{|c|c|c|c|c|}
\hline$C(43)$ & $-4050(2)$ & $540(1)$ & $-706(1)$ & $37(1)$ \\
\hline$C(44)$ & $-2964(2)$ & $17(1)$ & $-339(1)$ & $33(1)$ \\
\hline$C(45)$ & 9879(4) & $3141(3)$ & $4158(2)$ & $23(1)$ \\
\hline$C(46)$ & 10531(4) & $3119(3)$ & $3637(2)$ & $24(1)$ \\
\hline$C\left(45^{\prime}\right)$ & 10259(4) & $2845(2)$ & $4312(2)$ & $23(1)$ \\
\hline$C\left(46^{\prime}\right)$ & $10989(4)$ & $2797(2)$ & $3813(2)$ & $27(1)$ \\
\hline$C(47)$ & $10128(2)$ & $2515(1)$ & $3511(1)$ & $45(1)$ \\
\hline$C(48)$ & $9029(2)$ & $2286(1)$ & $3885(1)$ & $27(1)$ \\
\hline$C(49)$ & $8118(2)$ & $1835(1)$ & $3806(1)$ & $25(1)$ \\
\hline$C(50)$ & $7019(2)$ & $1632(1)$ & $4146(1)$ & $24(1)$ \\
\hline$C(51)$ & $6068(2)$ & $1175(1)$ & $4045(1)$ & $27(1)$ \\
\hline$C(52)$ & $5150(2)$ & $1098(1)$ & $4466(1)$ & $40(1)$ \\
\hline $\mathrm{C}(53)$ & $5542(2)$ & $1510(2)$ & $4824(1)$ & $53(1)$ \\
\hline$C(54)$ & $5058(4)$ & $1417(2)$ & $5360(2)$ & $32(1)$ \\
\hline $\mathrm{C}(55)$ & $5386(4)$ & $1812(2)$ & $5708(1)$ & $27(1)$ \\
\hline$C(56)$ & $4698(4)$ & $1837(2)$ & $6219(1)$ & $29(1)$ \\
\hline$C\left(54^{\prime}\right)$ & $4636(5)$ & $1769(3)$ & $5235(2)$ & $23(1)$ \\
\hline$C\left(55^{\prime}\right)$ & $5034(5)$ & 2137(3) & $5592(2)$ & $21(1)$ \\
\hline$C\left(56^{\prime}\right)$ & $4325(5)$ & 2196(3) & $6098(2)$ & $27(1)$ \\
\hline $\mathrm{C}(57)$ & $5174(2)$ & $2426(2)$ & $6385(1)$ & $48(1)$ \\
\hline $\mathrm{C}(58)$ & $6262(2)$ & $2666(1)$ & $6006(1)$ & $32(1)$ \\
\hline $\mathrm{C}(59)$ & $7156(2)$ & $3130(1)$ & $6080(1)$ & $30(1)$ \\
\hline$C(60)$ & $8270(2)$ & $3326(1)$ & $5743(1)$ & $27(1)$ \\
\hline $\mathrm{C}(61)$ & $9199(2)$ & $3805(1)$ & $5834(1)$ & $29(1)$ \\
\hline$C(62)$ & 10141(2) & $3860(1)$ & $5420(1)$ & $41(1)$ \\
\hline$C(63)$ & $9633(5)$ & $3561(3)$ & $5003(2)$ & $23(1)$ \\
\hline$C(64)$ & 10217(4) & $3550(3)$ & $4498(2)$ & $25(1)$ \\
\hline$C(65)$ & $11597(5)$ & $3580(3)$ & $3354(2)$ & $32(1)$ \\
\hline$C(66)$ & $13028(5)$ & $3153(3)$ & $3354(2)$ & $48(1)$ \\
\hline$C\left(63^{\prime}\right)$ & 9894(4) & $3310(2)$ & $5133(2)$ & $22(1)$ \\
\hline$C\left(64^{\prime}\right)$ & $10627(4)$ & $3214(2)$ & $4673(2)$ & $27(1)$ \\
\hline$C\left(65^{\prime}\right)$ & 12295(4) & $3111(2)$ & $3617(2)$ & $37(1)$ \\
\hline$C\left(66^{\prime}\right)$ & 12031(7) & 3904(4) & $3280(3)$ & $79(2)$ \\
\hline$C(67)$ & $10426(3)$ & $2326(2)$ & $2975(1)$ & $51(1)$ \\
\hline$C(68)$ & $9510(4)$ & $2814(2)$ & $2530(1)$ & $81(1)$ \\
\hline $\mathrm{C}(69)$ & $6100(2)$ & $873(1)$ & $3557(1)$ & $35(1)$ \\
\hline
\end{tabular}




\begin{tabular}{|c|c|c|c|c|}
\hline$C(70)$ & $5514(3)$ & $1451(2)$ & $3055(1)$ & $54(1)$ \\
\hline $\mathrm{C}(71)$ & 3918(3) & $692(2)$ & $4545(1)$ & $58(1)$ \\
\hline$C(72)$ & 2656(3) & $1176(2)$ & $4283(2)$ & $81(1)$ \\
\hline$C(73)$ & $3579(5)$ & 1404(3) & $6482(2)$ & $38(1)$ \\
\hline$C(74)$ & $2150(4)$ & $1849(3)$ & $6431(2)$ & $61(1)$ \\
\hline$C\left(73^{\prime}\right)$ & $3033(5)$ & $1855(3)$ & $6310(2)$ & $40(1)$ \\
\hline$C\left(74^{\prime}\right)$ & $3343(7)$ & $1068(4)$ & $6532(3)$ & $58(2)$ \\
\hline$C(75)$ & $4842(2)$ & $2643(2)$ & $6910(1)$ & $52(1)$ \\
\hline$C(76)$ & $5849(3)$ & $2244(2)$ & $7348(1)$ & $61(1)$ \\
\hline$C(77)$ & $9114(2)$ & $4152(1)$ & 6303(1) & $37(1)$ \\
\hline$C(78)$ & $9690(3)$ & $3616(2)$ & $6824(1)$ & $60(1)$ \\
\hline$C(79)$ & $11346(2)$ & $4284(2)$ & $5327(1)$ & $52(1)$ \\
\hline $\mathrm{C}(80)$ & 12643(3) & $3810(2)$ & $5580(2)$ & $85(1)$ \\
\hline $\mathrm{C}(81)$ & $6120(5)$ & $4172(2)$ & $4679(2)$ & $47(1)$ \\
\hline $\mathrm{C}(82)$ & $5134(6)$ & 4694(3) & $4268(2)$ & $48(1)$ \\
\hline$C(83)$ & $4580(8)$ & $4173(4)$ & 4001(3) & $51(2)$ \\
\hline $\mathrm{C}(84)$ & $5807(4)$ & $3592(2)$ & $3983(2)$ & $44(1)$ \\
\hline $\mathrm{C}\left(81^{\prime}\right)$ & $7023(5)$ & $4000(3)$ & $4302(3)$ & $60(2)$ \\
\hline $\mathrm{C}\left(82^{\prime}\right)$ & $5799(7)$ & $4555(3)$ & $4215(3)$ & $49(2)$ \\
\hline $\mathrm{C}\left(83^{\prime}\right)$ & $4635(10)$ & $4166(6)$ & $4222(6)$ & $91(5)$ \\
\hline $\mathrm{C}\left(84^{\prime}\right)$ & $5129(5)$ & $3351(3)$ & 4471(3) & $55(2)$ \\
\hline $\mathrm{C}(85)$ & 10183(5) & 1592(3) & $5428(3)$ & $56(2)$ \\
\hline$C(86)$ & $10735(5)$ & $741(4)$ & $5601(3)$ & $62(2)$ \\
\hline $\mathrm{C}(87)$ & $9515(6)$ & $426(3)$ & $5877(3)$ & $56(2)$ \\
\hline $\mathrm{C}(88)$ & $8258(5)$ & $952(3)$ & $5639(3)$ & $61(2)$ \\
\hline$C\left(85^{\prime}\right)$ & $9164(5)$ & $1260(3)$ & $5970(2)$ & $54(1)$ \\
\hline$C\left(86^{\prime}\right)$ & 10342(9) & $638(5)$ & $6028(3)$ & 103(3) \\
\hline $\mathrm{C}\left(87^{\prime}\right)$ & $10221(12)$ & 299(4) & $5620(4)$ & $114(4)$ \\
\hline $\mathrm{C}\left(88^{\prime}\right)$ & $9359(6)$ & $813(3)$ & $5182(2)$ & $58(1)$ \\
\hline $\mathrm{C}(89)$ & $-226(2)$ & $610(1)$ & $2566(1)$ & $42(1)$ \\
\hline $\mathrm{C}(90)$ & $989(3)$ & $-827(2)$ & $2467(1)$ & $57(1)$ \\
\hline $\mathrm{C}(91)$ & $2050(2)$ & $476(1)$ & $1818(1)$ & $40(1)$ \\
\hline $\mathrm{C}(92)$ & $2549(3)$ & $23(2)$ & 2952(1) & $54(1)$ \\
\hline $\mathrm{C}(93)$ & $2257(3)$ & $5012(2)$ & $7034(1)$ & $55(1)$ \\
\hline $\mathrm{C}(94)$ & $5117(3)$ & $4461(2)$ & $7217(1)$ & $50(1)$ \\
\hline$C(95)$ & 3922(3) & $5707(2)$ & $7599(1)$ & $51(1)$ \\
\hline
\end{tabular}


Table S13. Bond lengths $[\AA]$ and angles $\left[{ }^{\circ}\right]$ for $\left[(\mathrm{OEP}) \mathrm{Al}(\mathrm{THF})_{2}\right]^{+}\left[\mathrm{Co}(\mathrm{CO})_{4}\right]^{-}$.

\begin{tabular}{|c|c|c|c|}
\hline $\mathrm{Co}(1)-\mathrm{C}(91)$ & $1.755(2)$ & $\mathrm{O}(4)-\mathrm{C}(88)$ & $1.340(5)$ \\
\hline $\mathrm{Co}(1)-\mathrm{C}(89)$ & $1.756(2)$ & $\mathrm{O}(4)-\mathrm{C}(85)$ & $1.372(5)$ \\
\hline $\mathrm{Co}(1)-\mathrm{C}(90)$ & $1.761(3)$ & $\mathrm{O}(4)-\mathrm{C}\left(88^{\prime}\right)$ & $1.552(5)$ \\
\hline $\mathrm{Co}(1)-\mathrm{C}(92)$ & $1.766(3)$ & $\mathrm{O}(4)-\mathrm{C}\left(85^{\prime}\right)$ & $1.553(4)$ \\
\hline $\mathrm{Co}(2)-\mathrm{C}(94)$ & $1.748(2)$ & $\mathrm{O}(5)-\mathrm{C}(89)$ & $1.154(3)$ \\
\hline $\mathrm{Co}(2)-\mathrm{C}(95)$ & $1.758(3)$ & $\mathrm{O}(6)-\mathrm{C}(90)$ & $1.149(3)$ \\
\hline $\mathrm{Co}(2)-\mathrm{C}(96)$ & $1.761(3)$ & $\mathrm{O}(7)-\mathrm{C}(91)$ & $1.151(3)$ \\
\hline $\mathrm{Co}(2)-\mathrm{C}(93)$ & $1.764(3)$ & $\mathrm{O}(8)-\mathrm{C}(92)$ & $1.153(3)$ \\
\hline $\mathrm{Al}(1)-\mathrm{N}(2) \# 1$ & $1.9905(13)$ & $\mathrm{O}(9)-\mathrm{C}(93)$ & $1.152(3)$ \\
\hline $\mathrm{Al}(1)-\mathrm{N}(2)$ & $1.9905(13)$ & $\mathrm{O}(10)-\mathrm{C}(94)$ & $1.155(3)$ \\
\hline $\mathrm{Al}(1)-\mathrm{N}(1)$ & $1.9951(13)$ & $\mathrm{O}(11)-\mathrm{C}(95)$ & $1.146(3)$ \\
\hline $\mathrm{Al}(1)-\mathrm{N}(1) \# 1$ & $1.9951(13)$ & $\mathrm{O}(12)-\mathrm{C}(96)$ & $1.154(3)$ \\
\hline $\mathrm{Al}(1)-\mathrm{O}(1)$ & $2.0130(12)$ & $\mathrm{N}(1)-\mathrm{C}(1)$ & $1.380(2)$ \\
\hline $\mathrm{Al}(1)-\mathrm{O}(1) \# 1$ & $2.0130(12)$ & $\mathrm{N}(1)-\mathrm{C}(4)$ & $1.383(2)$ \\
\hline $\operatorname{Al}(2)-N(3)$ & $1.9887(13)$ & $\mathrm{N}(2)-\mathrm{C}(9)$ & $1.377(2)$ \\
\hline $\mathrm{Al}(2)-\mathrm{N}(3) \# 2$ & $1.9887(13)$ & $\mathrm{N}(2)-\mathrm{C}(6)$ & $1.382(2)$ \\
\hline $\mathrm{Al}(2)-\mathrm{N}(4) \# 2$ & $1.9898(13)$ & $\mathrm{N}(3)-\mathrm{C}(26)$ & $1.377(2)$ \\
\hline $\operatorname{Al}(2)-\mathrm{N}(4)$ & $1.9898(13)$ & $\mathrm{N}(3)-\mathrm{C}(23)$ & $1.381(2)$ \\
\hline $\mathrm{Al}(2)-\mathrm{O}(2) \# 2$ & $2.0125(12)$ & $\mathrm{N}(4)-\mathrm{C}(28)$ & $1.377(2)$ \\
\hline $\mathrm{Al}(2)-\mathrm{O}(2)$ & $2.0126(12)$ & $\mathrm{N}(4)-\mathrm{C}(31)$ & $1.384(2)$ \\
\hline $\operatorname{Al}(3)-\mathrm{N}(7)$ & $1.9854(16)$ & $\mathrm{N}(5)-\mathrm{C}(48)$ & $1.377(2)$ \\
\hline $\mathrm{Al}(3)-\mathrm{O}(4)$ & $1.9890(18)$ & $\mathrm{N}(5)-\mathrm{C}\left(45^{\prime}\right)$ & $1.397(4)$ \\
\hline $\mathrm{Al}(3)-\mathrm{O}(3)$ & $1.9890(18)$ & $\mathrm{N}(5)-\mathrm{C}(45)$ & $1.434(4)$ \\
\hline $\operatorname{Al}(3)-\mathrm{N}(5)$ & $1.9897(15)$ & $\mathrm{N}(6)-\mathrm{C}(50)$ & $1.375(2)$ \\
\hline $\mathrm{Al}(3)-\mathrm{N}(8)$ & $1.9937(16)$ & $\mathrm{N}(6)-\mathrm{C}(53)$ & $1.375(2)$ \\
\hline $\operatorname{Al}(3)-\mathrm{N}(6)$ & $1.9950(16)$ & $\mathrm{N}(7)-\mathrm{C}\left(55^{\prime}\right)$ & $1.376(5)$ \\
\hline $\mathrm{O}(1)-\mathrm{C}(22)$ & $1.447(2)$ & $\mathrm{N}(7)-\mathrm{C}(58)$ & $1.378(2)$ \\
\hline $\mathrm{O}(1)-\mathrm{C}(19)$ & $1.465(2)$ & $\mathrm{N}(7)-\mathrm{C}(55)$ & $1.439(4)$ \\
\hline $\mathrm{O}(2)-\mathrm{C}(41)$ & $1.446(2)$ & $\mathrm{N}(8)-\mathrm{C}\left(63^{\prime}\right)$ & $1.361(4)$ \\
\hline $\mathrm{O}(2)-\mathrm{C}(44)$ & $1.456(2)$ & $\mathrm{N}(8)-\mathrm{C}(60)$ & $1.376(2)$ \\
\hline $\mathrm{O}(3)-\mathrm{C}\left(81^{\prime}\right)$ & $1.324(5)$ & N(8)-C(63) & $1.445(5)$ \\
\hline $\mathrm{O}(3)-\mathrm{C}\left(84^{\prime}\right)$ & $1.378(5)$ & $C(1)-C(10)$ & $1.382(2)$ \\
\hline $\mathrm{O}(3)-\mathrm{C}(84)$ & $1.561(4)$ & $C(1)-C(2)$ & $1.444(2)$ \\
\hline $\mathrm{O}(3)-\mathrm{C}(81)$ & $1.564(4)$ & $\mathrm{C}(2)-\mathrm{C}(3)$ & $1.363(2)$ \\
\hline
\end{tabular}




\begin{tabular}{|c|c|c|c|}
\hline$C(2)-C(11)$ & $1.491(2)$ & $\mathrm{C}(37)-\mathrm{C}(38)$ & $1.502(3)$ \\
\hline$C(3)-C(4)$ & $1.441(2)$ & $C(39)-C(40)$ & $1.519(3)$ \\
\hline$C(3)-C(13)$ & $1.507(2)$ & $C(41)-C(42)$ & $1.467(3)$ \\
\hline$C(4)-C(5)$ & $1.377(2)$ & $C(42)-C(43)$ & $1.500(3)$ \\
\hline$C(5)-C(6)$ & $1.379(2)$ & $\mathrm{C}(43)-\mathrm{C}(44)$ & $1.512(3)$ \\
\hline$C(6)-C(7)$ & $1.441(2)$ & $\mathrm{C}(45)-\mathrm{C}(64)$ & $1.382(6)$ \\
\hline$C(7)-C(8)$ & $1.362(2)$ & $\mathrm{C}(45)-\mathrm{C}(46)$ & $1.446(5)$ \\
\hline$C(7)-C(15)$ & $1.508(2)$ & $\mathrm{C}(46)-\mathrm{C}(47)$ & $1.375(4)$ \\
\hline $\mathrm{C}(8)-\mathrm{C}(9)$ & $1.444(2)$ & $\mathrm{C}(46)-\mathrm{C}(65)$ & $1.495(6)$ \\
\hline$C(8)-C(17)$ & $1.493(2)$ & $\mathrm{C}\left(45^{\prime}\right)-\mathrm{C}\left(64^{\prime}\right)$ & $1.382(5)$ \\
\hline$C(9)-C(10) \# 1$ & $1.381(2)$ & $C\left(45^{\prime}\right)-C\left(46^{\prime}\right)$ & $1.437(5)$ \\
\hline$C(10)-C(9) \# 1$ & $1.381(2)$ & $\mathrm{C}\left(46^{\prime}\right)-\mathrm{C}(47)$ & $1.425(4)$ \\
\hline$C(11)-C(12)$ & $1.520(3)$ & $\mathrm{C}\left(46^{\prime}\right)-\mathrm{C}\left(65^{\prime}\right)$ & $1.500(5)$ \\
\hline$C(13)-C(14)$ & $1.498(3)$ & $\mathrm{C}(47)-\mathrm{C}(48)$ & $1.438(2)$ \\
\hline$C(15)-C(16)$ & $1.518(3)$ & $\mathrm{C}(47)-\mathrm{C}(67)$ & $1.501(3)$ \\
\hline$C(17)-C(18)$ & $1.522(3)$ & $\mathrm{C}(48)-\mathrm{C}(49)$ & $1.382(2)$ \\
\hline$C(19)-C(20)$ & $1.513(3)$ & $\mathrm{C}(49)-\mathrm{C}(50)$ & $1.380(2)$ \\
\hline$C(20)-C(21)$ & $1.487(3)$ & $\mathrm{C}(50)-\mathrm{C}(51)$ & $1.442(2)$ \\
\hline$C(21)-C(22)$ & $1.481(3)$ & $\mathrm{C}(51)-\mathrm{C}(52)$ & $1.351(3)$ \\
\hline $\mathrm{C}(23)-\mathrm{C}(32) \# 2$ & $1.381(2)$ & $\mathrm{C}(51)-\mathrm{C}(69)$ & $1.494(3)$ \\
\hline$C(23)-C(24)$ & $1.437(2)$ & $\mathrm{C}(52)-\mathrm{C}(53)$ & $1.445(3)$ \\
\hline$C(24)-C(25)$ & $1.361(3)$ & $\mathrm{C}(52)-\mathrm{C}(71)$ & $1.506(3)$ \\
\hline$C(24)-C(33)$ & $1.500(2)$ & $\mathrm{C}(53)-\mathrm{C}(54)$ & $1.396(4)$ \\
\hline$C(25)-C(26)$ & $1.444(2)$ & $\mathrm{C}(53)-\mathrm{C}\left(54^{\prime}\right)$ & $1.464(5)$ \\
\hline$C(25)-C(35)$ & $1.503(2)$ & $\mathrm{C}(54)-\mathrm{C}(55)$ & $1.379(5)$ \\
\hline$C(26)-C(27)$ & $1.381(2)$ & $\mathrm{C}(55)-\mathrm{C}(56)$ & $1.438(5)$ \\
\hline$C(27)-C(28)$ & $1.381(2)$ & $\mathrm{C}(56)-\mathrm{C}(57)$ & $1.432(4)$ \\
\hline$C(28)-C(29)$ & $1.446(2)$ & $\mathrm{C}(56)-\mathrm{C}(73)$ & $1.491(5)$ \\
\hline$C(29)-C(30)$ & $1.357(2)$ & $\mathrm{C}\left(54^{\prime}\right)-\mathrm{C}\left(55^{\prime}\right)$ & $1.384(6)$ \\
\hline$C(29)-C(37)$ & $1.516(2)$ & $\mathrm{C}\left(55^{\prime}\right)-\mathrm{C}\left(56^{\prime}\right)$ & $1.449(6)$ \\
\hline$C(30)-C(31)$ & $1.446(2)$ & $\mathrm{C}\left(56^{\prime}\right)-\mathrm{C}(57)$ & $1.333(5)$ \\
\hline $\mathrm{C}(30)-\mathrm{C}(39)$ & $1.504(2)$ & $\mathrm{C}\left(56^{\prime}\right)-\mathrm{C}\left(73^{\prime}\right)$ & $1.518(6)$ \\
\hline$C(31)-C(32)$ & $1.373(2)$ & $\mathrm{C}(57)-\mathrm{C}(58)$ & $1.441(3)$ \\
\hline $\mathrm{C}(32)-\mathrm{C}(23) \# 2$ & $1.381(2)$ & $\mathrm{C}(57)-\mathrm{C}(75)$ & $1.503(3)$ \\
\hline $\mathrm{C}(33)-\mathrm{C}(34)$ & $1.520(3)$ & $\mathrm{C}(58)-\mathrm{C}(59)$ & $1.383(3)$ \\
\hline$C(35)-C(36)$ & $1.513(3)$ & $\mathrm{C}(59)-\mathrm{C}(60)$ & $1.384(2)$ \\
\hline
\end{tabular}




\begin{tabular}{|c|c|c|c|}
\hline $\mathrm{C}(60)-\mathrm{C}(61)$ & $1.446(2)$ & $\mathrm{C}(90)-\mathrm{Co}(1)-\mathrm{C}(92)$ & $111.24(13)$ \\
\hline $\mathrm{C}(61)-\mathrm{C}(62)$ & $1.354(3)$ & $\mathrm{C}(94)-\mathrm{Co}(2)-\mathrm{C}(95)$ & $107.14(12)$ \\
\hline$C(61)-C(77)$ & $1.496(3)$ & $\mathrm{C}(94)-\mathrm{Co}(2)-\mathrm{C}(96)$ & $110.19(12)$ \\
\hline $\mathrm{C}(62)-\mathrm{C}\left(63^{\prime}\right)$ & $1.469(5)$ & $\mathrm{C}(95)-\mathrm{Co}(2)-\mathrm{C}(96)$ & $110.01(11)$ \\
\hline $\mathrm{C}(62)-\mathrm{C}(63)$ & $1.472(6)$ & $\mathrm{C}(94)-\mathrm{Co}(2)-\mathrm{C}(93)$ & $109.06(12)$ \\
\hline $\mathrm{C}(62)-\mathrm{C}(79)$ & $1.501(3)$ & $\mathrm{C}(95)-\mathrm{Co}(2)-\mathrm{C}(93)$ & $110.91(13)$ \\
\hline $\mathrm{C}(63)-\mathrm{C}(64)$ & $1.383(7)$ & $\mathrm{C}(96)-\mathrm{Co}(2)-\mathrm{C}(93)$ & $109.50(12)$ \\
\hline $\mathrm{C}(65)-\mathrm{C}(66)$ & $1.504(7)$ & $\mathrm{N}(2) \# 1-\mathrm{Al}(1)-\mathrm{N}(2)$ & $180.00(7)$ \\
\hline $\mathrm{C}\left(63^{\prime}\right)-\mathrm{C}\left(64^{\prime}\right)$ & $1.377(5)$ & $\mathrm{N}(2) \# 1-\mathrm{Al}(1)-\mathrm{N}(1)$ & $89.77(5)$ \\
\hline$C\left(65^{\prime}\right)-C\left(66^{\prime}\right)$ & $1.499(7)$ & $\mathrm{N}(2)-\mathrm{Al}(1)-\mathrm{N}(1)$ & $90.23(5)$ \\
\hline $\mathrm{C}(67)-\mathrm{C}(68)$ & $1.501(4)$ & $\mathrm{N}(2) \# 1-\mathrm{Al}(1)-\mathrm{N}(1) \# 1$ & $90.23(5)$ \\
\hline $\mathrm{C}(69)-\mathrm{C}(70)$ & $1.519(3)$ & $\mathrm{N}(2)-\mathrm{Al}(1)-\mathrm{N}(1) \# 1$ & $89.77(5)$ \\
\hline$C(71)-C(72)$ & $1.495(4)$ & $\mathrm{N}(1)-\mathrm{Al}(1)-\mathrm{N}(1) \# 1$ & 180.0 \\
\hline$C(73)-C(74)$ & $1.509(7)$ & $\mathrm{N}(2) \# 1-\mathrm{Al}(1)-\mathrm{O}(1)$ & $90.49(5)$ \\
\hline$C\left(73^{\prime}\right)-C\left(74^{\prime}\right)$ & $1.422(9)$ & $\mathrm{N}(2)-\mathrm{Al}(1)-\mathrm{O}(1)$ & $89.51(5)$ \\
\hline$C(75)-C(76)$ & $1.500(4)$ & $\mathrm{N}(1)-\mathrm{Al}(1)-\mathrm{O}(1)$ & $88.43(5)$ \\
\hline $\mathrm{C}(77)-\mathrm{C}(78)$ & $1.520(3)$ & $\mathrm{N}(1) \# 1-\mathrm{Al}(1)-\mathrm{O}(1)$ & $91.57(5)$ \\
\hline$C(79)-C(80)$ & $1.507(4)$ & $\mathrm{N}(2) \# 1-\mathrm{Al}(1)-\mathrm{O}(1) \# 1$ & $89.51(5)$ \\
\hline $\mathrm{C}(81)-\mathrm{C}(82)$ & $1.510(7)$ & $\mathrm{N}(2)-\mathrm{Al}(1)-\mathrm{O}(1) \# 1$ & $90.49(5)$ \\
\hline$C(82)-C(83)$ & $1.511(8)$ & $\mathrm{N}(1)-\mathrm{Al}(1)-\mathrm{O}(1) \# 1$ & $91.57(5)$ \\
\hline $\mathrm{C}(83)-\mathrm{C}(84)$ & $1.498(9)$ & $\mathrm{N}(1) \# 1-\mathrm{Al}(1)-\mathrm{O}(1) \# 1$ & $88.43(5)$ \\
\hline C(81')-C(82') & $1.447(8)$ & $\mathrm{O}(1)-\mathrm{Al}(1)-\mathrm{O}(1) \# 1$ & $180.00(5)$ \\
\hline$C\left(82^{\prime}\right)-C\left(83^{\prime}\right)$ & $1.448(12)$ & $\mathrm{N}(3)-\mathrm{Al}(2)-\mathrm{N}(3) \# 2$ & 180.0 \\
\hline $\mathrm{C}\left(83^{\prime}\right)-\mathrm{C}\left(84^{\prime}\right)$ & $1.505(11)$ & $\mathrm{N}(3)-\mathrm{Al}(2)-\mathrm{N}(4) \# 2$ & $90.59(5)$ \\
\hline$C(85)-C(86)$ & $1.554(9)$ & $\mathrm{N}(3) \# 2-\mathrm{Al}(2)-\mathrm{N}(4) \# 2$ & $89.41(5)$ \\
\hline$C(86)-C(87)$ & $1.478(8)$ & N(3)-Al(2)-N(4) & $89.41(5)$ \\
\hline C(87)-C(88) & $1.503(8)$ & $\mathrm{N}(3) \# 2-\mathrm{Al}(2)-\mathrm{N}(4)$ & $90.59(5)$ \\
\hline $\mathrm{C}\left(85^{\prime}\right)-\mathrm{C}\left(86^{\prime}\right)$ & $1.490(8)$ & $\mathrm{N}(4) \# 2-\mathrm{Al}(2)-\mathrm{N}(4)$ & 180.0 \\
\hline$C\left(86^{\prime}\right)-C\left(87^{\prime}\right)$ & $1.374(10)$ & $\mathrm{N}(3)-\mathrm{Al}(2)-\mathrm{O}(2) \# 2$ & $90.21(5)$ \\
\hline \multirow[t]{2}{*}{$\mathrm{C}\left(87^{\prime}\right)-\mathrm{C}\left(88^{\prime}\right)$} & $1.479(8)$ & $\mathrm{N}(3) \# 2-\mathrm{Al}(2)-\mathrm{O}(2) \# 2$ & $89.79(5)$ \\
\hline & & $\mathrm{N}(4) \# 2-\mathrm{Al}(2)-\mathrm{O}(2) \# 2$ & $90.46(5)$ \\
\hline C(91)-Co(1)-C(89) & $109.79(10)$ & $\mathrm{N}(4)-\mathrm{Al}(2)-\mathrm{O}(2) \# 2$ & $89.54(5)$ \\
\hline C(91)-Co(1)-C(90) & $109.65(12)$ & $\mathrm{N}(3)-\mathrm{Al}(2)-\mathrm{O}(2)$ & $89.79(5)$ \\
\hline C(89)-Co(1)-C(90) & $107.37(11)$ & $\mathrm{N}(3) \# 2-\mathrm{Al}(2)-\mathrm{O}(2)$ & $90.21(5)$ \\
\hline $\mathrm{C}(91)-\mathrm{Co}(1)-\mathrm{C}(92)$ & $107.70(11)$ & $\mathrm{N}(4) \# 2-\mathrm{Al}(2)-\mathrm{O}(2)$ & $89.54(5)$ \\
\hline $\mathrm{C}(89)-\mathrm{Co}(1)-\mathrm{C}(92)$ & $111.10(12)$ & $\mathrm{N}(4)-\mathrm{Al}(2)-\mathrm{O}(2)$ & $90.45(5)$ \\
\hline
\end{tabular}




\begin{tabular}{|c|c|c|c|}
\hline $\mathrm{O}(2) \# 2-\mathrm{Al}(2)-\mathrm{O}(2)$ & 180.0 & $\mathrm{C}(85)-\mathrm{O}(4)-\mathrm{C}\left(85^{\prime}\right)$ & $70.6(3)$ \\
\hline $\mathrm{N}(7)-\mathrm{Al}(3)-\mathrm{O}(4)$ & $89.64(8)$ & $\mathrm{C}\left(88^{\prime}\right)-\mathrm{O}(4)-\mathrm{C}\left(85^{\prime}\right)$ & $98.9(3)$ \\
\hline $\mathrm{N}(7)-\mathrm{Al}(3)-\mathrm{O}(3)$ & $90.03(8)$ & $\mathrm{C}(88)-\mathrm{O}(4)-\mathrm{Al}(3)$ & $120.4(2)$ \\
\hline $\mathrm{O}(4)-\mathrm{Al}(3)-\mathrm{O}(3)$ & $179.56(6)$ & $\mathrm{C}(85)-\mathrm{O}(4)-\mathrm{Al}(3)$ & $117.4(2)$ \\
\hline $\mathrm{N}(7)-\mathrm{Al}(3)-\mathrm{N}(5)$ & $179.79(10)$ & $\mathrm{C}\left(88^{\prime}\right)-\mathrm{O}(4)-\mathrm{Al}(3)$ & $128.88(19)$ \\
\hline $\mathrm{O}(4)-\mathrm{Al}(3)-\mathrm{N}(5)$ & $90.53(7)$ & $\mathrm{C}\left(85^{\prime}\right)-\mathrm{O}(4)-\mathrm{Al}(3)$ & $130.6(2)$ \\
\hline $\mathrm{O}(3)-\mathrm{Al}(3)-\mathrm{N}(5)$ & $89.80(7)$ & $\mathrm{C}(1)-\mathrm{N}(1)-\mathrm{C}(4)$ & $104.51(13)$ \\
\hline $\mathrm{N}(7)-\mathrm{Al}(3)-\mathrm{N}(8)$ & $90.07(7)$ & $\mathrm{C}(1)-\mathrm{N}(1)-\mathrm{Al}(1)$ & $127.66(11)$ \\
\hline $\mathrm{O}(4)-\mathrm{Al}(3)-\mathrm{N}(8)$ & $90.42(7)$ & $\mathrm{C}(4)-\mathrm{N}(1)-\mathrm{Al}(1)$ & $126.98(10)$ \\
\hline $\mathrm{O}(3)-\mathrm{Al}(3)-\mathrm{N}(8)$ & $89.29(7)$ & $\mathrm{C}(9)-\mathrm{N}(2)-\mathrm{C}(6)$ & $105.12(12)$ \\
\hline $\mathrm{N}(5)-\mathrm{Al}(3)-\mathrm{N}(8)$ & $90.05(6)$ & $\mathrm{C}(9)-\mathrm{N}(2)-\mathrm{Al}(1)$ & $127.74(10)$ \\
\hline $\mathrm{N}(7)-\mathrm{Al}(3)-\mathrm{N}(6)$ & $90.03(7)$ & $\mathrm{C}(6)-\mathrm{N}(2)-\mathrm{Al}(1)$ & $126.88(10)$ \\
\hline $\mathrm{O}(4)-\mathrm{Al}(3)-\mathrm{N}(6)$ & $89.50(7)$ & $\mathrm{C}(26)-\mathrm{N}(3)-\mathrm{C}(23)$ & $105.20(13)$ \\
\hline $\mathrm{O}(3)-\mathrm{Al}(3)-\mathrm{N}(6)$ & $90.79(7)$ & $\mathrm{C}(26)-\mathrm{N}(3)-\mathrm{Al}(2)$ & $127.94(11)$ \\
\hline $\mathrm{N}(5)-\mathrm{Al}(3)-\mathrm{N}(6)$ & $89.85(6)$ & $\mathrm{C}(23)-\mathrm{N}(3)-\mathrm{Al}(2)$ & $126.84(11)$ \\
\hline $\mathrm{N}(8)-\mathrm{Al}(3)-\mathrm{N}(6)$ & $179.87(8)$ & $\mathrm{C}(28)-\mathrm{N}(4)-\mathrm{C}(31)$ & $104.98(13)$ \\
\hline $\mathrm{C}(22)-\mathrm{O}(1)-\mathrm{C}(19)$ & $106.72(13)$ & $\mathrm{C}(28)-\mathrm{N}(4)-\mathrm{Al}(2)$ & $128.21(11)$ \\
\hline $\mathrm{C}(22)-\mathrm{O}(1)-\mathrm{Al}(1)$ & $124.52(11)$ & $\mathrm{C}(31)-\mathrm{N}(4)-\mathrm{Al}(2)$ & $126.79(11)$ \\
\hline $\mathrm{C}(19)-\mathrm{O}(1)-\mathrm{Al}(1)$ & $123.19(10)$ & $\mathrm{C}(48)-\mathrm{N}(5)-\mathrm{C}\left(45^{\prime}\right)$ & $106.17(18)$ \\
\hline $\mathrm{C}(41)-\mathrm{O}(2)-\mathrm{C}(44)$ & $109.42(14)$ & $\mathrm{C}(48)-\mathrm{N}(5)-\mathrm{C}(45)$ & $103.0(2)$ \\
\hline $\mathrm{C}(41)-\mathrm{O}(2)-\mathrm{Al}(2)$ & $125.52(11)$ & $\mathrm{C}\left(45^{\prime}\right)-\mathrm{N}(5)-\mathrm{C}(45)$ & $27.02(17)$ \\
\hline $\mathrm{C}(44)-\mathrm{O}(2)-\mathrm{Al}(2)$ & $124.90(10)$ & $\mathrm{C}(48)-\mathrm{N}(5)-\mathrm{Al}(3)$ & $127.52(12)$ \\
\hline $\mathrm{C}\left(81^{\prime}\right)-\mathrm{O}(3)-\mathrm{C}\left(84^{\prime}\right)$ & $120.1(3)$ & $\mathrm{C}\left(45^{\prime}\right)-\mathrm{N}(5)-\mathrm{Al}(3)$ & $125.49(17)$ \\
\hline $\mathrm{C}\left(81^{\prime}\right)-\mathrm{O}(3)-\mathrm{C}(84)$ & $79.3(4)$ & $\mathrm{C}(45)-\mathrm{N}(5)-\mathrm{Al}(3)$ & $126.77(19)$ \\
\hline $\mathrm{C}\left(84^{\prime}\right)-\mathrm{O}(3)-\mathrm{C}(84)$ & $55.5(3)$ & $\mathrm{C}(50)-\mathrm{N}(6)-\mathrm{C}(53)$ & $104.52(15)$ \\
\hline $\mathrm{C}\left(81^{\prime}\right)-\mathrm{O}(3)-\mathrm{C}(81)$ & $54.2(4)$ & $\mathrm{C}(50)-\mathrm{N}(6)-\mathrm{Al}(3)$ & $127.60(12)$ \\
\hline $\mathrm{C}\left(84^{\prime}\right)-\mathrm{O}(3)-\mathrm{C}(81)$ & $92.9(3)$ & $\mathrm{C}(53)-\mathrm{N}(6)-\mathrm{Al}(3)$ & $127.86(13)$ \\
\hline $\mathrm{C}(84)-\mathrm{O}(3)-\mathrm{C}(81)$ & $98.7(2)$ & $\mathrm{C}\left(55^{\prime}\right)-\mathrm{N}(7)-\mathrm{C}(58)$ & $103.2(2)$ \\
\hline $\mathrm{C}\left(81^{\prime}\right)-\mathrm{O}(3)-\mathrm{Al}(3)$ & $121.2(2)$ & $\mathrm{C}\left(55^{\prime}\right)-\mathrm{N}(7)-\mathrm{C}(55)$ & $26.76(19)$ \\
\hline $\mathrm{C}\left(84^{\prime}\right)-\mathrm{O}(3)-\mathrm{Al}(3)$ & $118.1(2)$ & $\mathrm{C}(58)-\mathrm{N}(7)-\mathrm{C}(55)$ & $105.70(19)$ \\
\hline $\mathrm{C}(84)-\mathrm{O}(3)-\mathrm{Al}(3)$ & $130.99(18)$ & $\mathrm{C}\left(55^{\prime}\right)-\mathrm{N}(7)-\mathrm{Al}(3)$ & $127.6(2)$ \\
\hline $\mathrm{C}(81)-\mathrm{O}(3)-\mathrm{Al}(3)$ & $129.96(18)$ & $\mathrm{C}(58)-\mathrm{N}(7)-\mathrm{Al}(3)$ & $127.51(12)$ \\
\hline C(88)-O(4)-C(85) & $120.8(3)$ & $\mathrm{C}(55)-\mathrm{N}(7)-\mathrm{Al}(3)$ & $125.33(17)$ \\
\hline $\mathrm{C}(88)-\mathrm{O}(4)-\mathrm{C}\left(88^{\prime}\right)$ & $65.9(4)$ & $\mathrm{C}\left(63^{\prime}\right)-\mathrm{N}(8)-\mathrm{C}(60)$ & $104.2(2)$ \\
\hline $\mathrm{C}(85)-\mathrm{O}(4)-\mathrm{C}\left(88^{\prime}\right)$ & $87.4(4)$ & $\mathrm{C}\left(63^{\prime}\right)-\mathrm{N}(8)-\mathrm{C}(63)$ & $21.74(19)$ \\
\hline $\mathrm{C}(88)-\mathrm{O}(4)-\mathrm{C}\left(85^{\prime}\right)$ & $63.7(4)$ & $\mathrm{C}(60)-\mathrm{N}(8)-\mathrm{C}(63)$ & $104.6(2)$ \\
\hline
\end{tabular}




\begin{tabular}{|c|c|c|c|}
\hline $\mathrm{C}\left(63^{\prime}\right)-\mathrm{N}(8)-\mathrm{Al}(3)$ & $127.9(2)$ & $\mathrm{O}(1)-\mathrm{C}(22)-\mathrm{C}(21)$ & $106.60(16)$ \\
\hline $\mathrm{C}(60)-\mathrm{N}(8)-\mathrm{Al}(3)$ & $127.47(12)$ & $\mathrm{N}(3)-\mathrm{C}(23)-\mathrm{C}(32) \# 2$ & $124.88(14)$ \\
\hline $\mathrm{C}(63)-\mathrm{N}(8)-\mathrm{Al}(3)$ & $125.8(2)$ & $\mathrm{N}(3)-\mathrm{C}(23)-\mathrm{C}(24)$ & $110.78(14)$ \\
\hline $\mathrm{N}(1)-\mathrm{C}(1)-\mathrm{C}(10)$ & $124.35(14)$ & $\mathrm{C}(32) \# 2-\mathrm{C}(23)-\mathrm{C}(24)$ & $124.34(15)$ \\
\hline $\mathrm{N}(1)-\mathrm{C}(1)-\mathrm{C}(2)$ & $111.02(14)$ & $C(25)-C(24)-C(23)$ & $106.68(15)$ \\
\hline$C(10)-C(1)-C(2)$ & $124.48(15)$ & $\mathrm{C}(25)-\mathrm{C}(24)-\mathrm{C}(33)$ & $127.63(16)$ \\
\hline$C(3)-C(2)-C(1)$ & $106.80(14)$ & $\mathrm{C}(23)-\mathrm{C}(24)-\mathrm{C}(33)$ & $125.69(16)$ \\
\hline$C(3)-C(2)-C(11)$ & $128.39(15)$ & $C(24)-C(25)-C(26)$ & $106.79(15)$ \\
\hline$C(1)-C(2)-C(11)$ & $124.79(15)$ & $C(24)-C(25)-C(35)$ & $127.47(16)$ \\
\hline$C(2)-C(3)-C(4)$ & $106.29(14)$ & $C(26)-C(25)-C(35)$ & $125.71(16)$ \\
\hline$C(2)-C(3)-C(13)$ & $127.49(15)$ & $N(3)-C(26)-C(27)$ & $124.71(15)$ \\
\hline$C(4)-C(3)-C(13)$ & $126.16(15)$ & $\mathrm{N}(3)-\mathrm{C}(26)-\mathrm{C}(25)$ & $110.51(14)$ \\
\hline $\mathrm{C}(5)-\mathrm{C}(4)-\mathrm{N}(1)$ & $124.27(14)$ & $\mathrm{C}(27)-\mathrm{C}(26)-\mathrm{C}(25)$ & $124.71(15)$ \\
\hline$C(5)-C(4)-C(3)$ & $124.35(15)$ & $\mathrm{C}(26)-\mathrm{C}(27)-\mathrm{C}(28)$ & $125.28(15)$ \\
\hline $\mathrm{N}(1)-\mathrm{C}(4)-\mathrm{C}(3)$ & $111.37(14)$ & $\mathrm{N}(4)-\mathrm{C}(28)-\mathrm{C}(27)$ & $124.36(15)$ \\
\hline$C(4)-C(5)-C(6)$ & $126.08(15)$ & $\mathrm{N}(4)-\mathrm{C}(28)-\mathrm{C}(29)$ & $110.87(14)$ \\
\hline $\mathrm{C}(5)-\mathrm{C}(6)-\mathrm{N}(2)$ & $124.84(14)$ & $\mathrm{C}(27)-\mathrm{C}(28)-\mathrm{C}(29)$ & $124.68(15)$ \\
\hline$C(5)-C(6)-C(7)$ & $124.34(14)$ & $\mathrm{C}(30)-\mathrm{C}(29)-\mathrm{C}(28)$ & $106.72(14)$ \\
\hline $\mathrm{N}(2)-\mathrm{C}(6)-\mathrm{C}(7)$ & $110.76(13)$ & $\mathrm{C}(30)-\mathrm{C}(29)-\mathrm{C}(37)$ & $128.67(16)$ \\
\hline$C(8)-C(7)-C(6)$ & $106.67(14)$ & $\mathrm{C}(28)-\mathrm{C}(29)-\mathrm{C}(37)$ & $124.50(15)$ \\
\hline $\mathrm{C}(8)-\mathrm{C}(7)-\mathrm{C}(15)$ & $128.33(15)$ & $\mathrm{C}(29)-\mathrm{C}(30)-\mathrm{C}(31)$ & $106.74(14)$ \\
\hline$C(6)-C(7)-C(15)$ & $125.00(15)$ & $\mathrm{C}(29)-\mathrm{C}(30)-\mathrm{C}(39)$ & $128.72(16)$ \\
\hline $\mathrm{C}(7)-\mathrm{C}(8)-\mathrm{C}(9)$ & $106.67(14)$ & $\mathrm{C}(31)-\mathrm{C}(30)-\mathrm{C}(39)$ & $124.54(16)$ \\
\hline$C(7)-C(8)-C(17)$ & $128.33(15)$ & $\mathrm{C}(32)-\mathrm{C}(31)-\mathrm{N}(4)$ & $124.96(15)$ \\
\hline$C(9)-C(8)-C(17)$ & $124.99(15)$ & $\mathrm{C}(32)-\mathrm{C}(31)-\mathrm{C}(30)$ & $124.38(15)$ \\
\hline $\mathrm{N}(2)-\mathrm{C}(9)-\mathrm{C}(10) \# 1$ & $124.63(14)$ & $\mathrm{N}(4)-\mathrm{C}(31)-\mathrm{C}(30)$ & $110.67(14)$ \\
\hline $\mathrm{N}(2)-\mathrm{C}(9)-\mathrm{C}(8)$ & $110.76(13)$ & $\mathrm{C}(31)-\mathrm{C}(32)-\mathrm{C}(23) \# 2$ & $125.75(15)$ \\
\hline $\mathrm{C}(10) \# 1-\mathrm{C}(9)-\mathrm{C}(8)$ & $124.58(14)$ & $\mathrm{C}(24)-\mathrm{C}(33)-\mathrm{C}(34)$ & $113.23(17)$ \\
\hline$C(9) \# 1-C(10)-C(1)$ & $125.69(15)$ & $\mathrm{C}(25)-\mathrm{C}(35)-\mathrm{C}(36)$ & $112.82(17)$ \\
\hline $\mathrm{C}(2)-\mathrm{C}(11)-\mathrm{C}(12)$ & $112.77(16)$ & $\mathrm{C}(38)-\mathrm{C}(37)-\mathrm{C}(29)$ & $111.27(16)$ \\
\hline$C(14)-C(13)-C(3)$ & $114.83(17)$ & $C(30)-C(39)-C(40)$ & $114.42(17)$ \\
\hline$C(7)-C(15)-C(16)$ & $112.91(16)$ & $\mathrm{O}(2)-\mathrm{C}(41)-\mathrm{C}(42)$ & $106.97(17)$ \\
\hline$C(8)-C(17)-C(18)$ & $113.12(15)$ & $\mathrm{C}(41)-\mathrm{C}(42)-\mathrm{C}(43)$ & $105.38(19)$ \\
\hline $\mathrm{O}(1)-\mathrm{C}(19)-\mathrm{C}(20)$ & $105.46(14)$ & $\mathrm{C}(42)-\mathrm{C}(43)-\mathrm{C}(44)$ & 104.51(16) \\
\hline$C(21)-C(20)-C(19)$ & $105.72(16)$ & $\mathrm{O}(2)-\mathrm{C}(44)-\mathrm{C}(43)$ & $106.10(14)$ \\
\hline$C(22)-C(21)-C(20)$ & $107.25(17)$ & $\mathrm{C}(64)-\mathrm{C}(45)-\mathrm{N}(5)$ & $122.7(3)$ \\
\hline
\end{tabular}




\begin{tabular}{|c|c|c|c|}
\hline$C(64)-C(45)-C(46)$ & $125.6(4)$ & $\mathrm{C}(55)-\mathrm{C}(54)-\mathrm{C}(53)$ & $124.7(3)$ \\
\hline $\mathrm{N}(5)-\mathrm{C}(45)-\mathrm{C}(46)$ & $110.8(3)$ & $C(54)-C(55)-C(56)$ & $125.4(3)$ \\
\hline $\mathrm{C}(47)-\mathrm{C}(46)-\mathrm{C}(45)$ & $105.4(3)$ & $\mathrm{C}(54)-\mathrm{C}(55)-\mathrm{N}(7)$ & $124.0(3)$ \\
\hline$C(47)-C(46)-C(65)$ & $128.0(3)$ & $\mathrm{C}(56)-\mathrm{C}(55)-\mathrm{N}(7)$ & $109.7(3)$ \\
\hline$C(45)-C(46)-C(65)$ & $125.9(4)$ & $C(57)-C(56)-C(55)$ & $106.2(3)$ \\
\hline $\mathrm{C}\left(64^{\prime}\right)-\mathrm{C}\left(45^{\prime}\right)-\mathrm{N}(5)$ & $124.9(3)$ & $C(57)-C(56)-C(73)$ & $127.1(3)$ \\
\hline$C\left(64^{\prime}\right)-C\left(45^{\prime}\right)-C\left(46^{\prime}\right)$ & $125.0(3)$ & $C(55)-C(56)-C(73)$ & $126.3(3)$ \\
\hline $\mathrm{N}(5)-\mathrm{C}\left(45^{\prime}\right)-\mathrm{C}\left(46^{\prime}\right)$ & $109.4(3)$ & $\mathrm{C}\left(55^{\prime}\right)-\mathrm{C}\left(54^{\prime}\right)-\mathrm{C}(53)$ & $124.8(4)$ \\
\hline $\mathrm{C}(47)-\mathrm{C}\left(46^{\prime}\right)-\mathrm{C}\left(45^{\prime}\right)$ & $107.0(3)$ & $\mathrm{N}(7)-\mathrm{C}\left(55^{\prime}\right)-\mathrm{C}\left(54^{\prime}\right)$ & $123.3(4)$ \\
\hline$C(47)-C\left(46^{\prime}\right)-C\left(65^{\prime}\right)$ & $127.0(3)$ & $\mathrm{N}(7)-\mathrm{C}\left(55^{\prime}\right)-\mathrm{C}\left(56^{\prime}\right)$ & $110.1(4)$ \\
\hline $\mathrm{C}\left(45^{\prime}\right)-\mathrm{C}\left(46^{\prime}\right)-\mathrm{C}\left(65^{\prime}\right)$ & $125.2(3)$ & $\mathrm{C}\left(54^{\prime}\right)-\mathrm{C}\left(55^{\prime}\right)-\mathrm{C}\left(56^{\prime}\right)$ & $125.4(4)$ \\
\hline$C(46)-C(47)-C\left(46^{\prime}\right)$ & $31.05(19)$ & $\mathrm{C}(57)-\mathrm{C}\left(56^{\prime}\right)-\mathrm{C}\left(55^{\prime}\right)$ & $107.5(4)$ \\
\hline $\mathrm{C}(46)-\mathrm{C}(47)-\mathrm{C}(48)$ & $107.1(2)$ & $\mathrm{C}(57)-\mathrm{C}\left(56^{\prime}\right)-\mathrm{C}\left(73^{\prime}\right)$ & $126.5(4)$ \\
\hline$C\left(46^{\prime}\right)-C(47)-C(48)$ & $104.46(19)$ & $\mathrm{C}\left(55^{\prime}\right)-\mathrm{C}\left(56^{\prime}\right)-\mathrm{C}\left(73^{\prime}\right)$ & $124.1(4)$ \\
\hline $\mathrm{C}(46)-\mathrm{C}(47)-\mathrm{C}(67)$ & $124.2(2)$ & $\mathrm{C}\left(56^{\prime}\right)-\mathrm{C}(57)-\mathrm{C}(56)$ & $29.6(2)$ \\
\hline $\mathrm{C}\left(46^{\prime}\right)-\mathrm{C}(47)-\mathrm{C}(67)$ & $128.7(2)$ & $\mathrm{C}\left(56^{\prime}\right)-\mathrm{C}(57)-\mathrm{C}(58)$ & $104.8(2)$ \\
\hline $\mathrm{C}(48)-\mathrm{C}(47)-\mathrm{C}(67)$ & $125.48(18)$ & $\mathrm{C}(56)-\mathrm{C}(57)-\mathrm{C}(58)$ & $106.4(2)$ \\
\hline $\mathrm{N}(5)-\mathrm{C}(48)-\mathrm{C}(49)$ & $124.82(15)$ & $\mathrm{C}\left(56^{\prime}\right)-\mathrm{C}(57)-\mathrm{C}(75)$ & $126.9(3)$ \\
\hline $\mathrm{N}(5)-\mathrm{C}(48)-\mathrm{C}(47)$ & $111.02(15)$ & $\mathrm{C}(56)-\mathrm{C}(57)-\mathrm{C}(75)$ & $127.2(2)$ \\
\hline $\mathrm{C}(49)-\mathrm{C}(48)-\mathrm{C}(47)$ & $124.16(16)$ & $\mathrm{C}(58)-\mathrm{C}(57)-\mathrm{C}(75)$ & $125.2(2)$ \\
\hline $\mathrm{C}(50)-\mathrm{C}(49)-\mathrm{C}(48)$ & $125.43(16)$ & $\mathrm{N}(7)-\mathrm{C}(58)-\mathrm{C}(59)$ & $124.76(16)$ \\
\hline $\mathrm{N}(6)-\mathrm{C}(50)-\mathrm{C}(49)$ & $124.71(15)$ & $\mathrm{N}(7)-\mathrm{C}(58)-\mathrm{C}(57)$ & $110.95(17)$ \\
\hline $\mathrm{N}(6)-\mathrm{C}(50)-\mathrm{C}(51)$ & $111.26(15)$ & $\mathrm{C}(59)-\mathrm{C}(58)-\mathrm{C}(57)$ & $124.28(17)$ \\
\hline $\mathrm{C}(49)-\mathrm{C}(50)-\mathrm{C}(51)$ & $124.03(16)$ & $\mathrm{C}(58)-\mathrm{C}(59)-\mathrm{C}(60)$ & $125.46(17)$ \\
\hline $\mathrm{C}(52)-\mathrm{C}(51)-\mathrm{C}(50)$ & $106.55(16)$ & $\mathrm{N}(8)-\mathrm{C}(60)-\mathrm{C}(59)$ & $124.57(16)$ \\
\hline $\mathrm{C}(52)-\mathrm{C}(51)-\mathrm{C}(69)$ & $127.95(17)$ & $\mathrm{N}(8)-\mathrm{C}(60)-\mathrm{C}(61)$ & $111.16(15)$ \\
\hline $\mathrm{C}(50)-\mathrm{C}(51)-\mathrm{C}(69)$ & $125.47(16)$ & $C(59)-C(60)-C(61)$ & $124.27(16)$ \\
\hline $\mathrm{C}(51)-\mathrm{C}(52)-\mathrm{C}(53)$ & $106.61(16)$ & $\mathrm{C}(62)-\mathrm{C}(61)-\mathrm{C}(60)$ & $106.45(16)$ \\
\hline $\mathrm{C}(51)-\mathrm{C}(52)-\mathrm{C}(71)$ & $127.67(18)$ & $\mathrm{C}(62)-\mathrm{C}(61)-\mathrm{C}(77)$ & $128.01(17)$ \\
\hline $\mathrm{C}(53)-\mathrm{C}(52)-\mathrm{C}(71)$ & $125.68(19)$ & $\mathrm{C}(60)-\mathrm{C}(61)-\mathrm{C}(77)$ & $125.53(16)$ \\
\hline $\mathrm{N}(6)-\mathrm{C}(53)-\mathrm{C}(54)$ & $123.7(2)$ & $\mathrm{C}(61)-\mathrm{C}(62)-\mathrm{C}\left(63^{\prime}\right)$ & $105.2(2)$ \\
\hline $\mathrm{N}(6)-\mathrm{C}(53)-\mathrm{C}(52)$ & $111.04(17)$ & $\mathrm{C}(61)-\mathrm{C}(62)-\mathrm{C}(63)$ & $107.7(2)$ \\
\hline $\mathrm{C}(54)-\mathrm{C}(53)-\mathrm{C}(52)$ & $123.2(2)$ & $\mathrm{C}\left(63^{\prime}\right)-\mathrm{C}(62)-\mathrm{C}(63)$ & $20.98(18)$ \\
\hline $\mathrm{N}(6)-\mathrm{C}(53)-\mathrm{C}\left(54^{\prime}\right)$ & $120.8(2)$ & $\mathrm{C}(61)-\mathrm{C}(62)-\mathrm{C}(79)$ & $127.97(18)$ \\
\hline $\mathrm{C}(54)-\mathrm{C}(53)-\mathrm{C}\left(54^{\prime}\right)$ & 29.31(19) & $C\left(63^{\prime}\right)-C(62)-C(79)$ & $126.0(2)$ \\
\hline $\mathrm{C}(52)-\mathrm{C}(53)-\mathrm{C}\left(54^{\prime}\right)$ & $125.1(2)$ & $\mathrm{C}(63)-\mathrm{C}(62)-\mathrm{C}(79)$ & $123.3(3)$ \\
\hline
\end{tabular}




$\begin{array}{lllr}\mathrm{C}(64)-\mathrm{C}(63)-\mathrm{N}(8) & 124.4(4) & \mathrm{C}(83)-\mathrm{C}(84)-\mathrm{O}(3) & 106.3(3) \\ \mathrm{C}(64)-\mathrm{C}(63)-\mathrm{C}(62) & 127.7(4) & \mathrm{O}(3)-\mathrm{C}\left(81^{\prime}\right)-\mathrm{C}\left(82^{\prime}\right) & 100.8(4) \\ \mathrm{N}(8)-\mathrm{C}(63)-\mathrm{C}(62) & 105.8(3) & \mathrm{C}\left(83^{\prime}\right)-\mathrm{C}\left(82^{\prime}\right)-\mathrm{C}\left(81^{\prime}\right) & 107.5(6) \\ \mathrm{C}(63)-\mathrm{C}(64)-\mathrm{C}(45) & 126.4(4) & \mathrm{C}\left(82^{\prime}\right)-\mathrm{C}\left(83^{\prime}\right)-\mathrm{C}\left(84^{\prime}\right) & 106.4(7) \\ \mathrm{C}(46)-\mathrm{C}(65)-\mathrm{C}(66) & 114.5(4) & \mathrm{O}(3)-\mathrm{C}\left(84^{\prime}\right)-\mathrm{C}\left(83^{\prime}\right) & 99.3(5) \\ \mathrm{N}(8)-\mathrm{C}\left(63^{\prime}\right)-\mathrm{C}\left(64^{\prime}\right) & 124.0(4) & \mathrm{O}(4)-\mathrm{C}(85)-\mathrm{C}(86) & 98.3(4) \\ \mathrm{N}(8)-\mathrm{C}\left(63^{\prime}\right)-\mathrm{C}(62) & 110.5(3) & \mathrm{C}(87)-\mathrm{C}(86)-\mathrm{C}(85) & 102.1(5) \\ \mathrm{C}\left(64^{\prime}\right)-\mathrm{C}\left(63^{\prime}\right)-\mathrm{C}(62) & 122.9(3) & \mathrm{C}(86)-\mathrm{C}(87)-\mathrm{C}(88) & 107.5(5) \\ \mathrm{C}\left(63^{\prime}\right)-\mathrm{C}\left(64^{\prime}\right)-\mathrm{C}\left(45^{\prime}\right) & 126.0(3) & \mathrm{O}(4)-\mathrm{C}(88)-\mathrm{C}(87) & 100.7(4) \\ \mathrm{C}\left(66^{\prime}\right)-\mathrm{C}\left(65^{\prime}\right)-\mathrm{C}\left(46^{\prime}\right) & 112.5(4) & \mathrm{C}\left(86^{\prime}\right)-\mathrm{C}\left(85^{\prime}\right)-\mathrm{O}(4) & 107.3(4) \\ \mathrm{C}(47)-\mathrm{C}(67)-\mathrm{C}(68) & 113.3(2) & \mathrm{C}\left(87^{\prime}\right)-\mathrm{C}\left(86^{\prime}\right)-\mathrm{C}\left(85^{\prime}\right) & 106.2(6) \\ \mathrm{C}(51)-\mathrm{C}(69)-\mathrm{C}(70) & 113.33(18) & \mathrm{C}\left(86^{\prime}\right)-\mathrm{C}\left(87^{\prime}\right)-\mathrm{C}\left(88^{\prime}\right) & 110.3(6) \\ \mathrm{C}(72)-\mathrm{C}(71)-\mathrm{C}(52) & 112.8(2) & \mathrm{C}\left(87^{\prime}\right)-\mathrm{C}\left(88^{\prime}\right)-\mathrm{O}(4) & 108.3(4) \\ \mathrm{C}(56)-\mathrm{C}(73)-\mathrm{C}(74) & 114.8(4) & \mathrm{O}(5)-\mathrm{C}(89)-\mathrm{Co}(1) & 178.5(2) \\ \mathrm{C}\left(74^{\prime}\right)-\mathrm{C}\left(73^{\prime}\right)-\mathrm{C}\left(56^{\prime}\right) & 111.4(5) & \mathrm{O}(6)-\mathrm{C}(90)-\mathrm{Co}(1) & 177.7(3) \\ \mathrm{C}(76)-\mathrm{C}(75)-\mathrm{C}(57) & 113.2(2) & \mathrm{O}(7)-\mathrm{C}(91)-\mathrm{Co}(1) & 179.4(2) \\ \mathrm{C}(61)-\mathrm{C}(77)-\mathrm{C}(78) & 113.54(19) & \mathrm{O}(8)-\mathrm{C}(92)-\mathrm{Co}(1) & 179.1(3) \\ \mathrm{C}(62)-\mathrm{C}(79)-\mathrm{C}(80) & 112.5(2) & \mathrm{O}(9)-\mathrm{C}(93)-\mathrm{Co}(2) & 178.2(2) \\ \mathrm{C}(82)-\mathrm{C}(81)-\mathrm{O}(3) & 110.8(3) & \mathrm{O}(10)-\mathrm{C}(94)-\mathrm{Co}(2) & 178.9(3) \\ \mathrm{C}(81)-\mathrm{C}(82)-\mathrm{C}(83) & 103.3(4) & \mathrm{O}(11)-\mathrm{C}(95)-\mathrm{Co}(2) & 178.1(3) \\ \mathrm{C}(84)-\mathrm{C}(83)-\mathrm{C}(82) & 102.8(5) & \mathrm{O}(12)-\mathrm{C}(96)-\mathrm{Co}(2) & 179.0(3) \\ & & & \end{array}$

Symmetry transformations used to generate equivalent atoms:

$\# 1-\mathrm{x}+1,-\mathrm{y}+1,-\mathrm{z}+2 \quad \# 2-\mathrm{x},-\mathrm{y},-\mathrm{z}$ 
Table S14. Anisotropic displacement parameters $\left(\AA^{2} \times 10^{3}\right)$ for $\left[(\mathrm{OEP}) \mathrm{Al}(\mathrm{THF})_{2}\right]^{+}\left[\mathrm{Co}(\mathrm{CO})_{4}\right]^{-}$. The anisotropic displacement factor exponent takes the form: $-2 \pi^{2}\left[h^{2} a^{* 2} U^{11}+\ldots+2 h k a^{*} b^{*} U^{12}\right]$

\begin{tabular}{|c|c|c|c|c|c|c|}
\hline & $\mathrm{U}^{11}$ & $\mathrm{U}^{22}$ & $\mathrm{U}^{33}$ & $\mathrm{U}^{23}$ & $\mathrm{U}^{13}$ & $\mathrm{U}^{12}$ \\
\hline $\operatorname{Co}(1)$ & $37(1)$ & $40(1)$ & $37(1)$ & $-9(1)$ & $1(1)$ & $-10(1)$ \\
\hline $\mathrm{Co}(2)$ & $35(1)$ & $58(1)$ & $35(1)$ & $-17(1)$ & $3(1)$ & $-2(1)$ \\
\hline $\mathrm{Al}(1)$ & 21(1) & $16(1)$ & $18(1)$ & $-5(1)$ & $3(1)$ & $-5(1)$ \\
\hline $\mathrm{Al}(2)$ & $23(1)$ & $16(1)$ & $17(1)$ & $-5(1)$ & $3(1)$ & $-5(1)$ \\
\hline $\operatorname{Al}(3)$ & $31(1)$ & $59(1)$ & $27(1)$ & $-26(1)$ & $14(1)$ & $-29(1)$ \\
\hline $\mathrm{O}(1)$ & $23(1)$ & $25(1)$ & $20(1)$ & $-6(1)$ & $2(1)$ & $-7(1)$ \\
\hline $\mathrm{O}(2)$ & $26(1)$ & $20(1)$ & $25(1)$ & $-1(1)$ & $-2(1)$ & $-7(1)$ \\
\hline $\mathrm{O}(3)$ & $33(1)$ & 61(1) & $39(1)$ & $-28(1)$ & $9(1)$ & $-23(1)$ \\
\hline $\mathrm{O}(4)$ & $37(1)$ & $63(1)$ & $30(1)$ & $-21(1)$ & $8(1)$ & $-25(1)$ \\
\hline $\mathrm{O}(5)$ & $53(1)$ & $77(1)$ & $67(1)$ & $-38(1)$ & $15(1)$ & $-6(1)$ \\
\hline $\mathrm{O}(6)$ & $72(2)$ & $48(1)$ & $170(3)$ & $-24(1)$ & $-11(2)$ & $-17(1)$ \\
\hline $\mathrm{O}(7)$ & $69(1)$ & $46(1)$ & $48(1)$ & $-10(1)$ & $17(1)$ & $-12(1)$ \\
\hline $\mathrm{O}(8)$ & $65(1)$ & $129(2)$ & $63(1)$ & $-12(1)$ & $-16(1)$ & $-41(1)$ \\
\hline $\mathrm{O}(9)$ & $58(1)$ & $117(2)$ & $77(2)$ & $-8(1)$ & $-21(1)$ & $-30(1)$ \\
\hline $\mathrm{O}(10)$ & $53(1)$ & $98(2)$ & $59(1)$ & $-43(1)$ & $10(1)$ & $4(1)$ \\
\hline $\mathrm{O}(11)$ & $92(2)$ & $70(1)$ & $74(1)$ & $-24(1)$ & $13(1)$ & $-28(1)$ \\
\hline $\mathrm{O}(12)$ & $87(2)$ & $67(1)$ & $49(1)$ & $-11(1)$ & $5(1)$ & $-21(1)$ \\
\hline $\mathrm{N}(1)$ & $22(1)$ & $19(1)$ & $20(1)$ & $-6(1)$ & $4(1)$ & $-4(1)$ \\
\hline $\mathrm{N}(2)$ & $23(1)$ & $18(1)$ & $20(1)$ & $-7(1)$ & $3(1)$ & $-5(1)$ \\
\hline $\mathrm{N}(3)$ & $26(1)$ & $20(1)$ & $19(1)$ & $-6(1)$ & $4(1)$ & $-8(1)$ \\
\hline $\mathrm{N}(4)$ & $26(1)$ & $18(1)$ & $22(1)$ & $-7(1)$ & $3(1)$ & $-6(1)$ \\
\hline $\mathrm{N}(5)$ & $34(1)$ & $55(1)$ & $31(1)$ & $-27(1)$ & $16(1)$ & $-29(1)$ \\
\hline $\mathrm{N}(6)$ & $34(1)$ & $50(1)$ & $25(1)$ & $-18(1)$ & $10(1)$ & $-26(1)$ \\
\hline $\mathrm{N}(7)$ & $33(1)$ & $73(1)$ & $29(1)$ & $-29(1)$ & $14(1)$ & $-31(1)$ \\
\hline $\mathrm{N}(8)$ & $30(1)$ & $60(1)$ & $33(1)$ & $-31(1)$ & $14(1)$ & $-26(1)$ \\
\hline $\mathrm{C}(1)$ & $23(1)$ & $22(1)$ & $20(1)$ & $-7(1)$ & $4(1)$ & $-5(1)$ \\
\hline$C(2)$ & $25(1)$ & $24(1)$ & $22(1)$ & $-8(1)$ & $3(1)$ & $-3(1)$ \\
\hline$C(3)$ & $26(1)$ & $25(1)$ & $22(1)$ & $-9(1)$ & $2(1)$ & $-3(1)$ \\
\hline$C(4)$ & $23(1)$ & $18(1)$ & 21(1) & $-7(1)$ & $2(1)$ & $-2(1)$ \\
\hline$C(5)$ & $28(1)$ & $17(1)$ & $24(1)$ & $-8(1)$ & $2(1)$ & $-4(1)$ \\
\hline$C(6)$ & $22(1)$ & $18(1)$ & $22(1)$ & $-6(1)$ & $0(1)$ & $-4(1)$ \\
\hline
\end{tabular}




\begin{tabular}{|c|c|c|c|c|c|c|}
\hline$C(7)$ & $24(1)$ & $19(1)$ & $23(1)$ & $-5(1)$ & $-1(1)$ & $-6(1)$ \\
\hline$C(8)$ & $24(1)$ & $19(1)$ & $23(1)$ & $-4(1)$ & $1(1)$ & $-7(1)$ \\
\hline $\mathrm{C}(9)$ & $22(1)$ & $20(1)$ & $20(1)$ & $-5(1)$ & $1(1)$ & $-5(1)$ \\
\hline$C(10)$ & $25(1)$ & $22(1)$ & 21(1) & $-5(1)$ & $5(1)$ & $-7(1)$ \\
\hline $\mathrm{C}(11)$ & $32(1)$ & $30(1)$ & $23(1)$ & $-9(1)$ & $7(1)$ & $-7(1)$ \\
\hline $\mathrm{C}(12)$ & $34(1)$ & $56(1)$ & $37(1)$ & $-13(1)$ & 11(1) & $-14(1)$ \\
\hline$C(13)$ & $45(1)$ & $29(1)$ & $27(1)$ & $-14(1)$ & $8(1)$ & $-12(1)$ \\
\hline$C(14)$ & $49(1)$ & $33(1)$ & $52(1)$ & $-17(1)$ & $7(1)$ & $-1(1)$ \\
\hline$C(15)$ & $36(1)$ & $20(1)$ & $30(1)$ & $-9(1)$ & $3(1)$ & $-9(1)$ \\
\hline$C(16)$ & $53(1)$ & $24(1)$ & $49(1)$ & $-12(1)$ & $-4(1)$ & $3(1)$ \\
\hline$C(17)$ & $30(1)$ & $24(1)$ & $29(1)$ & $-6(1)$ & $6(1)$ & $-11(1)$ \\
\hline$C(18)$ & $49(1)$ & $46(1)$ & $28(1)$ & 1(1) & $3(1)$ & $-12(1)$ \\
\hline $\mathrm{C}(19)$ & $29(1)$ & $38(1)$ & 21(1) & $-3(1)$ & $0(1)$ & $-7(1)$ \\
\hline$C(20)$ & $28(1)$ & $43(1)$ & $35(1)$ & $-1(1)$ & $-4(1)$ & $-7(1)$ \\
\hline $\mathrm{C}(21)$ & $30(1)$ & $98(2)$ & $38(1)$ & $11(1)$ & $-7(1)$ & $-22(1)$ \\
\hline$C(22)$ & $27(1)$ & $49(1)$ & $37(1)$ & $4(1)$ & $-2(1)$ & $-16(1)$ \\
\hline$C(23)$ & $25(1)$ & $24(1)$ & $18(1)$ & $-6(1)$ & $2(1)$ & $-5(1)$ \\
\hline$C(24)$ & $25(1)$ & $30(1)$ & $20(1)$ & $-3(1)$ & $3(1)$ & $-5(1)$ \\
\hline$C(25)$ & $26(1)$ & $28(1)$ & $25(1)$ & $-3(1)$ & $5(1)$ & $-8(1)$ \\
\hline$C(26)$ & $26(1)$ & 21(1) & 21(1) & $-3(1)$ & $2(1)$ & $-7(1)$ \\
\hline$C(27)$ & $30(1)$ & $19(1)$ & $26(1)$ & $-4(1)$ & $1(1)$ & $-9(1)$ \\
\hline$C(28)$ & $25(1)$ & $17(1)$ & $25(1)$ & $-6(1)$ & $-2(1)$ & $-4(1)$ \\
\hline$C(29)$ & $26(1)$ & $19(1)$ & $30(1)$ & $-10(1)$ & $-5(1)$ & $-3(1)$ \\
\hline$C(30)$ & $26(1)$ & $24(1)$ & $30(1)$ & $-13(1)$ & $-2(1)$ & $-3(1)$ \\
\hline$C(31)$ & $26(1)$ & $20(1)$ & $23(1)$ & $-9(1)$ & $1(1)$ & $-3(1)$ \\
\hline$C(32)$ & $29(1)$ & $27(1)$ & $22(1)$ & $-12(1)$ & $4(1)$ & $-5(1)$ \\
\hline$C(33)$ & $39(1)$ & $37(1)$ & $22(1)$ & $-6(1)$ & $10(1)$ & $-8(1)$ \\
\hline$C(34)$ & $49(1)$ & $64(2)$ & $39(1)$ & $-14(1)$ & $13(1)$ & $9(1)$ \\
\hline$C(35)$ & $36(1)$ & $30(1)$ & $33(1)$ & $-2(1)$ & 11(1) & $-12(1)$ \\
\hline$C(36)$ & $49(1)$ & $36(1)$ & $49(1)$ & $5(1)$ & $4(1)$ & $-8(1)$ \\
\hline$C(37)$ & $31(1)$ & $23(1)$ & $37(1)$ & $-12(1)$ & $-6(1)$ & $-6(1)$ \\
\hline$C(38)$ & $71(2)$ & $27(1)$ & $79(2)$ & $-2(1)$ & $-38(2)$ & $-6(1)$ \\
\hline$C(39)$ & $37(1)$ & $31(1)$ & $35(1)$ & $-20(1)$ & $2(1)$ & $-8(1)$ \\
\hline $\mathrm{C}(40)$ & $38(1)$ & $55(1)$ & $77(2)$ & $-46(1)$ & $8(1)$ & $-7(1)$ \\
\hline $\mathrm{C}(41)$ & $41(1)$ & $41(1)$ & $58(1)$ & $18(1)$ & $-16(1)$ & $-15(1)$ \\
\hline $\mathrm{C}(42)$ & $44(1)$ & $43(1)$ & $58(2)$ & $14(1)$ & $-16(1)$ & $-12(1)$ \\
\hline
\end{tabular}




\begin{tabular}{|c|c|c|c|c|c|c|}
\hline$C(43)$ & $30(1)$ & $37(1)$ & $41(1)$ & $-4(1)$ & $-7(1)$ & $-7(1)$ \\
\hline $\mathrm{C}(44)$ & $29(1)$ & $30(1)$ & $40(1)$ & $-2(1)$ & $-3(1)$ & $-12(1)$ \\
\hline$C(45)$ & $21(2)$ & $26(2)$ & $22(2)$ & $-6(2)$ & $2(2)$ & $-4(2)$ \\
\hline$C(46)$ & $23(2)$ & $28(2)$ & $21(2)$ & $-6(2)$ & $6(1)$ & $-6(2)$ \\
\hline$C\left(45^{\prime}\right)$ & $22(2)$ & $30(2)$ & $19(2)$ & $-8(1)$ & $5(1)$ & $-10(1)$ \\
\hline $\mathrm{C}\left(46^{\prime}\right)$ & $23(2)$ & $35(2)$ & $23(2)$ & $-8(2)$ & $8(1)$ & $-9(2)$ \\
\hline $\mathrm{C}(47)$ & $48(1)$ & $63(1)$ & $40(1)$ & $-34(1)$ & $25(1)$ & $-33(1)$ \\
\hline $\mathrm{C}(48)$ & $28(1)$ & $35(1)$ & $22(1)$ & $-13(1)$ & $7(1)$ & $-11(1)$ \\
\hline C(49) & $28(1)$ & $30(1)$ & $21(1)$ & $-11(1)$ & $1(1)$ & $-7(1)$ \\
\hline $\mathrm{C}(50)$ & $26(1)$ & $27(1)$ & $21(1)$ & $-8(1)$ & $-2(1)$ & $-8(1)$ \\
\hline $\mathrm{C}(51)$ & $30(1)$ & $25(1)$ & $27(1)$ & $-8(1)$ & $-5(1)$ & $-8(1)$ \\
\hline $\mathrm{C}(52)$ & $45(1)$ & $56(1)$ & $31(1)$ & $-19(1)$ & $6(1)$ & $-33(1)$ \\
\hline $\mathrm{C}(53)$ & $55(1)$ & $93(2)$ & $35(1)$ & $-36(1)$ & $22(1)$ & $-56(1)$ \\
\hline $\mathrm{C}(54)$ & $31(2)$ & $38(2)$ & $29(2)$ & $-8(2)$ & $6(1)$ & $-18(2)$ \\
\hline$C(55)$ & $22(2)$ & $35(2)$ & $24(2)$ & $-6(1)$ & $5(1)$ & $-10(1)$ \\
\hline$C(56)$ & $26(2)$ & $36(2)$ & $23(2)$ & $-5(1)$ & $8(1)$ & $-9(1)$ \\
\hline$C\left(54^{\prime}\right)$ & $18(2)$ & $28(2)$ & $23(2)$ & $-4(2)$ & $-1(2)$ & $-11(2)$ \\
\hline$C\left(55^{\prime}\right)$ & $19(2)$ & $30(2)$ & $17(2)$ & $-7(2)$ & $0(2)$ & $-8(2)$ \\
\hline$C\left(56^{\prime}\right)$ & $24(2)$ & $31(3)$ & $28(2)$ & $-9(2)$ & $7(2)$ & $-10(2)$ \\
\hline$C(57)$ & $33(1)$ & $91(2)$ & $34(1)$ & $-34(1)$ & $16(1)$ & $-29(1)$ \\
\hline$C(58)$ & $26(1)$ & $53(1)$ & $24(1)$ & $-18(1)$ & $6(1)$ & $-13(1)$ \\
\hline$C(59)$ & $29(1)$ & $41(1)$ & $23(1)$ & $-15(1)$ & $4(1)$ & $-9(1)$ \\
\hline$C(60)$ & $26(1)$ & $36(1)$ & $23(1)$ & $-13(1)$ & $-1(1)$ & $-8(1)$ \\
\hline$C(61)$ & $27(1)$ & $33(1)$ & $30(1)$ & $-15(1)$ & $-3(1)$ & $-6(1)$ \\
\hline$C(62)$ & $30(1)$ & $62(1)$ & $49(1)$ & $-39(1)$ & $12(1)$ & $-24(1)$ \\
\hline$C(63)$ & $24(2)$ & $22(2)$ & $24(2)$ & $-5(2)$ & $1(2)$ & $-8(2)$ \\
\hline$C(64)$ & $23(2)$ & $28(2)$ & $25(2)$ & $-7(2)$ & $4(2)$ & $-10(2)$ \\
\hline$C(65)$ & $36(2)$ & $31(2)$ & $30(2)$ & $-5(2)$ & $14(2)$ & $-16(2)$ \\
\hline$C(66)$ & $33(2)$ & $52(3)$ & $68(3)$ & $-27(3)$ & $19(2)$ & $-20(2)$ \\
\hline$C\left(63^{\prime}\right)$ & $21(2)$ & $26(2)$ & $22(2)$ & $-9(2)$ & $2(1)$ & $-8(2)$ \\
\hline$C\left(64^{\prime}\right)$ & $24(2)$ & $36(2)$ & $28(2)$ & $-14(2)$ & $7(1)$ & $-16(2)$ \\
\hline$C\left(65^{\prime}\right)$ & $30(2)$ & $51(2)$ & $36(2)$ & $-18(2)$ & $14(2)$ & $-18(2)$ \\
\hline$C\left(66^{\prime}\right)$ & $74(4)$ & $78(4)$ & $73(4)$ & $25(3)$ & $-5(3)$ & $-46(3)$ \\
\hline$C(67)$ & $58(1)$ & $72(2)$ & $42(1)$ & $-38(1)$ & $30(1)$ & $-35(1)$ \\
\hline$C(68)$ & $138(3)$ & $78(2)$ & $32(1)$ & $-19(1)$ & $19(2)$ & $-29(2)$ \\
\hline C(69) & $35(1)$ & $39(1)$ & $40(1)$ & $-22(1)$ & $-3(1)$ & $-9(1)$ \\
\hline
\end{tabular}




\begin{tabular}{|c|c|c|c|c|c|c|}
\hline$C(70)$ & $51(1)$ & $83(2)$ & $33(1)$ & $-21(1)$ & $-11(1)$ & $-5(1)$ \\
\hline$C(71)$ & $63(1)$ & $88(2)$ & $44(1)$ & $-29(1)$ & $14(1)$ & $-58(1)$ \\
\hline$C(72)$ & $52(2)$ & $111(3)$ & 102(3) & $-47(2)$ & $14(2)$ & $-46(2)$ \\
\hline$C(73)$ & $44(2)$ & $36(2)$ & $35(2)$ & $-8(2)$ & $21(2)$ & $-20(2)$ \\
\hline$C(74)$ & $33(2)$ & $81(3)$ & $66(3)$ & $-9(3)$ & $14(2)$ & $-25(2)$ \\
\hline$C\left(73^{\prime}\right)$ & $30(2)$ & $61(3)$ & $42(3)$ & $-30(2)$ & $9(2)$ & $-15(2)$ \\
\hline$C\left(74^{\prime}\right)$ & $65(4)$ & $76(5)$ & $37(3)$ & $-14(3)$ & $17(3)$ & $-31(4)$ \\
\hline$C(75)$ & $37(1)$ & $96(2)$ & $37(1)$ & $-37(1)$ & $18(1)$ & $-27(1)$ \\
\hline$C(76)$ & $66(2)$ & $87(2)$ & $36(1)$ & $-19(1)$ & $13(1)$ & $-32(2)$ \\
\hline$C(77)$ & $43(1)$ & $42(1)$ & $34(1)$ & $-21(1)$ & $0(1)$ & $-12(1)$ \\
\hline $\mathrm{C}(78)$ & $80(2)$ & $70(2)$ & $35(1)$ & $-12(1)$ & $-15(1)$ & $-25(2)$ \\
\hline$C(79)$ & $39(1)$ & $72(2)$ & $67(2)$ & $-48(1)$ & $19(1)$ & $-33(1)$ \\
\hline $\mathrm{C}(80)$ & $36(1)$ & $88(2)$ & 154(3) & $-63(2)$ & $11(2)$ & $-27(1)$ \\
\hline $\mathrm{C}(81)$ & $57(2)$ & $40(2)$ & $52(2)$ & $-25(2)$ & $-12(2)$ & $-5(2)$ \\
\hline $\mathrm{C}(82)$ & $59(3)$ & $42(2)$ & $48(2)$ & $-20(2)$ & $-9(2)$ & $-4(2)$ \\
\hline $\mathrm{C}(83)$ & $56(3)$ & $42(3)$ & $61(3)$ & $-20(2)$ & $-17(3)$ & $-12(2)$ \\
\hline$C(84)$ & $50(2)$ & $49(2)$ & $38(2)$ & $-18(2)$ & $-12(2)$ & $-5(2)$ \\
\hline$C\left(81^{\prime}\right)$ & $34(2)$ & $44(3)$ & $86(4)$ & $22(3)$ & $-10(3)$ & $-19(2)$ \\
\hline$C\left(82^{\prime}\right)$ & $55(4)$ & $29(3)$ & $60(4)$ & $-4(3)$ & $-5(3)$ & $-6(3)$ \\
\hline$C\left(83^{\prime}\right)$ & $32(3)$ & $59(5)$ & $157(12)$ & $23(7)$ & $-17(6)$ & $-7(3)$ \\
\hline$C\left(84^{\prime}\right)$ & $24(2)$ & $44(3)$ & $88(4)$ & $3(3)$ & $-15(2)$ & $-9(2)$ \\
\hline$C(85)$ & $33(2)$ & $54(3)$ & $72(4)$ & $6(3)$ & $-14(2)$ & $-16(2)$ \\
\hline$C(86)$ & $33(2)$ & $74(4)$ & $70(4)$ & $0(3)$ & $-6(2)$ & $-12(3)$ \\
\hline$C(87)$ & $50(3)$ & $39(3)$ & $70(4)$ & $0(3)$ & $25(3)$ & $-13(2)$ \\
\hline $\mathrm{C}(88)$ & $39(3)$ & $48(3)$ & $80(4)$ & $18(3)$ & $-3(3)$ & $-21(2)$ \\
\hline$C\left(85^{\prime}\right)$ & $61(3)$ & $71(3)$ & $22(2)$ & $-9(2)$ & $-5(2)$ & $17(2)$ \\
\hline$C\left(86^{\prime}\right)$ & $95(5)$ & $143(7)$ & $67(4)$ & $-61(4)$ & $-44(4)$ & $66(5)$ \\
\hline$C\left(87^{\prime}\right)$ & $173(9)$ & $60(4)$ & $110(6)$ & $-33(4)$ & $-90(6)$ & $34(5)$ \\
\hline$C\left(88^{\prime}\right)$ & $77(3)$ & $45(2)$ & $52(3)$ & $-23(2)$ & $-25(2)$ & $16(2)$ \\
\hline$C(89)$ & $45(1)$ & $51(1)$ & $36(1)$ & $-19(1)$ & $5(1)$ & $-13(1)$ \\
\hline$C(90)$ & $44(1)$ & $45(1)$ & $79(2)$ & $-10(1)$ & $-3(1)$ & $-9(1)$ \\
\hline$C(91)$ & $42(1)$ & $33(1)$ & $46(1)$ & $-14(1)$ & $3(1)$ & $-4(1)$ \\
\hline$C(92)$ & $45(1)$ & $71(2)$ & $46(1)$ & $-5(1)$ & $1(1)$ & $-24(1)$ \\
\hline$C(93)$ & $39(1)$ & $75(2)$ & $50(1)$ & $-12(1)$ & $3(1)$ & $-16(1)$ \\
\hline$C(94)$ & $45(1)$ & $70(2)$ & $37(1)$ & $-24(1)$ & $0(1)$ & $-1(1)$ \\
\hline$C(95)$ & $47(1)$ & $65(2)$ & 41(1) & $-15(1)$ & $9(1)$ & $-8(1)$ \\
\hline
\end{tabular}


Table S15. Hydrogen coordinates ( x $10^{4}$ ) and isotropic displacement parameters $\left(\AA^{2} \times 10^{3}\right)$ for $\left[(\mathrm{OEP}) \mathrm{Al}(\mathrm{THF})_{2}\right]^{+}\left[\mathrm{Co}(\mathrm{CO})_{4}\right]^{\text {. }}$.

\begin{tabular}{|c|c|c|c|c|}
\hline & $\mathrm{x}$ & $\mathrm{y}$ & $\mathrm{z}$ & $\mathrm{U}(\mathrm{eq})$ \\
\hline $\mathrm{H}(5 \mathrm{~A})$ & 5441 & 2919 & 9511 & 27 \\
\hline $\mathrm{H}(10 \mathrm{~A})$ & 7310 & 5990 & 8547 & 27 \\
\hline $\mathrm{H}(11 \mathrm{~A})$ & 7321 & 5387 & 7848 & 34 \\
\hline $\mathrm{H}(11 \mathrm{~B})$ & 7627 & 4551 & 7780 & 34 \\
\hline $\mathrm{H}(12 \mathrm{~A})$ & 9704 & 5042 & 7722 & 62 \\
\hline $\mathrm{H}(12 \mathrm{~B})$ & 9717 & 4349 & 8247 & 62 \\
\hline $\mathrm{H}(12 \mathrm{C})$ & 9411 & 5187 & 8313 & 62 \\
\hline $\mathrm{H}(13 \mathrm{~A})$ & 6130 & 2913 & 8716 & 38 \\
\hline $\mathrm{H}(13 \mathrm{~B})$ & 6219 & 3489 & 8134 & 38 \\
\hline $\mathrm{H}(14 \mathrm{~A})$ & 8052 & 2518 & 8228 & 66 \\
\hline $\mathrm{H}(14 \mathrm{~B})$ & 8537 & 2705 & 8758 & 66 \\
\hline $\mathrm{H}(14 \mathrm{C})$ & 8630 & 3286 & 8176 & 66 \\
\hline $\mathrm{H}(15 \mathrm{~A})$ & 4020 & 2097 & 9934 & 33 \\
\hline $\mathrm{H}(15 \mathrm{~B})$ & 3290 & 1796 & 10511 & 33 \\
\hline $\mathrm{H}(16 \mathrm{~A})$ & 5365 & 1007 & 10435 & 64 \\
\hline $\mathrm{H}(16 \mathrm{~B})$ & 5475 & 1398 & 10914 & 64 \\
\hline $\mathrm{H}(16 \mathrm{C})$ & 6203 & 1698 & 10336 & 64 \\
\hline $\mathrm{H}(17 \mathrm{~A})$ & 2300 & 2194 & 11243 & 33 \\
\hline $\mathrm{H}(17 \mathrm{~B})$ & 1898 & 2936 & 11451 & 33 \\
\hline $\mathrm{H}(18 \mathrm{~A})$ & 3010 & 1960 & 12142 & 64 \\
\hline $\mathrm{H}(18 \mathrm{~B})$ & 3922 & 2623 & 11967 & 64 \\
\hline $\mathrm{H}(18 \mathrm{C})$ & 4341 & 1885 & 11755 & 64 \\
\hline $\mathrm{H}(19 \mathrm{~A})$ & 3797 & 5173 & 8793 & 36 \\
\hline $\mathrm{H}(19 \mathrm{~B})$ & 3739 & 6012 & 8851 & 36 \\
\hline $\mathrm{H}(20 \mathrm{~A})$ & 1472 & 6276 & 8729 & 44 \\
\hline $\mathrm{H}(20 \mathrm{~B})$ & 1664 & 5557 & 8483 & 44 \\
\hline $\mathrm{H}(21 \mathrm{~A})$ & 331 & 5680 & 9422 & 71 \\
\hline $\mathrm{H}(21 \mathrm{~B})$ & 544 & 4958 & 9182 & 71 \\
\hline $\mathrm{H}(22 \mathrm{~A})$ & 1689 & 4994 & 10069 & 47 \\
\hline $\mathrm{H}(22 \mathrm{~B})$ & 2240 & 4395 & 9726 & 47 \\
\hline
\end{tabular}




\begin{tabular}{|c|c|c|c|c|}
\hline $\mathrm{H}(27 \mathrm{~A})$ & 1512 & 2132 & -666 & 30 \\
\hline $\mathrm{H}(32 \mathrm{~A})$ & -1496 & 713 & 1448 & 30 \\
\hline $\mathrm{H}(33 \mathrm{~A})$ & 2705 & 962 & -2319 & 39 \\
\hline $\mathrm{H}(33 \mathrm{~B})$ & 2091 & 190 & -2168 & 39 \\
\hline $\mathrm{H}(34 \mathrm{~A})$ & 4473 & -30 & -2355 & 80 \\
\hline $\mathrm{H}(34 \mathrm{~B})$ & 4162 & -437 & -1735 & 80 \\
\hline $\mathrm{H}(34 \mathrm{C})$ & 4777 & 334 & -1881 & 80 \\
\hline $\mathrm{H}(35 \mathrm{~A})$ & 3614 & 1786 & -1843 & 40 \\
\hline $\mathrm{H}(35 \mathrm{~B})$ & 3117 & 2158 & -1353 & 40 \\
\hline $\mathrm{H}(36 \mathrm{~A})$ & 2254 & 2951 & -2174 & 72 \\
\hline $\mathrm{H}(36 \mathrm{~B})$ & 998 & 2675 & -1784 & 72 \\
\hline $\mathrm{H}(36 \mathrm{C})$ & 1498 & 2304 & -2275 & 72 \\
\hline $\mathrm{H}(37 \mathrm{~A})$ & 608 & 3127 & 396 & 34 \\
\hline $\mathrm{H}(37 \mathrm{~B})$ & 1411 & 2921 & -124 & 34 \\
\hline $\mathrm{H}(38 \mathrm{~A})$ & -355 & 3931 & -402 & 90 \\
\hline $\mathrm{H}(38 \mathrm{~B})$ & -1470 & 3432 & -80 & 90 \\
\hline $\mathrm{H}(38 \mathrm{C})$ & -663 & 3230 & -599 & 90 \\
\hline $\mathrm{H}(39 \mathrm{~A})$ & -454 & 2582 & 1191 & 38 \\
\hline $\mathrm{H}(39 \mathrm{~B})$ & -572 & 1761 & 1563 & 38 \\
\hline $\mathrm{H}(40 \mathrm{~A})$ & -2659 & 2562 & 1591 & 77 \\
\hline $\mathrm{H}(40 \mathrm{~B})$ & -2899 & 1906 & 1329 & 77 \\
\hline $\mathrm{H}(40 \mathrm{C})$ & -2784 & 2723 & 946 & 77 \\
\hline $\mathrm{H}(41 \mathrm{~A})$ & -1256 & 1201 & -1042 & 61 \\
\hline $\mathrm{H}(41 \mathrm{~B})$ & -2249 & 1539 & -613 & 61 \\
\hline $\mathrm{H}(42 \mathrm{~A})$ & -2982 & 926 & -1423 & 62 \\
\hline $\mathrm{H}(42 \mathrm{~B})$ & -3841 & 1591 & -1194 & 62 \\
\hline $\mathrm{H}(43 \mathrm{~A})$ & -4515 & 264 & -906 & 44 \\
\hline $\mathrm{H}(43 \mathrm{~B})$ & -4750 & 795 & -493 & 44 \\
\hline $\mathrm{H}(44 \mathrm{~A})$ & -3312 & -108 & 42 & 40 \\
\hline $\mathrm{H}(44 \mathrm{~B})$ & -2705 & -453 & -455 & 40 \\
\hline $\mathrm{H}(49 \mathrm{~A})$ & 8260 & 1650 & 3491 & 30 \\
\hline $\mathrm{H}(54 \mathrm{~A})$ & 4459 & 1055 & 5495 & 38 \\
\hline $\mathrm{H}(54 \mathrm{~B})$ & 3708 & 1679 & 5259 & 27 \\
\hline $\mathrm{H}(59 \mathrm{~A})$ & 6993 & 3330 & 6387 & 36 \\
\hline $\mathrm{H}(64 \mathrm{~A})$ & 10919 & 3852 & 4371 & 29 \\
\hline $\mathrm{H}(65 \mathrm{~A})$ & 11614 & 3991 & 3530 & 39 \\
\hline
\end{tabular}




\begin{tabular}{|c|c|c|c|c|}
\hline $\mathrm{H}(65 \mathrm{~B})$ & 11322 & 3811 & 2972 & 39 \\
\hline $\mathrm{H}(66 \mathrm{~A})$ & 13664 & 3494 & 3164 & 72 \\
\hline $\mathrm{H}(66 \mathrm{~B})$ & 13032 & 2756 & 3168 & 72 \\
\hline $\mathrm{H}(66 \mathrm{C})$ & 13320 & 2930 & 3729 & 72 \\
\hline $\mathrm{H}(64 \mathrm{~B})$ & 11462 & 3419 & 4596 & 33 \\
\hline $\mathrm{H}(65 \mathrm{C})$ & 12844 & 3088 & 3935 & 44 \\
\hline $\mathrm{H}(65 \mathrm{D})$ & 12843 & 2797 & 3399 & 44 \\
\hline $\mathrm{H}(66 \mathrm{D})$ & 12912 & 4084 & 3164 & 119 \\
\hline $\mathrm{H}(66 \mathrm{E})$ & 11501 & 4218 & 3496 & 119 \\
\hline $\mathrm{H}(66 \mathrm{~F})$ & 11509 & 3927 & 2960 & 119 \\
\hline $\mathrm{H}(67 \mathrm{~A})$ & 10313 & 1797 & 3019 & 62 \\
\hline $\mathrm{H}(67 \mathrm{~B})$ & 11398 & 2376 & 2866 & 62 \\
\hline $\mathrm{H}(68 \mathrm{~A})$ & 9751 & 2662 & 2192 & 122 \\
\hline $\mathrm{H}(68 \mathrm{~B})$ & 9634 & 3337 & 2476 & 122 \\
\hline $\mathrm{H}(68 \mathrm{C})$ & 8546 & 2760 & 2631 & 122 \\
\hline $\mathrm{H}(69 \mathrm{~A})$ & 5568 & 449 & 3641 & 42 \\
\hline $\mathrm{H}(69 \mathrm{~B})$ & 7065 & 678 & 3476 & 42 \\
\hline $\mathrm{H}(70 \mathrm{~A})$ & 5561 & 1219 & 2750 & 82 \\
\hline $\mathrm{H}(70 \mathrm{~B})$ & 6051 & 1866 & 2963 & 82 \\
\hline $\mathrm{H}(70 \mathrm{C})$ & 4552 & 1639 & 3128 & 82 \\
\hline $\mathrm{H}(71 \mathrm{~A})$ & 4136 & 253 & 4392 & 70 \\
\hline $\mathrm{H}(71 \mathrm{~B})$ & 3728 & 507 & 4939 & 70 \\
\hline $\mathrm{H}(72 \mathrm{~A})$ & 1887 & 887 & 4349 & 122 \\
\hline $\mathrm{H}(72 \mathrm{~B})$ & 2829 & 1350 & 3891 & 122 \\
\hline $\mathrm{H}(72 \mathrm{C})$ & 2425 & 1608 & 4438 & 122 \\
\hline $\mathrm{H}(73 \mathrm{~A})$ & 3778 & 1195 & 6872 & 46 \\
\hline $\mathrm{H}(73 \mathrm{~B})$ & 3593 & 978 & 6319 & 46 \\
\hline $\mathrm{H}(74 \mathrm{~A})$ & 1481 & 1523 & 6611 & 91 \\
\hline $\mathrm{H}(74 \mathrm{~B})$ & 1930 & 2050 & 6045 & 91 \\
\hline $\mathrm{H}(74 \mathrm{C})$ & 2112 & 2262 & 6603 & 91 \\
\hline $\mathrm{H}(73 \mathrm{C})$ & 2581 & 2090 & 6593 & 48 \\
\hline $\mathrm{H}(73 \mathrm{D})$ & 2382 & 1958 & 6010 & 48 \\
\hline $\mathrm{H}(74 \mathrm{D})$ & 2491 & 865 & 6664 & 87 \\
\hline $\mathrm{H}(74 \mathrm{E})$ & 3973 & 966 & 6835 & 87 \\
\hline $\mathrm{H}(74 \mathrm{~F})$ & 3780 & 833 & 6251 & 87 \\
\hline $\mathrm{H}(75 \mathrm{~A})$ & 4829 & 3190 & 6844 & 62 \\
\hline
\end{tabular}




\begin{tabular}{|c|c|c|c|c|}
\hline $\mathrm{H}(75 \mathrm{~B})$ & 3908 & 2533 & 7039 & 62 \\
\hline $\mathrm{H}(76 \mathrm{~A})$ & 5577 & 2406 & 7680 & 91 \\
\hline $\mathrm{H}(76 \mathrm{~B})$ & 6772 & 2363 & 7228 & 91 \\
\hline $\mathrm{H}(76 \mathrm{C})$ & 5856 & 1703 & 7421 & 91 \\
\hline $\mathrm{H}(77 \mathrm{~A})$ & 8138 & 4343 & 6369 & 44 \\
\hline $\mathrm{H}(77 \mathrm{~B})$ & 9626 & 4584 & 6204 & 44 \\
\hline $\mathrm{H}(78 \mathrm{~A})$ & 9603 & 3876 & 7114 & 90 \\
\hline $\mathrm{H}(78 \mathrm{~B})$ & 10664 & 3434 & 6765 & 90 \\
\hline $\mathrm{H}(78 \mathrm{C})$ & 9174 & 3191 & 6929 & 90 \\
\hline $\mathrm{H}(79 \mathrm{~A})$ & 11121 & 4722 & 5481 & 62 \\
\hline $\mathrm{H}(79 \mathrm{~B})$ & 11510 & 4471 & 4931 & 62 \\
\hline $\mathrm{H}(80 \mathrm{~A})$ & 13398 & 4108 & 5506 & 127 \\
\hline $\mathrm{H}(80 \mathrm{~B})$ & 12879 & 3379 & 5424 & 127 \\
\hline $\mathrm{H}(80 \mathrm{C})$ & 12495 & 3633 & 5973 & 127 \\
\hline $\mathrm{H}(81 \mathrm{~A})$ & 5697 & 4109 & 5047 & 56 \\
\hline $\mathrm{H}(81 \mathrm{~B})$ & 6973 & 4392 & 4672 & 56 \\
\hline $\mathrm{H}(82 \mathrm{~A})$ & 5618 & 5059 & 4000 & 58 \\
\hline $\mathrm{H}(82 \mathrm{~B})$ & 4384 & 4968 & 4449 & 58 \\
\hline $\mathrm{H}(83 \mathrm{~A})$ & 3814 & 3948 & 4220 & 61 \\
\hline $\mathrm{H}(83 \mathrm{~B})$ & 4255 & 4437 & 3631 & 61 \\
\hline $\mathrm{H}(84 \mathrm{~A})$ & 6465 & 3791 & 3691 & 53 \\
\hline $\mathrm{H}(84 \mathrm{~B})$ & 5520 & 3143 & 3912 & 53 \\
\hline $\mathrm{H}(81 \mathrm{C})$ & 7490 & 3954 & 3955 & 72 \\
\hline $\mathrm{H}(81 \mathrm{D})$ & 7681 & 4114 & 4536 & 72 \\
\hline $\mathrm{H}(82 \mathrm{C})$ & 5684 & 4823 & 4505 & 59 \\
\hline $\mathrm{H}(82 \mathrm{D})$ & 5871 & 4925 & 3861 & 59 \\
\hline $\mathrm{H}(83 \mathrm{C})$ & 4318 & 4241 & 3848 & 110 \\
\hline $\mathrm{H}(83 \mathrm{D})$ & 3861 & 4356 & 4440 & 110 \\
\hline $\mathrm{H}(84 \mathrm{C})$ & 4637 & 3161 & 4819 & 66 \\
\hline $\mathrm{H}(84 \mathrm{D})$ & 5043 & 3039 & 4220 & 66 \\
\hline $\mathrm{H}(85 \mathrm{~A})$ & 10284 & 1842 & 5717 & 67 \\
\hline $\mathrm{H}(85 \mathrm{~B})$ & 10629 & 1848 & 5087 & 67 \\
\hline $\mathrm{H}(86 \mathrm{~A})$ & 11034 & 544 & 5282 & 74 \\
\hline $\mathrm{H}(86 \mathrm{~B})$ & 11513 & 633 & 5852 & 74 \\
\hline $\mathrm{H}(87 \mathrm{~A})$ & 9537 & 376 & 6273 & 68 \\
\hline $\mathrm{H}(87 \mathrm{~B})$ & 9503 & -76 & 5820 & 68 \\
\hline
\end{tabular}




\begin{tabular}{lrrrr}
$\mathrm{H}(88 \mathrm{~A})$ & 7773 & 742 & 5398 & 73 \\
$\mathrm{H}(88 \mathrm{~B})$ & 7610 & 1078 & 5925 & 73 \\
$\mathrm{H}(85 \mathrm{C})$ & 9415 & 1670 & 6106 & 65 \\
$\mathrm{H}(85 \mathrm{D})$ & 8353 & 1079 & 6182 & 65 \\
$\mathrm{H}(86 \mathrm{C})$ & 11229 & 835 & 5990 & 123 \\
$\mathrm{H}(86 \mathrm{D})$ & 10300 & 277 & 6388 & 123 \\
$\mathrm{H}(87 \mathrm{C})$ & 9798 & -161 & 5769 & 137 \\
$\mathrm{H}(87 \mathrm{D})$ & 11146 & 153 & 5471 & 137 \\
$\mathrm{H}(88 \mathrm{C})$ & 8570 & 579 & 5121 & 69 \\
$\mathrm{H}(88 \mathrm{D})$ & 9909 & 926 & 4840 & 69 \\
& & & & \\
\hline
\end{tabular}

